\title{
NORTHWESTERN UNIVERSITY
}

\author{
Study of $B_{s}^{0}$ Mixing at the D-Zero Detector at Fermilab Using the \\ Semi-leptonic Decay $B_{s}^{0} \rightarrow D_{s}^{-} \mu^{+} \nu X$
}

\section{A DISSERTATION}

SUBMITTED TO THE GRADUATE SCHOOL IN PARTIAL FULFILLMENT OF THE REQUIREMENTS

for the degree

DOCTOR OF PHILOSOPHY

Field of Physics

By

Meghan S. Anzelc

EVANSTON, ILLINOIS

June 2008 
(C) Copyright by Meghan S. Anzelc 2008

All Rights Reserved 


\section{ABSTRACT}

Study of $B_{s}^{0}$ Mixing at the D-Zero Detector at Fermilab Using the Semi-leptonic Decay $B_{s}^{0} \rightarrow D_{s}^{-} \mu^{+} \nu X$

Meghan S. Anzelc

$B_{s}^{0}$ mixing studies provide a precision test of Charge-Parity violation in the Standard Model. A measurement of $\Delta m_{s}$ constrains elements of the CKM quark rotation matrix [1], providing a probe of Standard Model Charge-Parity violation.

This thesis describes a study of $B_{s}^{0}$ mixing in the semileptonic decay $B_{s}^{0} \rightarrow D_{s}^{-} \mu^{+} \nu X$, where $D_{s}^{-} \rightarrow \phi \pi^{-}$, using data collected at the D-Zero detector at Fermi National Accelerator in Batavia, Illinois. Approximately $2.8 \mathrm{fb}^{-1}$ of data collected between April 2002 and August 2007 was used, covering the entirety of the Tevatron's RunIIa (April 2002 to March 2006) and part of RunIIb (March 2006 - August 2007).

Taggers using both opposite-side and same-side information were used to obtain the flavor information of the $B_{s}^{0}$ meson at production. The charge of the muon in the decay $B_{s}^{0} \rightarrow D_{s}^{-} \mu^{+} \nu X$ was used to determine the flavor of the $B_{s}^{0}$ at decay. The $B_{d}^{0}$ mixing 
frequency, $\Delta m_{d}$, was measured to verify the analysis procedure. A log-likelihood calculation was performed, and a measurement of $\Delta m_{s}$ was obtained. The final result was $\Delta m_{s}=18.86 \pm 0.80$ (stat.) \pm 0.37 (sys.) with a significance of $2.6 \sigma$. 


\section{Acknowledgements}

I could not have gotten to this point without the help of a number of people, who all deserve my thanks. First, a big thanks to my advisor Dave Buchholz, for all of his guidance throughout my time at Northwestern, and for always supporting me, in both words and deeds.

Thanks to Sergey Burdin, for entrusting his analysis to me and for all of our discussions of the details of the analysis. Thanks also to Brendan Casey, for his continual mentoring and for always being in my corner. I also want to thank Heidi Schellman and André de Gouvêa for serving on both my candidacy and defense committees, and for all of their assistance and help. Additionally, I am grateful to many of the members of the B-physics group for their assistance throughout my time at Fermilab.

I would also like to thank my undergraduate professors, especially Dr. G., Dr. Mallow, and a special thanks to Dr. Ramsey. The innumerable hours spent discussing physics and the care and support they gave me are a large part of my success in graduate school.

I could not have had a better group of friends in graduate school. Thanks to Jon, Andrew, Jeff and Johannes, for the long days studying and the many lunches together; amazingly, what I remember most is how much we laughed. Special thanks to Jennifer and Kendall; I can't imagine grad school without you both. Thanks also to Tim, Derek SungWoo and Sahal; work is always easier when you're surrounded by good people, and I've had great company. 
Thanks to my friends, who have always been there for me - you know who you are. Special thanks to Sarah, for reminding me there are more difficult things than grad school, and my love and thanks to Megan and my sister Aislinn, for everything. And I am lucky to have the greatest in-laws, and am especially grateful for how understanding they have been of this whole process.

To my parents, "thanks" is too small a word. I would not be here if they hadn't spent so much of their lives dedicated to my education. Thank you. If anyone asks if a home-schooled kid can get into college, you have my permission to hand them a copy of this.

Finally, thanks to my husband Nick. I got the better end of the deal. 


\section{Table of Contents}

ABSTRACT

Acknowledgements

List of Tables

List of Figures

Chapter 1. Introduction

1.1. The Standard Model

1.2. Overview of This Analysis

Chapter 2. Experimental Apparatus - Tevatron and the D-Zero Detector

2.1. The Tevatron at Fermilab

33

2.2. The D-Zero Detector

33

Chapter 3. Analvsis

3.1. Historical Perspective

47

3.2. Particle Reconstruction and Event Selection

3.3. Flavor Tagoing

3.4. Mass Fitting Procedure

68

3.5. Unbinned Likelihood Fit Method

70

3.6. Inputs to the Fit

81 


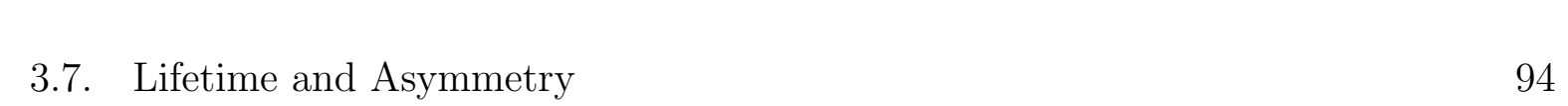

3.8. Fit for $\Delta m_{\text {d }} \quad 95$

\begin{tabular}{|l|l|}
\hline Chapter 4. Results & 99
\end{tabular}

4.1. Svstematic Uncertainties 101

4.2. Cross-checks 113

4.3. Determination of the Significance of the Result 119

$\begin{array}{ll}\text { 4.4. Conclusions } & 125\end{array}$

4.5. Discussion 125

\begin{tabular}{ll}
\hline References & 127
\end{tabular}

Appendix A. Branching Rates 132

Appendix B. VPDL pdf Plots 134

Appendix C. List of IP-Biased Triggers in RunIIa and RunIIb Data 137

$\begin{array}{ll}\text { C.1. RunIIa } & 137\end{array}$

$\begin{array}{ll}\text { C.2. RunIIb } & 138\end{array}$ 


\section{List of Tables}

\begin{tabular}{|c|c|c|}
\hline 3.1 & Mixing in the Standard Model & 48 \\
\hline 3.2 & $\operatorname{Mass}\left(\mu^{+} D_{0}^{-}\right)$Bins & 84 \\
\hline 3.3 & RunIIa Sample Composition & 88 \\
\hline 3.4 & RunIIb Sample Composition & 89 \\
\hline 3.5 & Lifetime fit parameters for RunIIa and RunIIb & 94 \\
\hline 4.1 & Svstematics Summarv & 112 \\
\hline 4.2 & RunIIa Svstematic Uncertainties & 114 \\
\hline 4.3 & RunIIb Systematic Uncertainties & 115 \\
\hline 4.4 & Combined RunIIa and RunIIb Svstematic Unc & 116 \\
\hline
\end{tabular}




\section{List of Figures}

\begin{tabular}{|c|c|}
\hline 1.1 & The Standard Model of fundamental particles \\
\hline 1.2 & $B_{0}^{0}$ mixing Fevnman box diagrams \\
\hline 1.3 & The Unitarity Triangle in the Wolfenstein parameterization \\
\hline 1.4 & Current Understanding of the Unitarity Triangle \\
\hline 1.5 & Illustration of a $B_{0}^{0} \rightarrow \mu \phi \pi$ event \\
\hline 2.1 & The D-Zero detector \\
\hline 2.2 & The RunII D-Zero tracking svstems \\
\hline 2.3 & The RunII D-Zero Silicon Microstrip Tracker \\
\hline 2.4 & The RunII D-Zero Silicon Microstrip Tracken \\
\hline 2.5 & D-Zero Central Fiber Tracker Laver \\
\hline 2.6 & D-Zero Preshower Detectors \\
\hline 2.7 & D-Zero Preshower Detectors \\
\hline 2.8 & D-Zero Muon Svstem \\
\hline 3.1 & The Unitarity Triangle Limits as of 2005 \\
\hline 3.2 & The Unitarity Triangle Limits After D-Zero's Upper-Limit \\
\hline 3.3 & The Current Unitarity Triangle Limits \\
\hline
\end{tabular}


$3.4 \quad$ Illustration of a tvpical $B_{0}^{0}$ event $\quad 55$

$\begin{array}{lll}3.5 & \text { Flavor asvmmetries using OST } & 58\end{array}$

$3.6 \quad$ The OST Calibration Curve $\quad 59$

\begin{tabular}{|ll}
3.7 & Same-Side Fragmentation Illustration
\end{tabular}

$\begin{array}{|ll|}3.8 & \text { Dilution versus }|d| \text { for the combined SST }\end{array}$

$3.9 \quad$ The SST Calibration Curves $\quad 63$

\begin{tabular}{|ll}
$3.10 \quad$ Flavor Tagging Flowchart & 64
\end{tabular}

$3.11 \quad$ Dilution versus $|d|$ for the Combined Tag in Md 64

$3.12 \quad$ Dilution versus $|d|$ for the Combined Tag in MC and Data 65

3.13 The Combined Tag Calibration Curve 65

$3.14 \quad$ Same-Side Fragmentation Illustration $\quad 66$

\begin{tabular}{|ll}
3.15 & The $B^{+}$Calibration Curve
\end{tabular}

$3.16 \quad$ RunIIa Total $B_{\text {S Sample }}^{0} \quad 69$

$3.17 \quad$ RunIIa Tagged $B_{0}^{0}$ Sample $\quad 69$

$3.18 \quad$ RunIIb Total $B_{0}^{0}$ Sample $\quad 69$

$3.19 \quad$ RunIIb Tagged $B^{0}$ Sample $\quad 69$

\begin{tabular}{lll}
\hline $3.20 \quad$ RunIIa VPDL $p d t$ & 72
\end{tabular}

\begin{tabular}{|ll}
\hline $3.21 \quad$ RunIIb VPDL $p d t$ & 72
\end{tabular}

$3.22 \quad$ RunIIa and RunIIb VPDL $p d]$

$3.23 \quad$ RunIIa Dilution $p d$ J

\begin{tabular}{|ll}
\hline 3.24 & RunIIb Dilution $p d$
\end{tabular} 


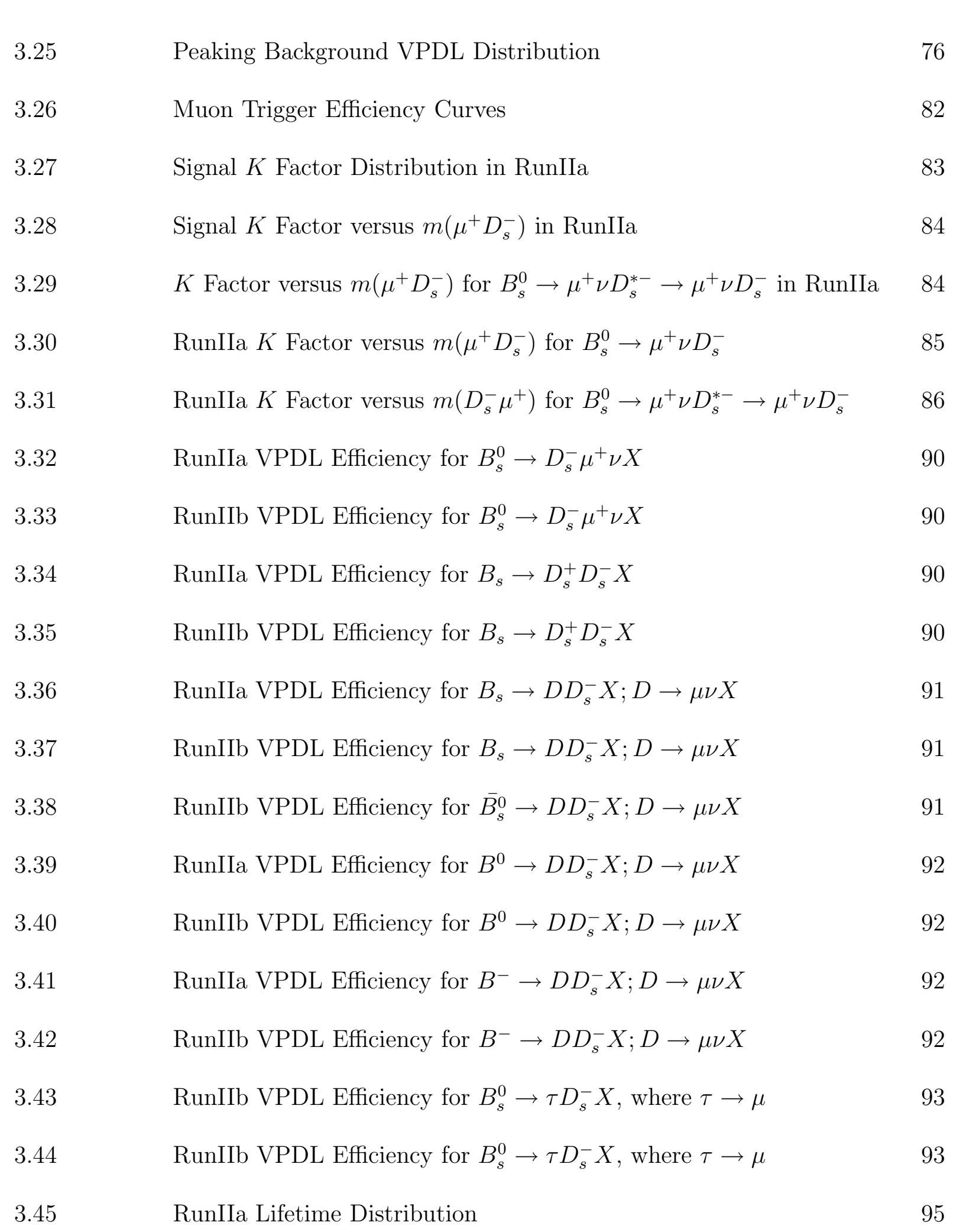


$3.46 \quad$ RunIIb Lifetime Distribution 95

$4.1 \quad$ RunIIa Amplitude Scan. Statistical Only 100

$4.2 \quad$ RunIIb Amplitude Scan. Statistical Only 101

$4.3 \quad$ RunIIa Amplitude Scan 102

$4.4 \quad$ RunIIb Amplitude Scan 103

$4.5 \quad$ Combined RunIIa and RunIIb Amplitude Scan. Statistical Only 104

$4.6 \quad$ Combined RunIIa and RunIIb Amplitude Scan 105

$4.7 \quad$ Combined RunIIa and RunIIb Log-likelihood. Statistical Onlv 106

$4.8 \quad B_{0}^{0}$ Dilution Systematic Curves 106

\begin{tabular}{lll}
\hline 4.9 & $B^{+}$Dilution Svstematic Curves & 107
\end{tabular}

$4.10 \quad$ RunIIa and RunIIb $J / \psi \rightarrow \mu^{+} \mu^{-}$Pull Distributions 108

$4.11 \quad$ Likelihoods Using Default and Flat Efficiencv Curves 110

$4.12 \quad B_{d}^{0}$ Amplitude in RunIIa 117

$4.13 \quad$ Detail of $B_{d}^{0}$ Amplitude in RunIIa 117

\begin{tabular}{|lll}
\hline 4.14 & $B_{d}^{0}$ Amplitude in RunIIb & 117
\end{tabular}

$4.15 \quad$ Detail of $B_{d}^{0}$ Amplitude in RunIIb 117

$4.16 \quad$ RunIIa Mass Plot of IP Biased Events 118

$4.17 \quad$ RunIIb Mass Plot of IP Biased Events 118

$4.18 \quad$ RunIIa Amplitude Scan Excluding IP Biased Events 119

$4.19 \quad$ RunIIb Amplitude Scan Excluding IP Biased Events 119 


\begin{tabular}{|c|c|}
\hline 4.20 & RunIIa Random Tag Amplitude \\
\hline 4.21 & RunIIb Random Tag Amplitude \\
\hline 4.22 & RunIIa+RunIIb Ensemble Test Results \\
\hline 4.23 & Log-likelihood for RunIIa and RunIIb. Statistical+Svstematid \\
\hline B.1 & RunIIa and RunIIb VPDL pdf. Background and Signal Overlaid \\
\hline B.2 & RunIIa VPDL $p d f$. With and Without $S F$ s Applied \\
\hline B. 3 & RunIIb VPDL $p d f$. With and Without $S F$ s Applied \\
\hline B.4 & RunIIa VPDL $p d f$. Without SFs \\
\hline B.5 & RunIIb VPDL $p d f$. Without $S F \mathrm{~s}$ \\
\hline
\end{tabular}




\section{CHAPTER 1}

\section{Introduction}

Physicists believe the Big Bang produced a slightly greater amount of matter than antimatter, allowing the universe to develop into the matter universe we see today. Physicists need to determine whether our current conceptual framework, called the Standard Model (SM), explains this asymmetry of matter and anti-matter in the early universe or if we must look to new theories to explain the asymmetry.

A probe of this asymmetry is provided by studies of $B_{s}^{0}$ mixing. Currently, the only studies of $B_{s}^{0}$ mixing have come from the Tevatron at Fermi National Accelerator Laboratory in Batavia, Illinois. This thesis describes $B_{s}^{0}$ mixing studies in one decay channel that were performed at the D-Zero detector, one of two detectors at the Tevatron.

\subsection{The Standard Model}

Our current understanding of fundamental particles and their interactions is called the Standard Model [2]. The fundamental particles and forces of the Standard Model are shown in Figure 1.1. The Standard Model consists of six quarks, of which the up and down quarks make up most of the everyday matter around us. The $B_{s}^{0}$ meson is composed

of a strange quark, the $s$ quark, and an anti-bottom quark $\bar{b}$. Its anti-partner, a $\bar{B}_{s}^{0}$, is composed of an $\bar{s}$ and a $b$ quark.

In addition to the six quarks there are six leptons. These are the electron, muon, and tau, along with their corresponding neutrinos: $\nu_{e}, \nu_{\mu}$, and $\nu_{\tau}$. The right column of 


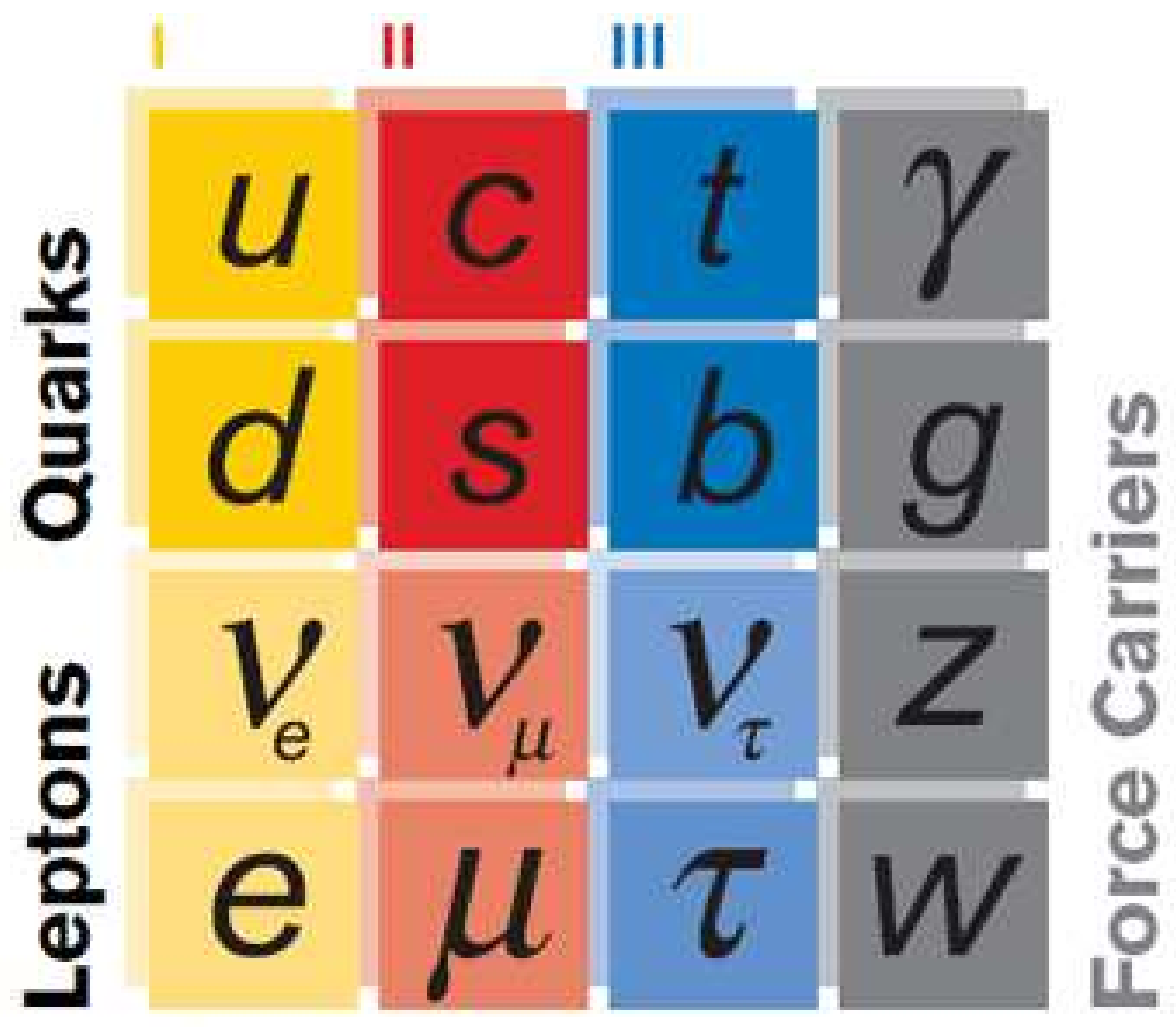

\section{Three Generations of Matter}

Figure 1.1. The Standard Model, containing sixteen fundamental particles, separated into quark and lepton categories, as well as the four force carriers. The generations are labeled as I, II, and III at the top of the figure, with I being the lightest and III the heaviest category.

Figure 1.1 shows the force carriers for the four fundamental forces. The four fundamental forces are gravity, the electromagnetic force, the weak force, and the strong force. While gravity is not described by the SM, this force is known to be much weaker than the other three fundamental forces, about 25 orders of magnitude weaker than the weak force. Gravity is believed to be mediated by the graviton, but the graviton has not yet been observed. The electromagnetic force is mediated by the photon, the weak force is mediated by the charged $W^{ \pm}$bosons and the neutral $Z$ boson, and the strong force is mediated by 
the gluon. $B_{s}^{0}$ mixing occurs via the weak force, through an exchange of two $W$ bosons, shown in Figure 1.2.

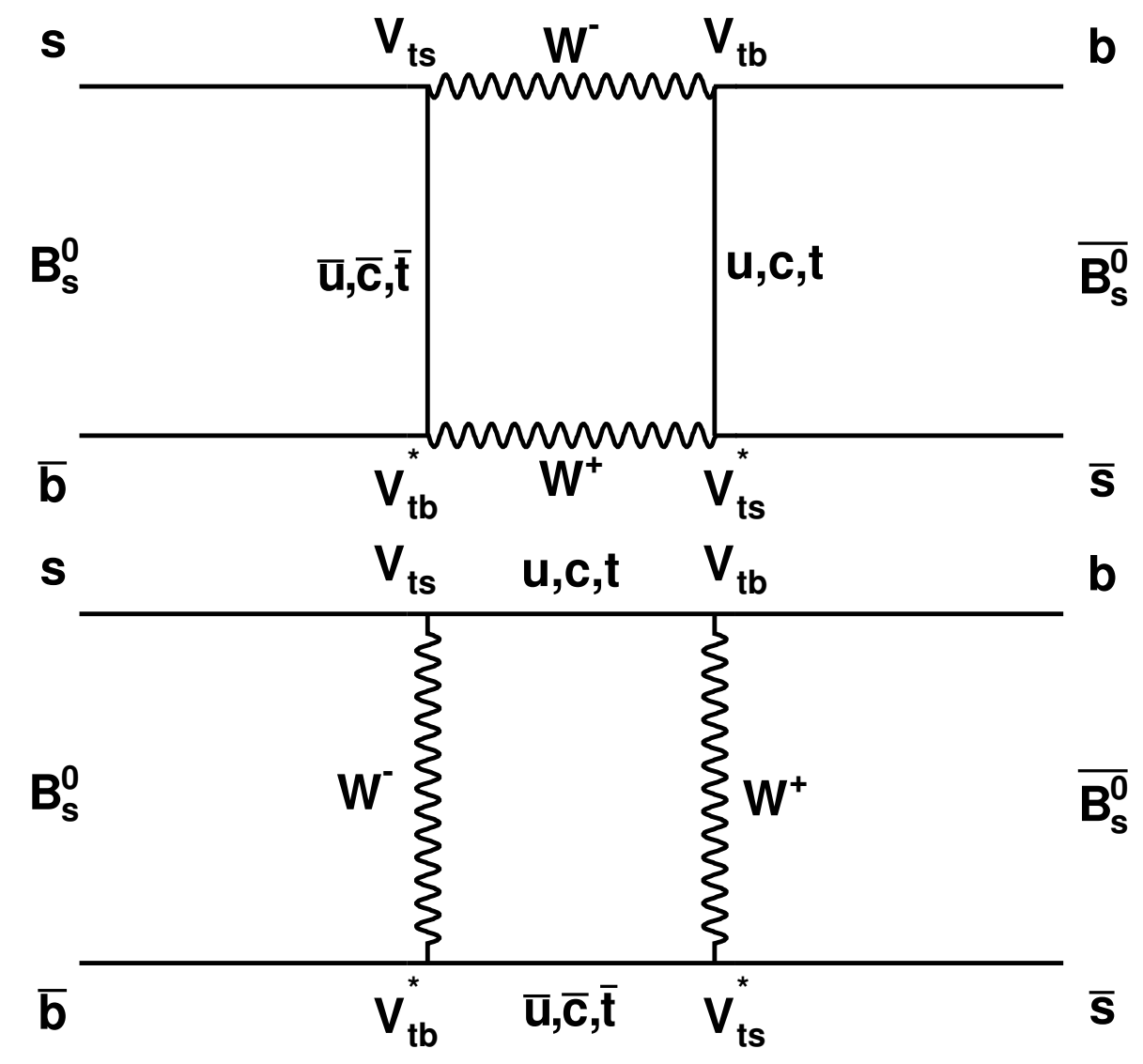

Figure 1.2. The two Feynman box diagrams that give rise to $B_{s}^{0}$ mixing in the Standard Model. The relevant CKM matrix elements, discussed in Section 1.1 .2 are shown at the interaction points.

The three standard symmetries discussed in the Standard Model are: charge, parity, and time. If an interaction is symmetric in charge, then the interaction is the same when you reverse the particles with their antiparticles. If an interaction is symmetric in parity, then it looks the same when viewed mirror-image and upside-down. Similarly, if an interaction is symmetric in time, the interaction is the same going backwards in time as it 
is going forwards in time. These symmetries can also be discussed in combination, such as Charge-Parity (CP) conservation or violation. The Standard Model allows a certain amount of $\mathrm{CP}$ violation that accounts for some of the matter/antimatter asymmetry. The interesting question is whether the amount of $\mathrm{CP}$ violation in the Standard Model accurately describes the matter/anti-matter asymmetry of the early universe. Studying $B_{s}^{0}$ mixing gives information about the amount of $\mathrm{CP}$ violation in the universe, which can then be compared to the amount predicted by the SM. How $B_{s}^{0}$ mixing studies relates to $\mathrm{CP}$ violation will be described later in this chapter.

\subsubsection{Formalism of Mixing}

The Standard Model quarks can be described by a doublet in the mass eigenstate representation, as:

$$
\left(\begin{array}{l}
u \\
d
\end{array}\right)
$$

In 1963, motivated by experimental Kaon decay rates, Cabibbo proposed that the weak eigenstates could be represented by the mass eigenstate doublet and a quark rotation angle, $\theta_{c}$ [3. The doublet above can then be expressed as:

$$
\left(\begin{array}{l}
u \\
d^{\prime}
\end{array}\right)=\left(\begin{array}{c}
u \\
d \cos \theta_{C}+s \sin \theta_{C}
\end{array}\right)
$$

where $d^{\prime}$ is the weak eigenstate of the down quark, a combination of the mass eigenstates of the down and strange quarks and the Cabibbo angle. 
When Glashow, Illiopoulus, and Maiani proposed the charm quark in 1970 [4], the formalism introduced by Cabibbo was expanded to two doublets and had a 2x2 quark rotation matrix, as shown in Equation 1.4. The first of the two doublets is shown above in Equation 1.2 and the second in Equation 1.3. This mechanism, known as the "GIM" mechanism, completed the picture for the three known quarks and newly proposed charm quark.

$$
\begin{gathered}
\left(\begin{array}{c}
c \\
s^{\prime}
\end{array}\right)=\left(\begin{array}{c}
c \\
-d \sin \theta_{C}+s \cos \theta_{C}
\end{array}\right) \\
\left(\begin{array}{c}
d^{\prime} \\
s^{\prime}
\end{array}\right)=\left(\begin{array}{cc}
\cos \theta_{C} & \sin \theta_{C} \\
-\sin \theta_{C} & \cos \theta_{C}
\end{array}\right)\left(\begin{array}{l}
d \\
s
\end{array}\right)
\end{gathered}
$$

Later, when the Standard Model was expanded [1] to include the bottom and top quarks, the rotation matrix was modified again to a 3x3 rotation matrix. This was done by Kobayashi and Maskawa and is known as the CKM matrix.

\subsubsection{The CKM Matrix}

The Cabibbo-Kobayashi-Maskawa, or CKM, matrix is a 3x3 unitary matrix which describes the flavor-change of quarks in the Standard Model. It is described by three real parameters and one complex phase. The three real parameters are analogous to the Euler 
angles, while CP violation in the Standard Model arises from the complex phase. There are a variety of ways to parameterize the CKM matrix. The most general form is:

$$
\left(\begin{array}{ccc}
V_{u d} & V_{u s} & V_{u b} \\
V_{c d} & V_{c s} & V_{c b} \\
V_{t d} & V_{t s} & V_{t b}
\end{array}\right)
$$

where each element $V_{q q^{\prime}}$ describes the coupling for the given transition. The larger the magnitude of $V_{q q^{\prime}}$, the greater the strength of the flavor-changing transition.

The CKM matrix can also be described by the weak mixing angle, $\theta_{C}$, and the complex phase $\delta$ as:

$$
\left(\begin{array}{ccc}
c_{12} c_{13} & s_{12} c_{13} & s_{13} e^{-i \delta} \\
-s_{12} c_{23}-c_{12} s_{23} s_{13} e^{i \delta} & c_{12} c_{23}-s_{12} s_{23} s_{13} e^{i \delta} & s_{23} c_{13} \\
s_{12} s_{23}-c_{12} c_{23} s_{13} e^{i \delta} & -c_{12} s_{23}-s_{12} c_{23} s_{13} e^{i \delta} & c_{23} c_{13}
\end{array}\right),
$$

where "s" stands for $\sin \theta$ and "c" for $\cos \theta$.

While there are a number of parameterizations of the CKM matrix, such as the one above, the standard parameterization used was done by Wolfenstein [5]. This uses four parameters: $A, \lambda, \rho$, and $\eta$, where $\eta$ is the complex parameter. This parameterization of the CKM matrix is given by:

$$
\left(\begin{array}{ccc}
1-\frac{1}{2} \lambda^{2} & \lambda & A \lambda^{3}(\rho-i \eta) \\
-\lambda & 1-\frac{1}{2} \lambda^{2} & A \lambda^{2} \\
A \lambda^{3}(1-\rho-i \eta) & -A \lambda^{2} & 1
\end{array}\right) .
$$


The most recent [6] PDG values and uncertainties for the CKM matrix elements, assuming unitarity, are:

$$
\left(\begin{array}{ccc}
0.97383_{-0.00023}^{+0.00024} & 0.2272 \pm 0.0010 & (3.96 \pm 0.09) \times 10^{-3} \\
0.2271 \pm 0.0010 & 0.97296 \pm 0.00024 & \left(42.21_{-0.80}^{+0.10}\right) \times 10^{-3} \\
\left(8.14_{-0.64}^{+0.32}\right) \times 10^{-3} & \left(41.61_{-0.78}^{+0.12}\right) \times 10^{-3} & 0.999100_{-0.000004}^{+0.000034}
\end{array}\right)
$$

From this it can be seen that the CKM matrix element $\left|V_{t d}\right|$, in the lower-left corner, has the largest uncertainty of the elements, at over $4 \%$.

\subsubsection{The Unitarity Triangle}

By definition, the unitarity of the CKM matrix means all of the rows and columns are orthogonal. This allows one to construct six different triangles from the orthogonal combinations of rows and columns. The six combinations are:

$$
\begin{aligned}
& V_{u d} V_{u s}^{*}+V_{c d} V_{c s}^{*}+V_{t d} V_{t s}^{*}=0, \\
& V_{u s} V_{u b}^{*}+V_{c s} V_{c b}^{*}+V_{t s} V_{t b}^{*}=0, \\
& V_{u d} V_{u b}^{*}+V_{c d} V_{c b}^{*}+V_{t d} V_{t b}^{*}=0, \\
& V_{u d} V_{c d}^{*}+V_{u s} V_{c s}^{*}+V_{u b} V_{c b}^{*}=0, \\
& V_{c d} V_{t d}^{*}+V_{c s} V_{t s}^{*}+V_{c b} V_{t b}^{*}=0, \\
& V_{u d} V_{t d}^{*}+V_{u s} V_{t s}^{*}+V_{u b} V_{t b}^{*}=0 .
\end{aligned}
$$

Due to the magnitude of the nine CKM elements, four of the six triangles have two long sides of nearly equal length and one very short side resulting in "squished" triangles. 
It is therefore difficult to adequately probe the CP-volating parameters of the "squished" triangles. The other two triangles, from equations 1.11 and 1.14 have more even proportions. In the Wolfenstein parameterization, these two triangles are equivalent up to order $\lambda^{3}$, reducing to one equation:

$$
((\rho+i \eta)-1+(1-\rho-i \eta)) A \lambda^{3}=0
$$

This translates into one useful triangle and is therefore usually the only one used by physicists. It can be rescaled such that one side of the triangle aligns with the real axis, where

$$
\bar{\rho}=\rho\left(1-\lambda^{2} / 2\right)
$$

and

$$
\bar{\eta}=\eta\left(1-\lambda^{2} / 2\right)
$$

This places one corner at $(0,0)$, one at $(0,1)$, and the apex at $(\bar{\rho}, \bar{\eta})$ in the complex plane. The angles of the triangle are then defined as:

$$
\alpha=\tan ^{-1}\left(\frac{\bar{\eta}}{\bar{\eta}^{2}+\bar{\rho}(\bar{\rho}-1)}\right), \beta=\tan ^{-1}\left(\frac{\bar{\eta}}{1-\bar{\rho}}\right), \gamma=\tan ^{-1}\left(\frac{\bar{\eta}}{\bar{\rho}}\right)
$$

and the two sides of the triangle are:

$$
R_{u}=\overline{\alpha \beta}=\frac{1-\lambda^{2} / 2}{\lambda}\left|\frac{V_{u b}}{V_{c b}}\right|,
$$


and

$$
R_{t}=\overline{\beta \gamma}=\frac{1}{\lambda}\left|\frac{V_{t d}}{V_{c b}}\right| .
$$

This is known as the "Unitarity Triangle" [7] and is shown in Figure $1.3 \quad B_{s}^{0}$ and $B_{d}^{0}$ mixing studies reduce the uncertainty on the length of side $R_{t}$.

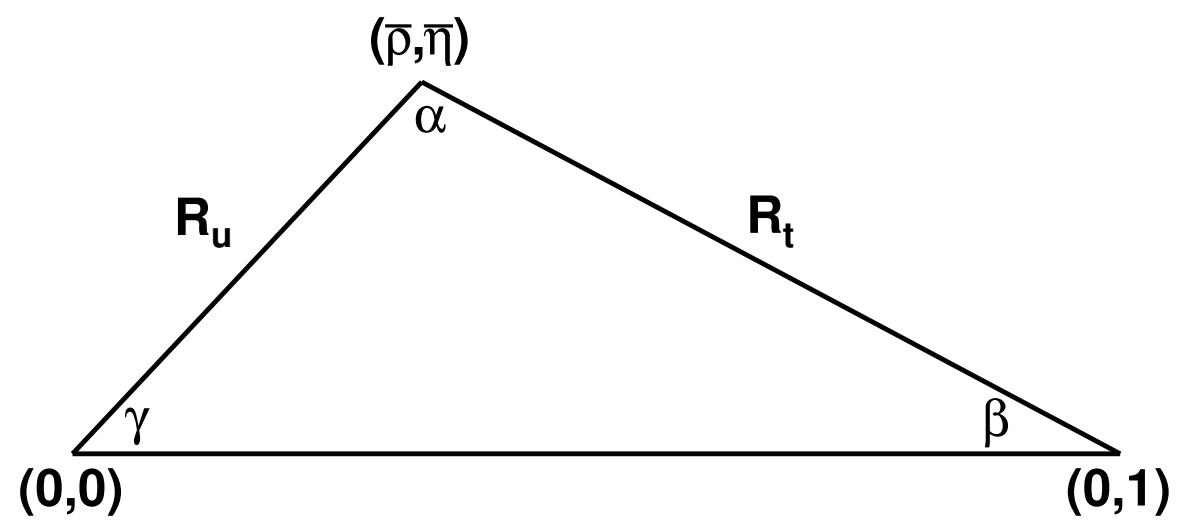

Figure 1.3. The Unitarity Triangle in the Wolfenstein parameterization.

The current understanding of the Unitarity Triangle is shown in Figure 1.4 produced by the CKMfitter group $[8$. The blue wedge marks the $\sin (2 \beta)$ measurements done by the B-factories $\left[\mathbf{9}\right.$, and the green $\epsilon_{K}$ bands are CP-violation measurements in kaons $[\mathbf{6}$. The green circle centered at $(0,0)$ is $\left|V_{u b}\right|$ constraints from CLEO, LEP, CDF (the Collider Detector at Fermilab, the other Tevatron experiment), and the B-factories [9]. Additionally, direct constraints on $\alpha$ and $\gamma$ have been provided by the B-factories. The yellow circle gives the constraints on the unitarity triangle from measurements of the $B_{d}^{0}$ oscillation frequency, $\Delta m_{d}[\mathbf{9}$, while the smaller orange circular band indicates the constraints from both the $\Delta m_{d}$ and $\Delta m_{s}$ studies [10]. The small yellow oval outlined in red at the apex of the triangle shows the current uncertainty on the length of the side of the unitarity 
triangle $R_{t}$ (see Equation 1.19). Further lower-limits on $\Delta m_{s}$ will constrain the orange band from the left, while additional constraints on the upper limit of $\Delta m_{s}$ will push the orange band inwards from the right, thus decreasing the uncertainty on the length of $R_{t}$.

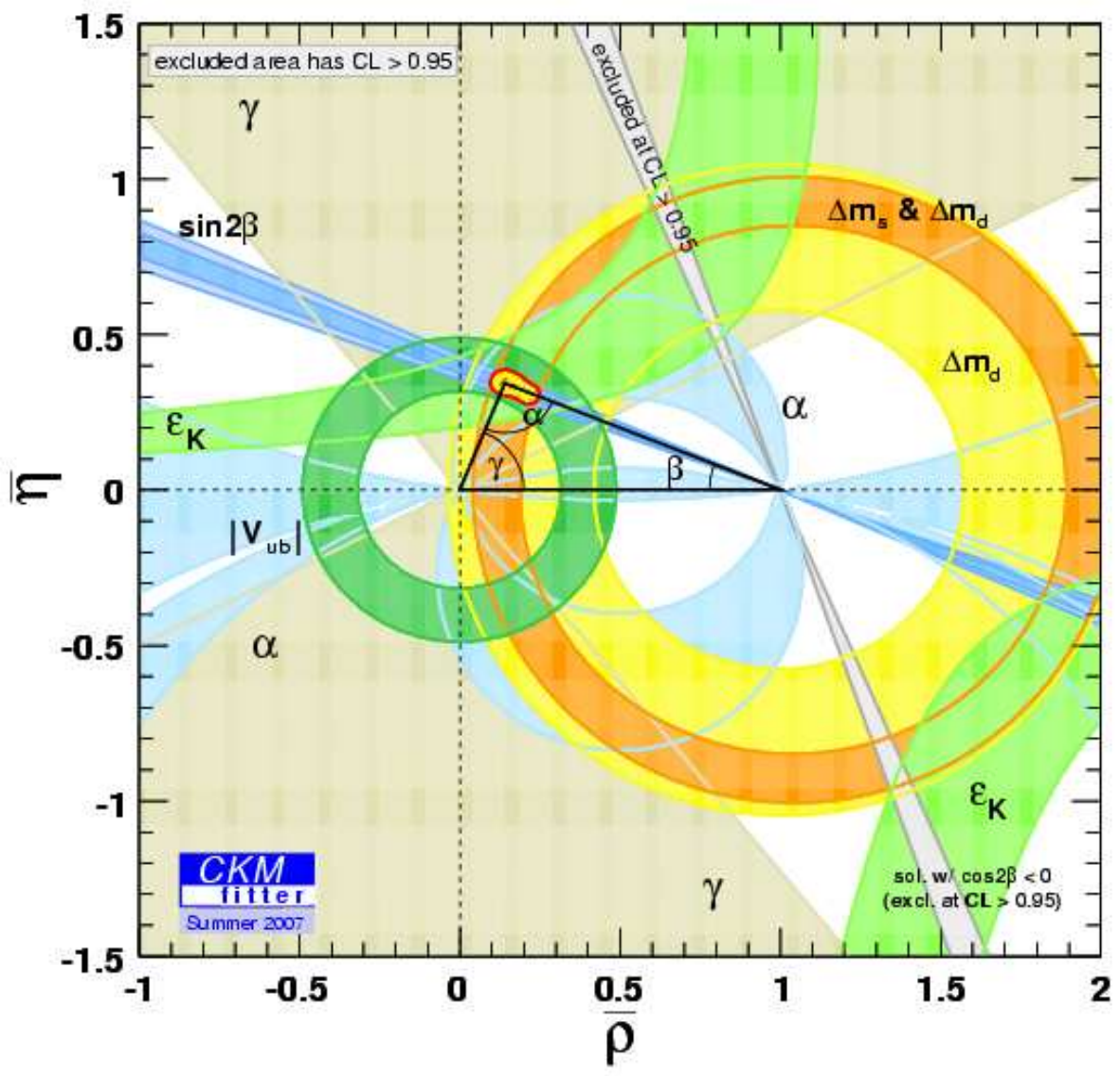

Figure 1.4. The current understanding of the unitarity triangle. This includes limits on the angles and sides of the triangle.

Currently, the CKM matrix element with the largest uncertainty, assuming unitarity, is $V_{t d}$, as evident from Equation 1.8 and Figure 1.4. This error can be reduced by either a measurement of $\Delta m_{s}$ or by placing lower limits on the $B_{s}^{0}$ oscillation frequency, as 
mentioned above; Section 1.1.4 will explain this indirect probe approach. Additionally, the uncertainty on $V_{t d}$ can be reduced by improvements in the theoretical calculations of the decay constants and the Bag parameters for $B_{s}^{0}$ and $B_{d}^{0}$; see Equation 1.28 and the explanation that follows.

\subsection{4. $B_{s}^{0}$ Mixing Formalism}

$B_{s}^{0}$ mixing occurs via the weak interaction, through six possible Feynman box diagrams. These box diagrams are shown in Figure 1.2. A $B_{s}^{0}$ mixes, or oscillates, into a $\overline{B_{s}^{0}}$ by exchange of two $W$ bosons and two quarks. Because the top quark is much, much heavier than the other quarks, it dominates this interaction. Therefore, $V_{t b}$ and $V_{t s}$ become the only contributing CKM matrix elements, and the six possible box diagrams are reduced to the two diagrams involving the top quark.

The $B_{s}^{0}$ and $\bar{B}_{s}^{0}$ correspond to the weak eigenstates in quantum mechanics. The strong, or mass, eigenstates are denoted as $B_{L}$ and $B_{H}$ for the light and heavy mass states, respectively. The $B_{L}$ eigenstate is about equal to the CP-odd state while the $B_{H}$ corresponds to the $\mathrm{CP}$-even state; in the absence of $\mathrm{CP}$ violation, the mass states are equal to the $\mathrm{CP}$ eigenstates.

The mass eigenstates are related to the weak eigenstates by the following equations:

$$
\begin{aligned}
& \left|B_{L}\right\rangle=p\left|B_{s}^{0}\right\rangle+q\left|\bar{B}_{s}^{0}\right\rangle, \\
& \left|B_{H}\right\rangle=p\left|B_{s}^{0}\right\rangle-q\left|\bar{B}_{s}^{0}\right\rangle,
\end{aligned}
$$


where

$$
\frac{q}{p}=\sqrt{\frac{M_{12}^{*}-\frac{i}{2} \Gamma_{12}^{*}}{M_{12}-\frac{i}{2} \Gamma_{12}}}=e^{-i \phi}
$$

and where $M_{12}$ and $\Gamma_{12}$ are elements of the mass matrix.

Similarly, the weak eigenstates are related to the mass eigenstates by the equations:

$$
\begin{aligned}
\left|B_{s}^{0}\right\rangle & =\frac{1}{2 p}\left(\left|B_{L}\right\rangle+\left|B_{H}\right\rangle\right), \\
\left|\bar{B}_{s}^{0}\right\rangle & =\frac{1}{2 q}\left(\left|B_{L}\right\rangle-\left|B_{H}\right\rangle\right),
\end{aligned}
$$

where $q / p$ is defined as above.

The masses of the heavy and light mass eigenstates are related to the mass of the $B_{s}^{0}$ and the mass difference of the heavy and light states, as follows:

$$
\begin{gathered}
m_{H}=M_{B_{s}}+\Delta m_{s}, \\
m_{L}=M_{B_{s}}-\Delta m_{s}
\end{gathered}
$$

where $M_{B_{s}}$ is the mass of the $B_{s}^{0}$ meson, and $\Delta m_{s}$ is half the mass difference between the heavy and light mass eigenstates, written as: $\Delta m_{s}=\frac{1}{2}\left(m_{H}-m_{L}\right)$. The Standard Model predicts the $B_{s}^{0}$ oscillation frequency to be within a certain range. This range varies depending on which theoretical prediction is used, but is generally accepted to be within 15 to $25 \mathrm{ps}^{-1}$. If $\Delta m_{s}$ were to lie outside this range, new physics would have to be used to explain the frequency. 
Working out the Feynman box diagrams, using the notation and derivation of Gay [11], the matrix element can be found to be proportional to:

$$
\mathcal{M} \propto V_{t s}^{*} V_{t b} V_{t b}^{*} V_{t s} \bar{s} \gamma^{\mu}\left(1-\gamma^{5}\right) W_{\mu \nu}^{ \pm} \gamma^{\nu}\left(1-\gamma_{5}\right) b
$$

where

$$
W_{\mu \nu}^{ \pm}=\frac{-i\left(g_{\mu \nu}-q_{\mu} q_{\nu} / m_{W}^{2}\right)}{q^{2}-m_{W}^{2}}
$$

This reduces to the mass difference, $\Delta m_{s}$, as:

$$
\Delta m_{s}=2\left|M_{12}\right|=\frac{\mathcal{M}}{m_{B_{s}}}=\frac{G_{F}^{2}}{3 \pi^{2}} m_{B_{s}} f_{B_{s}}^{2} B_{B_{s}} \eta_{B} m_{t}^{2} f_{2}\left(\frac{m_{t}^{2}}{m_{W}^{2}}\right)\left|V_{t s}^{*} V_{t b}\right|^{2}
$$

where:

- $G_{F}$ is the Fermi constant,

- $m_{B_{s}}$ is the mass of the $B_{s}^{0}$-meson,

- $f_{B_{s}}$ is the $B_{s}$ decay constant,

- $B_{B_{s}}$ is the Bag parameter,

- $\eta_{B}$ is the QCD parameter,

- $m_{t}$ is the mass of the top quark,

- $f_{2}$ is the Inami-Lim function, and

- $m_{W}$ is the mass of the $W$ boson.

The Fermi constant is proportional to the square of the electroweak coupling constant $g$, as: $G_{F}=\frac{\sqrt{2} g^{2}}{8 m_{W}^{2}}$. The $B_{s}^{0}$ decay constant, $f_{B_{s}}$, is determined from lattice QCD [12]; the current best calculation is $f_{B_{s}} / f_{B}=1.20 \pm 0.03$ (stat + chiralfit) \pm 0.01 (other) from the HPQCD Collaboration[13. The Bag parameter is essentially a "fudge" factor that 
comes from collapsing the sum over all states in the matrix element calculation to only include the vacuum state. $B_{B_{s}}$ then corrects for the exclusion of intermediate states; if the vacuum state was the only contributing state, then $B_{B_{s}}=1$. The current "best" calculation for $B_{B_{s}}$, as stated by Okamoto[12], is from the JLQCD Collaboration [14] using unquenched lattice QCD calculations; their result is $B_{B_{s}} / B_{B_{d}}=1.017 \pm 0.016_{-0.017}^{+0.056}$. The QCD correction factor, $\eta_{B}$, accounts for any number of gluons added to the mixing box diagrams. By definition, $\eta_{B}$ is the same for $B_{s}^{0}$ and $B_{d}^{0}$ mesons 11], and the standard calculation was done by Buras, Jamin and Weisz [15] using next-to-leading order (NLO) QCD. The Inami-Lim function [16] describes the calculation of the internal loop, or box, of the diagram.

The equation above for $\Delta m_{s}$ on its own has a theoretical uncertainty of about $15 \%[\mathbf{1 2}$. This comes mainly from the uncertainty on the decay constant. However, if you take the ratio of $\Delta m_{s}$ to $\Delta m_{d}$, many of the theoretical parameters cancel and the theoretical uncertainty is reduced to the order of $3 \%$, as shown here:

$$
\frac{\Delta m_{s}}{\Delta m_{d}}=\frac{m_{B_{s}} f_{B_{s}}^{2} B_{B_{s}}}{m_{B_{d}} f_{B_{d}}^{2} B_{B_{d}}}\left|\frac{V_{t s}}{V_{t d}}\right|^{2} .
$$

The ratio of $B$ decay constants leads to the cancellation of the uncertainty from perturbation theory $\mathbf{1 3}$, and the ratio of Bag parameters cancels out a number of systematic uncertainties in the lattice QCD calculations [14. The current measurements of $V_{t s}[\mathbf{6}$ come from measurements of the inclusive rate $B \rightarrow X_{s} \gamma[\mathbf{9}$ and have an uncertainty of about $2 \%$, as seen in Equation 1.8 .

The CKM matrix element $V_{t d}$ can be more precisely determined from this ratio, because of the reduction in theoretical uncertainty. $\Delta m_{d}$ has been precisely determined $[\mathbf{6}$ 
and so a measurement of $\Delta m_{s}$ can be used in the ratio to provide a precise determination of $V_{t d}$. Until recently, the error on the CKM matrix element $V_{t d}$ was the largest uncertainty in this frequency ratio and was limited by experimental uncertainty. After D-Zero's two-sided bound [17] and the CDF's measurement [10], this ratio is now limited by the theoretical uncertainty. After CDF's measurement, the limits on the CKM elements from HFAG 9 are:

$$
\frac{\left|V_{t d}\right|}{\left|V_{t s}\right|}=\xi \sqrt{\frac{\Delta m_{d}}{\Delta m_{s}} \frac{m\left(B_{s}^{0}\right)}{m\left(B_{d}^{0}\right)}}=0.2062 \pm 0.0011_{-0.0060}^{+0.0080},
$$

where $\xi=1.210_{-0.035}^{+0.047}[\mathbf{1 2}$.

\subsection{Overview of This Analysis}

The analysis described in this thesis was done using a single $B_{s}^{0}$ decay channe 1 , $B_{s}^{0} \rightarrow D_{s}^{-} \mu^{+} \nu X, D_{s}^{-} \rightarrow \phi \pi^{-}$; a diagram of this decay is shown in Figure 1.5. This is called a semileptonic channel, since the $B_{s}^{0}$ decays into a lepton (the muon) and the $D_{s}^{-}$decays hadronically. The neutrino in the final state cannot be reconstructed, and the momentum carried away by the neutrino must be accounted for. This is done using a scaling factor called a $K$ factor, described in detail in Section 3.6.2. Other decay channels under study at D-Zero are the semileptonic decays $B_{s}^{0} \rightarrow D_{s}^{-} e^{+} \nu X\left(D_{s}^{-} \rightarrow \phi \pi^{-}\right)$, $B_{s}^{0} \rightarrow D_{s}^{-} \mu^{+} \nu X \quad\left(D_{s}^{-} \rightarrow K^{0 *} K^{-}\right)$, and $B_{s}^{0} \rightarrow D_{s}^{-} \mu^{+} \nu X \quad\left(D_{s}^{-} \rightarrow K_{S}^{0} K^{-}\right)$, as well as the hadronic channel $B_{s}^{0} \rightarrow D_{s}^{-} \pi^{+} X\left(D_{s}^{-} \rightarrow \phi \pi^{-}\right)$. Details of these analyses are not presented here, but are documented in References [18], 19], 20], and [21], respectively, and their combination is discussed in Reference [22].

\footnotetext{
${ }^{1}$ Charge conjugate states are implied throughout this thesis.
} 


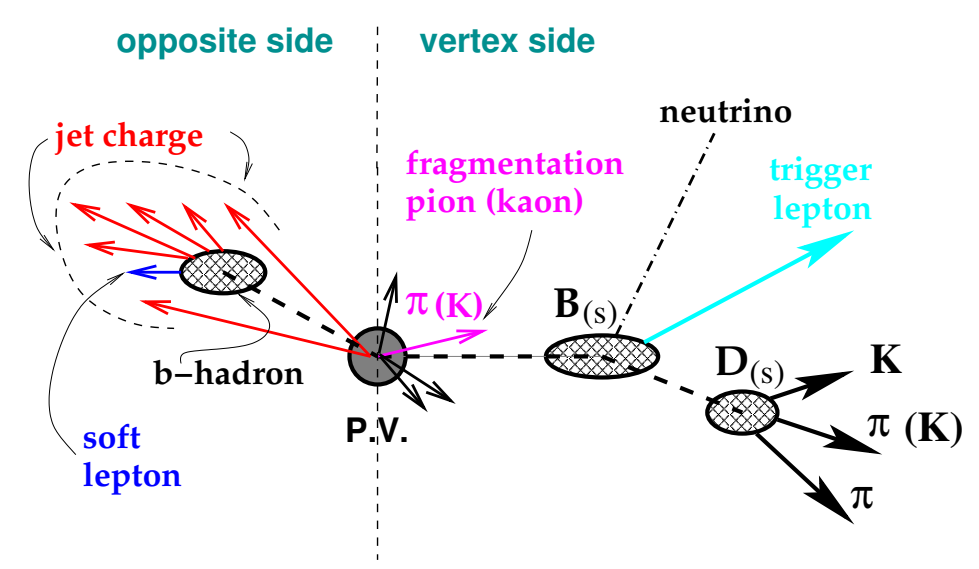

Figure 1.5. An example of a $B_{s}^{0} \rightarrow \mu \phi \pi$ decay. At the dashed line is the collision point, labeled "P.V.". To the right is the $B_{s}^{0}$ event, which decays into a $D_{s}^{-}$, a muon (labeled "trigger lepton"), and a neutrino which is not reconstructed. The $D_{s}^{-}$decays into a $\phi$ (the two $K \mathrm{~s}$ ) and a pion. To the left of the dashed vertical line is another $b$-hadron; the importance of this side of the event is discussed later in this thesis. Diagram courtesy of Sasha Rakitin.

The $B_{s}^{0} \rightarrow D_{s}^{-} \mu^{+} \nu X, D_{s}^{-} \rightarrow \phi \pi^{-}$channel has been studied previously at D-Zero, leading to the first upper-bound on $\Delta m_{s}$ [17. This thesis expands and improves on that analysis, with the addition of a number of software improvements to the fit inputs, discussed in Chapter 31, as well as using over two and a half times the data of the original analysis. Additionally, the LayerØ hardware upgrade to the D-Zero detector in early 2006, discussed in Section 2.2.2.1 improved the quality of the data collected after its installation.

All $B$ mixing analyses have the same basic steps. A $B_{s}^{0}$ mixing analysis can be summarized as an asymmetry measurement, since the asymmetry of the number of oscillated and non-oscillated $B_{s}^{0}$ events is related to the oscillation frequency of the $B_{s}^{0}$ meson. To determine if the $B_{s}^{0}$ oscillated, we check if the meson at production was a $B_{s}^{0}$ or $\bar{B}_{s}^{0}$ and whether it was a $B_{s}^{0}$ or $\bar{B}_{s}^{0}$ at decay. If it was a $B_{s}^{0}\left(\bar{B}_{s}^{0}\right)$ at both production and decay, 
then it did not oscillate. Conversely, if it was a $B_{s}^{0}\left(\bar{B}_{s}^{0}\right)$ at production and a $\bar{B}_{s}^{0}\left(B_{s}^{0}\right)$ at decay, then it oscillated. A $B_{s}^{0}$ that oscillated is referred to as "mixed" and one that did not oscillate is called "unmixed".

For $B_{s}^{0}$ mixing, the asymmetry, as a function of time, is defined as:

$$
\mathcal{A}\left(t_{B_{s}^{0}}\right)=\frac{N_{u}(t)-N_{m}(t)}{N_{u}(t)+N_{m}(t)},
$$

where $u$ and $m$ abbreviates "unmixed" and "mixed", respectively. If a measurement of $\Delta m_{s}$ is possible, then the asymmetry distribution can be fit with a cosine, and the amplitude of the cosine will be equal to the amplitude obtained using the Amplitude Method.

The first step in the analysis is to reconstruct the particles in a given decay channel and then find the primary and secondary vertices of the decay. The momentum of the $B$-meson gives the proper time, according to the relation:

$$
t=\frac{L_{x y} m_{B}}{p_{T}},
$$

where $L_{x y}$ is the transverse decay length, $m_{B}$ is the mass of the $B$-meson, and $p_{T}$ is the transverse momentum of the particle measured in the $x-y$ plane of the detector.

Next, we determine the flavor of the $B_{s}\left(B_{s}^{0}\right.$ or $\left.\overline{B_{s}^{0}}\right)$ at production and decay, establishing whether the $B_{s}$-meson oscillated or not. We also measure the decay length of the $B_{s}^{0}$, as the probability of oscillation is a function of decay length. It should be noted that the $B_{s}^{0}$ meson has a finite decay length with an average decay length of $439 \mu \mathrm{m}$ [6], and we measure the decay length as the distance between the primary vertex, where the $B_{s}^{0}$ is 
produced, and the secondary vertex, where the $B_{s}^{0}$ decays; the production and decay vertices are shown in Figure 1.5. Other inputs are included and are described in more detail in Section 3.6. We fit for $\Delta m_{d}$, the $B_{d}^{0}$ oscillation frequency introduced in Section 1.1.3, in order to verify our procedure. Finally, we fit for $\Delta m_{s}$ to obtain a result. The details of each step in the analysis are described in Chapter 3 , 


\section{CHAPTER 2}

\section{Experimental Apparatus - Tevatron and the D-Zero Detector}

The data used for this analysis was collected at the D-Zero detector, one of two collider detectors at the Tevatron at Fermi National Accelerator Laboratory (Fermilab) in Batavia, Illinois.

\subsection{The Tevatron at Fermilab}

The Tevatron is a ring four miles in circumference and just over one mile across (1 $\mathrm{km}$ radius). Inside the ring, a beam of protons and a beam of anti-protons are circulated

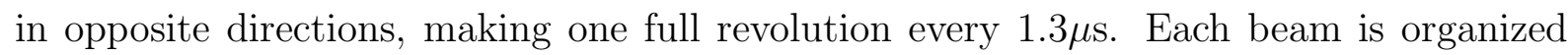
into 36 bunches and collisions occur every 396ns. The center-of-mass energy of the beam is $\sqrt{s}=1.96 \mathrm{TeV}$. The two beams collide together at two points along the ring where detectors reside. The two Tevatron detectors are the Collider Detector at Fermilab (CDF) and the D-Zero detector.

\subsection{The D-Zero Detector}

The D-Zero detector is a multipurpose detector consisting of three main systems: a tracking system, a calorimeter, and muon detectors. A brief overview is provided here. The most important detector systems for $B$-physics analyses are the tracking and muon systems. Those are described in more detail in the sections that follow. For a more complete description, see Reference [23]. 
The D-Zero detector was designed to study high- $p_{T}$, or high transverse momentum, physics and large-mass particles. Data collected prior to 1997 is called RunI and is not discussed here. Data collected after March 2001 is called RunII, subdivided into RunIIa which ran from March 2001 to March 2006, and RunIIb which started in June 2006 and is still ongoing at the time of this thesis submission.

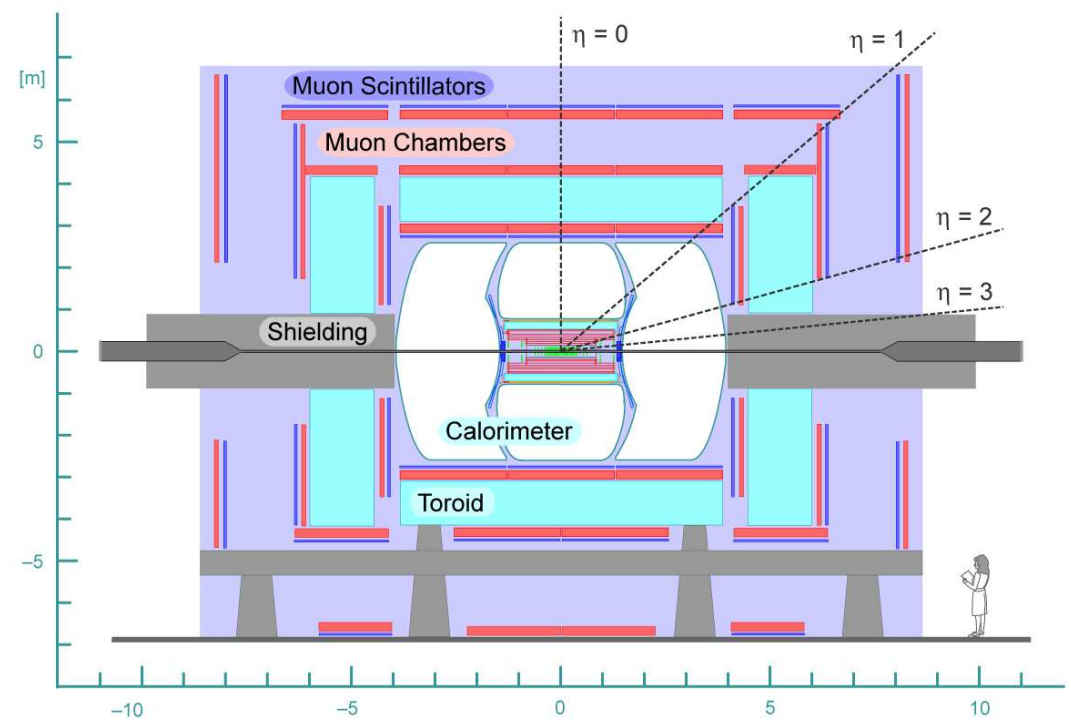

Figure 2.1. The RunII D-Zero detector. The tracking system is the innermost detector, followed by the solenoid and calorimeter. Next, the inner muon detectors, the toroid, and finally the outer muon chambers. A closeup of the tracking systems is shown in Figure 2.2.

The detector was significantly upgraded before the start of RunIIa. The central tracking system was completely replaced with a more sophisticated system, and is now located inside a $2 \mathrm{~T}$ solenoid magnet to obtain particle charge information. It is described in more detail in Section 2.2.2 Preshower detectors were added as well, between the tracking system and the calorimeter, and the muon system was upgraded to better prevent radiation damage. In addition, the D-Zero detector was upgraded during the 2006 shutdown just 
prior to the start of RunIIb. During that shutdown, an additional silicon layer was added to the silicon tracking system. This was the main hardware upgrade of the 2006 shutdown and is discussed in more detail at the end of Section 2.2.2. The D-Zero trigger system was also upgraded to handle the expected increases in luminosity while keeping within data bandwidth constraints.

\subsubsection{Coordinate System at D-Zero}

The coordinate system used at the D-Zero detector should be noted. Cartesian coordinates are defined such that the $z$-axis is aligned with the proton beampipe, the $x$-axis points toward the center of the Tevatron ring, and the $y$-axis is vertical. The D-Zero detector is centered at $(0,0,0)$. The polar and azimuthal angles are defined respectively as:

$$
\theta=\tan ^{-1}\left(\frac{r}{z}\right) \text { and } \phi=\tan ^{-1}\left(\frac{y}{x}\right)
$$

where $r$ is measured as the perpendicular distance from the beampipe.

Finally, pseudorapidity, is defined as:

$$
\eta=-\ln \left(\tan \left(\frac{\theta}{2}\right)\right)
$$

where $\Delta \eta$ is Lorentz-invariant.

\subsubsection{Silicon Microstrip Tracker System}

For $B$-physics analyses, it is important to precisely determine the particle's point of production and decay. These are known as the primary (PV) and secondary (SV) vertices, respectively. The D-Zero detector's vertexing capability is greatly enhanced by a silicon 
tracking system. Silicon-based tracking provides good hit resolution and was placed close to the interaction region for better resolution.

The D-Zero tracking system consists of the Silicon Microstrip Tracker (SMT) system, Silicon Track Trigger (STT), Central Fiber Tracker (CFT), Central Preshower (CPS) and Forward Preshower (FPS), and the Central Track Trigger (CTT). The SMT, CFT, CPS and FPS are all hardware systems. The STT uses information from the SMT and CTT to make Level2 trigger decisions, while the CTT uses information from the CFT, CPS and FPS to make both Level1 and Level2 trigger decisions. The trigger system at D-Zero is discussed in Section 2.2.7. More information on the STT can be found in Reference 24] and more information on the CTT can by found in Reference [25].

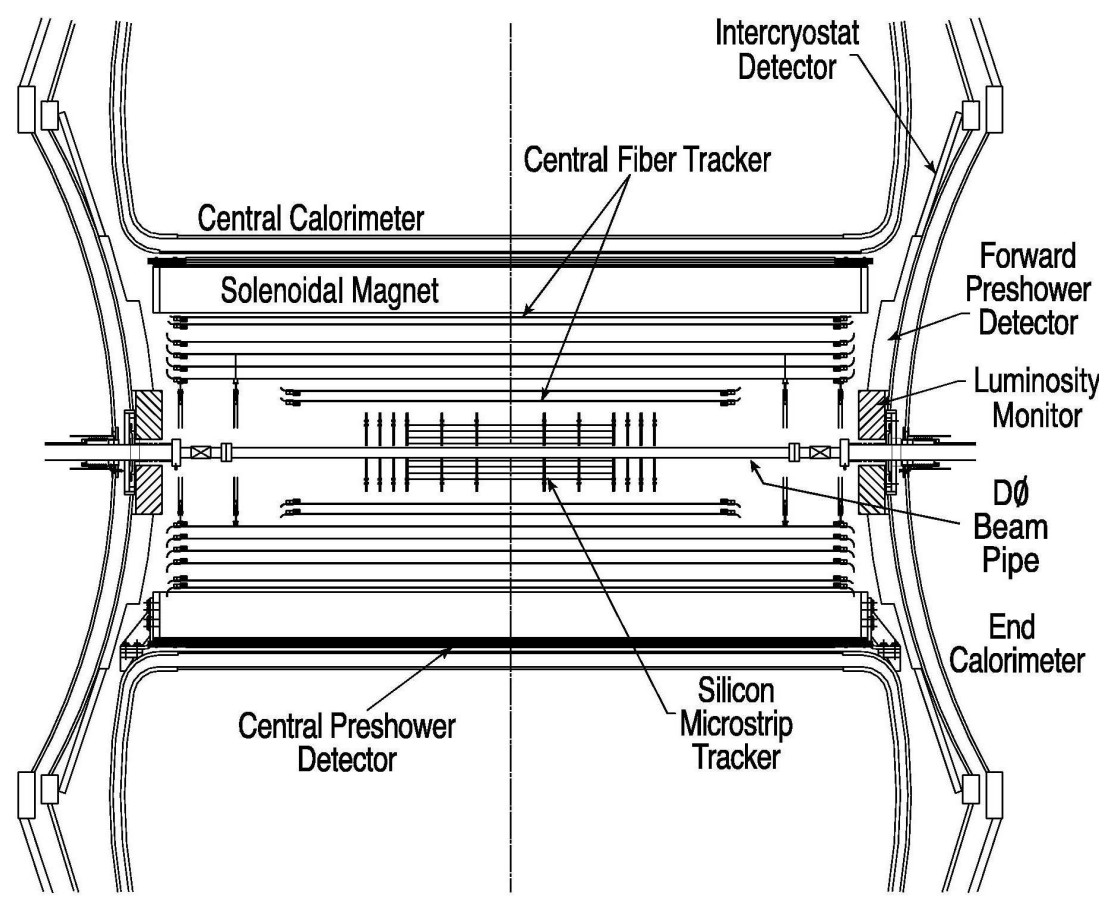

Figure 2.2. The RunII D-Zero tracking systems, showing the Silicon Microstrip Tracker, Central Fiber Tracker, solenoid, and the preshower detectors. 23 ] 
The Silicon Microstrip Tracker (SMT) is the innermost section of the D-Zero detector. Particles pass through the silicon wafers, and the SVX2 readout chips of 128 channels each carry out the particle's information. It is a hybrid system as it is composed of both barrels and disk detectors, called F-disks and H-disks. The RunIIa SMT detector was comprised of six $12 \mathrm{~cm}$ long barrels in four layers with F-disk detectors in between the barrel sections and four large-area H-disk detectors, two at each of the far ends of the barrels. The H-disks provide coverage up to pseudo-rapidity of $|\eta|<3$. Figure 2.3 shows the layout of the RunIIa SMT silicon system. A more detailed description of the RunIIa SMT system can be found in Reference [26].

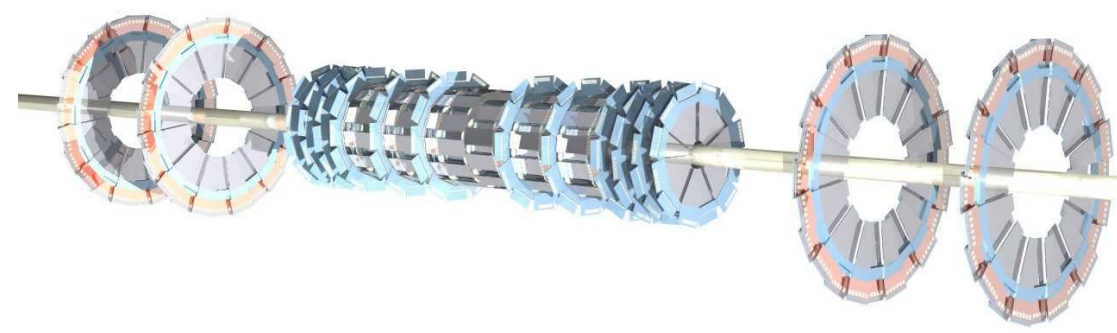

Figure 2.3. The layout of the RunIIa SMT system. There are three components: barrels, F-disks, and H-disks. The barrels are parallel to the beampipe, and the F-disks are spaced in between the barrels. The H-disks are the four larger-area disks at the outer ends of the silicon system. 223

2.2.2.1. The Layer $\varnothing$ Detector. For the RunIIb SMT system, a new barrel layer of silicon, called Layer $\varnothing$, was added, fitting around the beampipe and inside the RunIIa SMT silicon layers at a distance of $1.67 \mathrm{~cm}$ from the interaction region. In addition, the outermost H-disks on either end of the SMT were removed. This was done to provide readout electronics for LayerØ by sacrificing the least-important SMT disks, as installation of new electronics was not possible due to physical logistics in the D-Zero collision hall. 
A picture of Layer $\varnothing$ is shown in Figure 2.4 and details of the RunIIb SMT Upgrade, LayerØ detector, and a detailed overview of the RunIIa SMT system can be found in Reference [27].

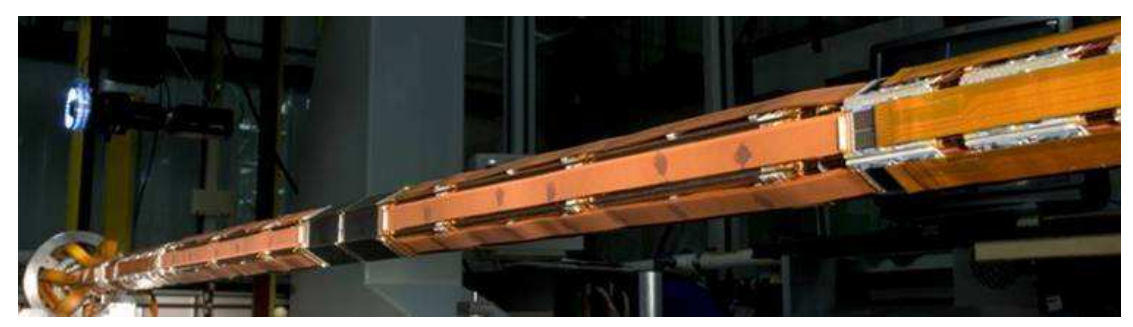

Figure 2.4. The Layer $\varnothing$ detector, showing the silicon sensors at the center, with the readout electronics carrying signals outside the detector region.

LayerØ uses SVX4 readout chips, whereas the RunIIa SMT system is comprised of SVX2 readout chips. The SVX4 chips are more stable and more radiation hard than the SVX2 chips. In addition, Layer $\varnothing$ provides approximately a $30 \%$ improvement in single-hit resolution, making LayerØ an important improvement over the RunIIa SMT detector.

Layer $\varnothing$ is comprised of 48 single-sided silicon sensors of 256 channels each. Note that the $\mathrm{F}$ and H-disks of the original SMT system are made from double-sided silicon sensors. Layer $\varnothing$ has an inner layer (the "A" layer) at $r=16.0 \mathrm{~mm}$ and an outer layer (the "B" layer) at $r=17.6 \mathrm{~mm}$; the edges of the two layers slightly overlap to maximize the acceptance of LayerØ. Each of the sensors is attached to a hybrid of two $0.25 \mu \mathrm{m}$ silicon SVX4 chips using a low-mass analog cable. Signals from the sensors are carried through the low-mass cables to the hybrid chips. From there, the signal is digitized and then sent using digital jumper cables connected to junction cards to adapter cards through twisted pair cables. The adapter cards allow the voltage requirements of the SVX4 chips to interface to the remaining electronics, as the voltage requirements of the SVX2 chips 
are different. The electronics that were used by the outer H-disks removed during the 2006 shutdown make up the remaining readout electronics for LayerØ. More detailed information about Layer $\varnothing$ can be found in Reference [28].

\subsubsection{Central Fiber Tracker}

The Central Fiber Tracker (CFT) system is composed of layers of wavelength-shifting (WLS) fibers. There are eight concentric layers attached to carbon fiber support cylinders. Note that the inner layers are shorter in $z$ than the outer layers; this is to accommodate the H-disks in the SMT and the effect can be seen in Figure 2.2 Each of the eight layers is made up of an axial layer of WLS fibers with a stereo layer of fibers on top. An example of one of the eight layers is shown in Figure 2.5. The axial layers are parallel to the beam direction $z$, and the stereo layers are set $\pm 3^{\circ}$ off the $z$-axis; the stereo direction alternates with each of the eight layers. There are a total of 76,800 fibers in the CFT detector; each fiber is connected to an optical fiber and the optical fibers connect to Visible Light Photon Counters (VLPCs). VLPCs are similar to photo-multiplier tubes (PMTs) in that they convert light into pulses, but are solid-state and are kept in a cryogenic system.

The SMT and CFT can locate the primary vertex with a resolution of about $35 \mu \mathrm{m}$ in the $z$ direction, and can tag a $b$-quark jet having $p_{T}>10 \mathrm{GeV}$ and $|\eta|=0$ with an impact parameter resolution of less than $15 \mu \mathrm{m}$ in $r-\phi[\mathbf{2 3}]$. More information on the CFT system can be found in Reference [29]. 


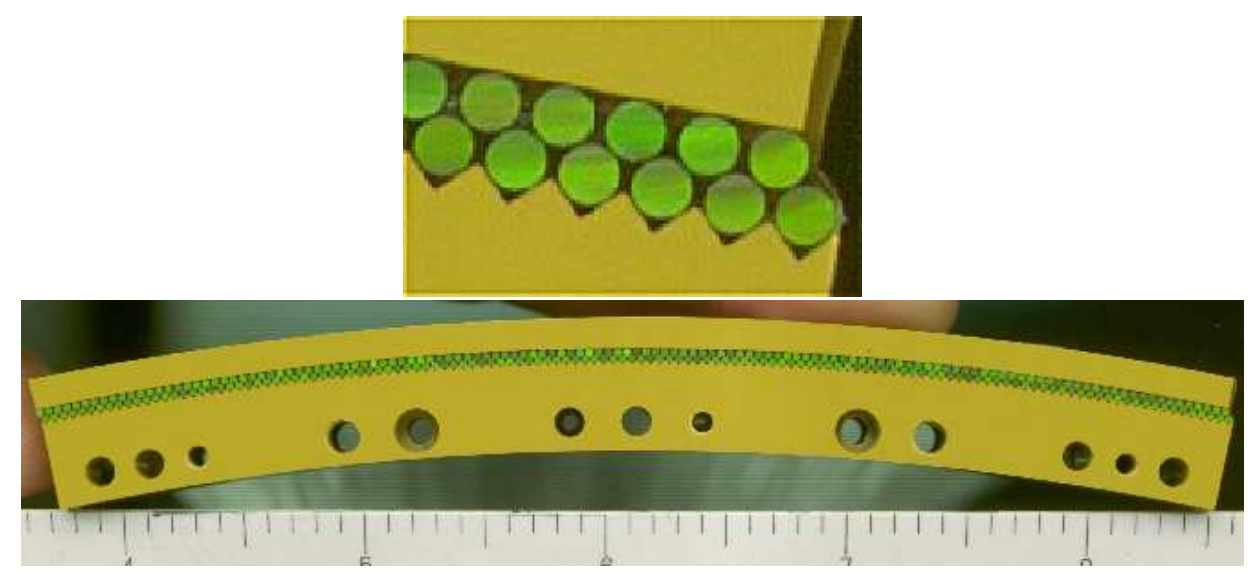

Figure 2.5. An example of a CFT layer, showing an end view of the WLS fibers. [30]

\subsubsection{Preshower Detectors}

The preshower detectors are placed just after the solenoid and just before the calorimeter. They are positioned such that the showers of electrons and photons beginning just before this detector, in the solenoid coil, are located and measured. The preshower detectors have much finer resolution than the calorimeter and thus assist in the tracking by indicating precisely where particles passed through the preshower detectors. The preshower detectors have three parts: there is a central preshower detector (CPS) with a range of $|\eta|<1.3$ and two forward preshower detectors (FPS) with a coverage of $1.5<|\eta|<2.5$. The preshower detectors are made of triangular strips of scintillator with WLS fibers running through their center to collect the light and sent it to PMTs. A detailed illustration is provided in Figure 2.6. The CPS is made up of an axial layer and two stereo layers, one at $+23^{\circ}$ and one at $-23^{\circ}$. The FPS is made up of two planes of scintillator strips. Detailed information about the preshower detectors can be found in Reference [31]. 


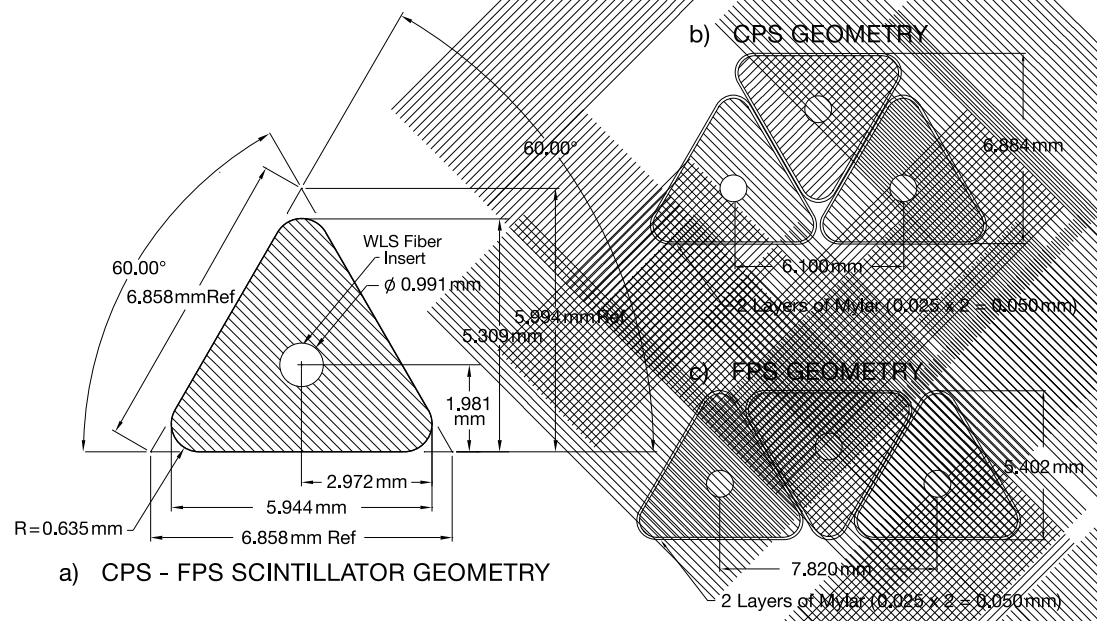

Figure 2.6. The D-Zero preshower detectors. [23]

\subsubsection{Calorimeter}

Outside of the solenoid and beyond the FPS, the D-Zero detector has a liquid-argon and uranium calorimeter, divided into three main sections: the central calorimeter (CC) and two endcap calorimeters (ECs). The CC covers the central region up to $|\eta|=1$, and the EC coverage is $1<|\eta|<4$. The calorimeters have a depth of about 20 radiation lengths, with around 4 radiation lengths of material (at $|\eta|=0$ ) from detector components between the interaction region and the first calorimeter layer [23. The innermost portion of each section is an electromagnetic calorimeter, followed by a hadronic calorimeter. The calorimeter is divided into smaller sections of $\phi$ and $z$ for better resolution. An illustration of the calorimeter is provided in Figure 2.7 and more detailed information about the calorimeter is given in Reference [32]. 


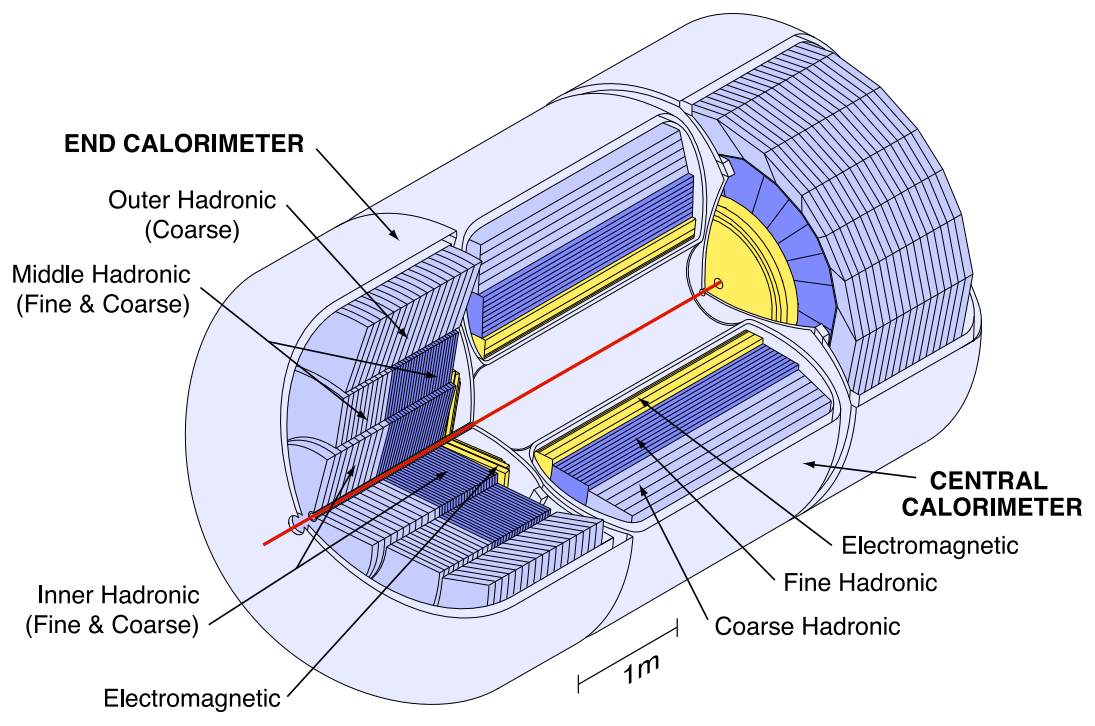

Figure 2.7. The D-Zero calorimeter. The electromagnetic portion is closest to the beam, with the fine and then coarse hadronic calorimeter following. 23 .

\subsubsection{Muon System}

The muon system is the outermost system of the D-Zero detector. It consists of a single layer of muon detectors (the "A" layer), followed by a $2 \mathrm{~T}$ toroid, made up of a central toroid and two end toroids. Beyond the toroids are an additional two layers (the "B" and "C" layers) of muon chambers. The muon system has an acceptance up to about $|\eta|=2$, and is made up of proportional drift tubes (PDTs) in the central region $(|\eta|<1)$, and mini-drift tubes (MDTs) for the forward region, where $1<|\eta|<2$. The MDTs are important particularly in the range $|\eta|>1.6$ where the central tracker coverage is not as good.

The muon system also uses scintillation counter systems, both inside and outside the toroids. This allows for better triggering and identification of muons, as well as rejection of cosmic rays, due to the precise timing information from the counters. Additionally, 


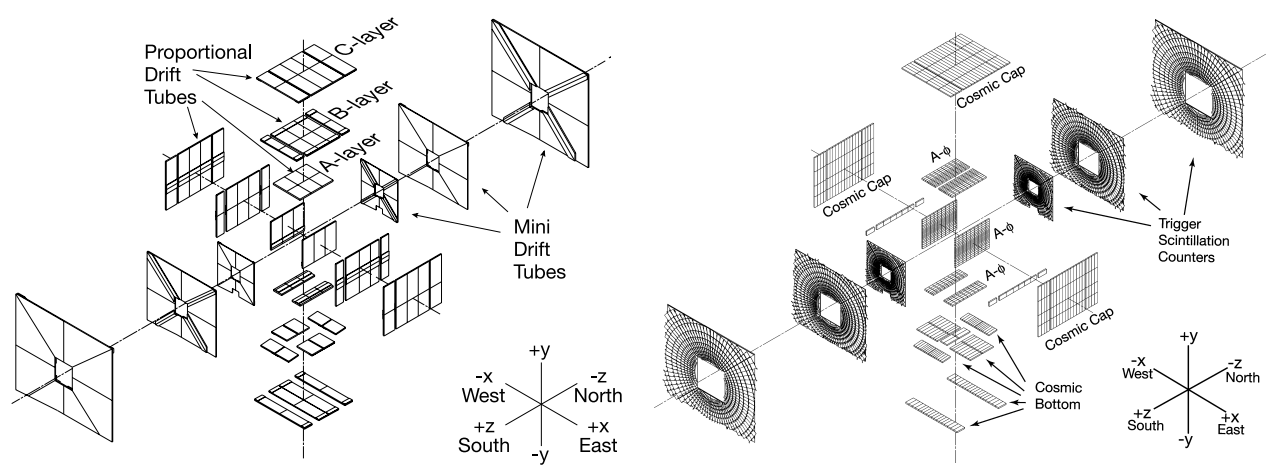

Figure 2.8. An exploded view of the D-Zero muon systems. The layout of the drift tubes is shown on the left, and the scintillation counters on the right. 23]

the muon system provides an independent measurement of the muon momentum from that provided by the tracking system. In the central region there are two scintillation counters, one before the toroid and one after. The muon momentum resolution in the central region is dominated by the SMT and CFT [33. In the forward region there are three sets of scintillation counters: one before the end toroid and two after the end toroid. The layout pattern of the scintillation counters matches that of the central fiber tracker in $\phi$, to match muon triggering requirements at Level1.

The muon system also has "pixel" counters in the forward region, on either side of the end toroids. The "A" and "B" layers lie directly inside and outside the toroid respectively, while the "C" layer is a few feet outside of the toroid. The scintillator wedges are arranged in an $r-\phi$ geometry, making the scintillator modules shaped like trapezoids. The $\phi$ segmentation matches that of the CFT, as described previously. In the forward region, about $50 \%$ of the muons triggered are reconstructed offline, and recorded muons have a purity of $80 \%$ and an efficiency of $90 \%$ in the forward region 33 . 


\subsubsection{Overview of the Trigger System at D-Zero}

The main points of the trigger system at D-Zero should also be noted. Tens of millions of collisions occur every second at D-Zero. A single event contains a large amount of data, and writing out all of the information from every event is not feasible. Instead, choices are made about which events are the more interesting ones, and then that event and its information is read out and saved. This set of choices is called the triggering system. It has a three-level decision process: Level1 (L1), Level2 (L2), and Level3 (L3). All information that passes L3 is written to tape for use in analyses.

The L1 trigger system is hardware-based, and makes decisions based on information from the CTT, calorimeter, and the muon system. At this level, the decision is based on a basic track algorithm using data from the CFT and preshower detectors, the energy deposited in the calorimeter, muon objects created from wire and scintillator hits as well as muon objects created from CTT tracks matched to hits in the muon scintillators. L1 reduces the rate of about $27 \mathrm{MHz}$ coming in from the detector to $2 \mathrm{kHz}$, and events passing L1 are digitized and sent to L2.

L2 makes more sophisticated decisions, and utilizes both hardware and software components. L2 uses data from the STT, CTT, CPS and FPS, calorimeter and muon systems. Decisions are made based on one of these systems or a combination of multiple systems. The STT uses SMT hit information and L1 CTT information, and the CTT uses both the STT and the L1 CTT information, calculating basic object kinematics. The preshowers also calculate basic kinematics, as well as flagging clusters with and without matched tracks as electrons and photons, respectively. The muon system improves muon identification with the use of calibration and more precise timing, and the calorimeter identifies 
jets (narrow cones of particles created from the hadronization of a quark), electrons, and photons.

Level3 of the trigger system takes the $1 \mathrm{kHz}$ rate coming from $\mathrm{L} 2$ and reduces it to an average recording rate of $50 \mathrm{~Hz}$, using more complex particle reconstruction algorithms. This level utilizes calibrations and definitions of physics objects to make its decisions. L3 selections are based on physics objects, such as muons and electrons, and their relation to other physics objects, such as the distance between two objects or the combined mass. The events passing L3 requirements are written to tape.

The triggering system uses a list of definitions called a trigger list. An individual trigger might be defined to find high-quality muons with a $p_{T}>3 \mathrm{GeV} / c$, for example. Many analyses require that their events were triggered by a certain trigger or set of triggers to help increase the quality of their data sample. For this analysis, no explicit trigger requirements are made; however, almost all events are recorded by single-muon triggers. The potential bias from triggers with an impact parameter cut is discussed in Section 4.2 .2

Another aspect of the triggering system is related to the luminosity of the beam. At high luminosities, it would be impossible for example, to write out every event that had a muon in it. To address this, many triggers are prescaled at high luminosities. A trigger prescale determines what fraction of events that pass that trigger will actually be written out. A prescale of 5 means that only one in 5 of the events passing those trigger requirements is saved. However, since saving the 5th, 10th, 15th, etc. event could produce a bias in the luminosity measurement, the choice of which event to save is made randomly such that overall, $20 \%$ of the events are written out. The D-Zero Trigger Board [34 
and Trigger Meisters [35] continually improve the triggers and attempt to reduce trigger prescales as much as possible. More information about the D-Zero trigger system can be found in Reference 36 .

One additional note should be made. The main difference between the triggering at D-Zero and the trigger system at CDF is the bandwidth. CDF has enough bandwidth at Level1 to have triggers that are track-based, and are therefore better able to trigger on fully-reconstructible hadronic $B_{s}^{0}$ decays. 


\section{CHAPTER 3}

\section{Analysis}

\subsection{Historical Perspective}

Historically, studies of mixing in neutral systems have yielded important and often unexpected results. Mixing was first discovered in the neutral Kaon system and first predicted by Gell-Mann and Pais in 1955 [37] . Originally, physicists believed the heavy and light Kaon states, known as $K_{S}$ and $K_{L}$ respectively, to be two completely different particles. It was Cabibbo [3] who proposed the weak mixing angle $\theta_{C}$ and the rotation matrix in Equation 1.2] in 1963 to account for the decay rates of strange particles. Information on $\mathrm{CP}$ violation was gained when the mixing in the Kaon system was discovered [38], as the two Kaon states correspond to two different CP states. The first evidence for mixing in the $B$ system was found by UA1 in 1987 [39]. Measuring the oscillation frequency $\Delta m_{d}$ of the $B_{d}^{0}$ system [40, 41] told physicists that the top quark was much heavier than what had been predicted by theory. Within the last decade, the discovery of neutrino mixing proved conclusively that neutrinos have mass [42. Most recently, $D^{0}$ mixing results have come from the B-factories [43. Measuring the $B_{s}^{0}$ oscillation frequency is equally important as these previously studied systems, providing a precision test of the SM by indirectly constraining the CKM triangle when combined with the $\Delta m_{d}$ results. 
Table 3.1. Mixing in the Standard Model

\begin{tabular}{|c|c|c|}
\hline Particle & Mixing Allowed & Status \\
\hline$K^{0}$ & Yes & Observed \\
\hline$D^{0}$ & Yes & Observed \\
\hline$B_{d}^{0}$ & Yes & Observed \\
\hline$B_{s}^{0}$ & Yes & Observed \\
\hline$\pi^{0}$ & No & Is its own anti-particle \\
\hline$t$-quark & No & Too heavy to form stable hadrons before decaying \\
\hline Excited Mesons & No & Decays strongly before mixing can occur \\
\hline Neutrinos & Yes & Observed \\
\hline
\end{tabular}

\subsubsection{Previous Limits and Current Understanding of $\Delta m_{s}$}

While $B_{s}^{0}$ mixing had been proposed theoretically, it was not until the early 1990's when the first limits were put on the $B_{s}^{0}$ oscillation frequency by the LEP experiment $\Delta m_{s}$ 44, [45. Since then, only lower-limits have been placed on $\Delta m_{s}$, until the recent results from Fermilab's two collider experiments. $B_{s}^{0}$ mixing is difficult to study at the collider experiments, in part because the meson is produced much less frequently than the $B_{d}^{0}$ meson. Additionally, D-Zero did not have a solenoid until the start of RunII in 2002, effectively making mixing studies impossible. However, as D-Zero is currently the only experiment in the world studying $B_{s}^{0}$ mixing, the challenges are worth overcoming.

In the spring of 2006, D-Zero placed upper limits on the $B_{s}^{0}$ mixing frequency [17], followed weeks later by the CDF collaboration result [46]. By placing an upper-bound on $\Delta m_{s}$ of $21 \mathrm{ps}^{-1}$, D-Zero effectively ruled out all new physics models with large SM deviations at the $95 \%$ C.L. and greatly reduced the uncertainty on the relevant side of the Unitarity Triangle. By the end of 2006, CDF had measured $B_{s}^{0}$ mixing at a value of $17.77 \pm 0.10$ (stat) \pm 0.07 (sys) $\mathrm{ps}^{-1}$ [10. Figures 3.1, 3.2. and 3.3 show the unitarity 
triangle limits before the D-Zero two-sided bound, after the D-Zero result was included, and after both the D-Zero and CDF results were included, respectively.

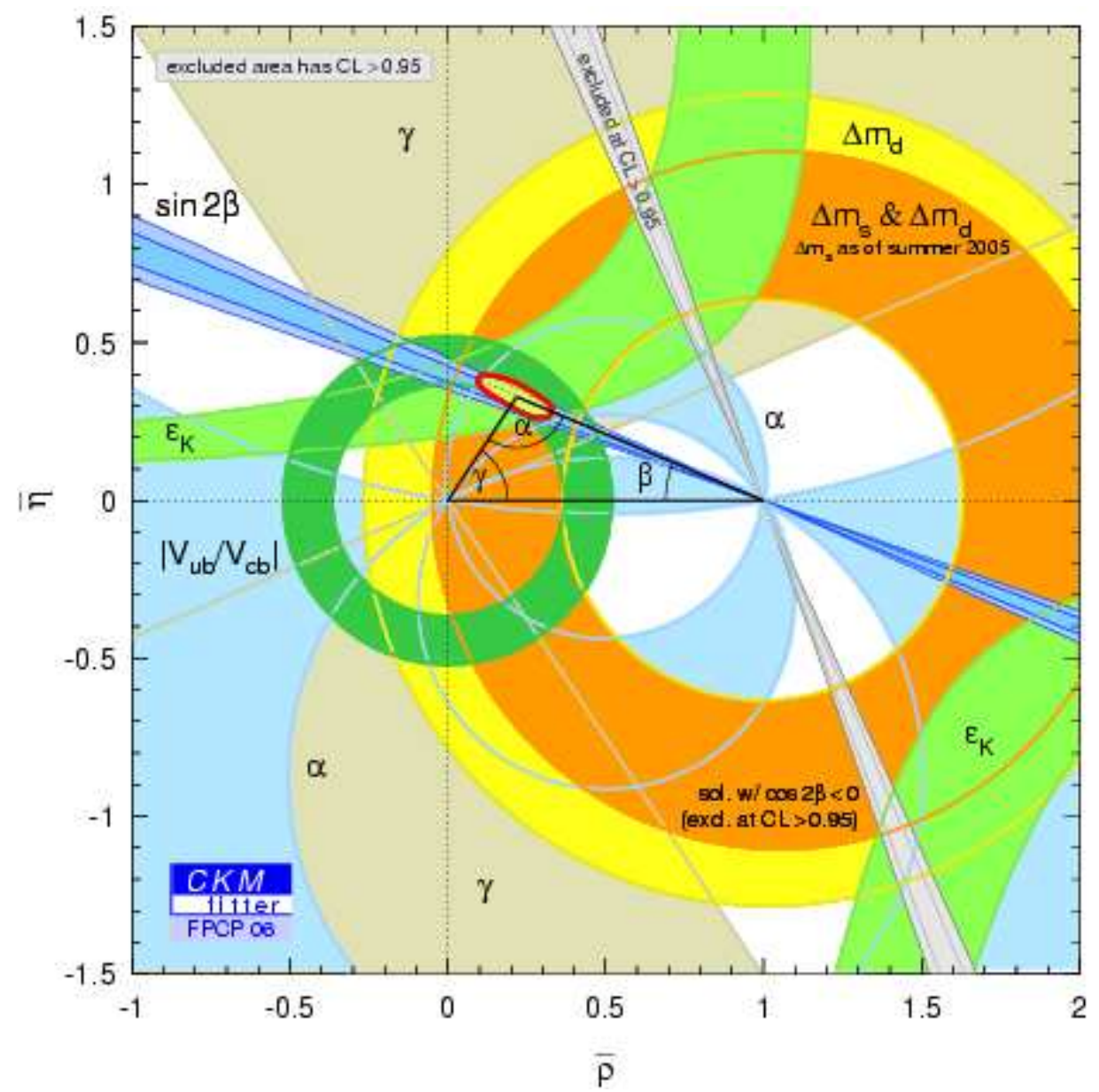

Figure 3.1. The understanding of the unitarity triangle prior to D-Zero's two-sided bound on $\Delta m_{s}$.

D-Zero has continued to work on studies of $B_{s}^{0}$ mixing, and has a preliminary measurement of $\Delta m_{s}$. D-Zero greatly improved on the original $B_{s}^{0}$ mixing publication by developing a number of additional analysis tools and improving existing ones. The 2006 mixing publication used only one $B_{s}^{0}$ decay channel - the same channel used in the analysis described in this thesis - and was done using the D-Zero RunIIa detector with about 


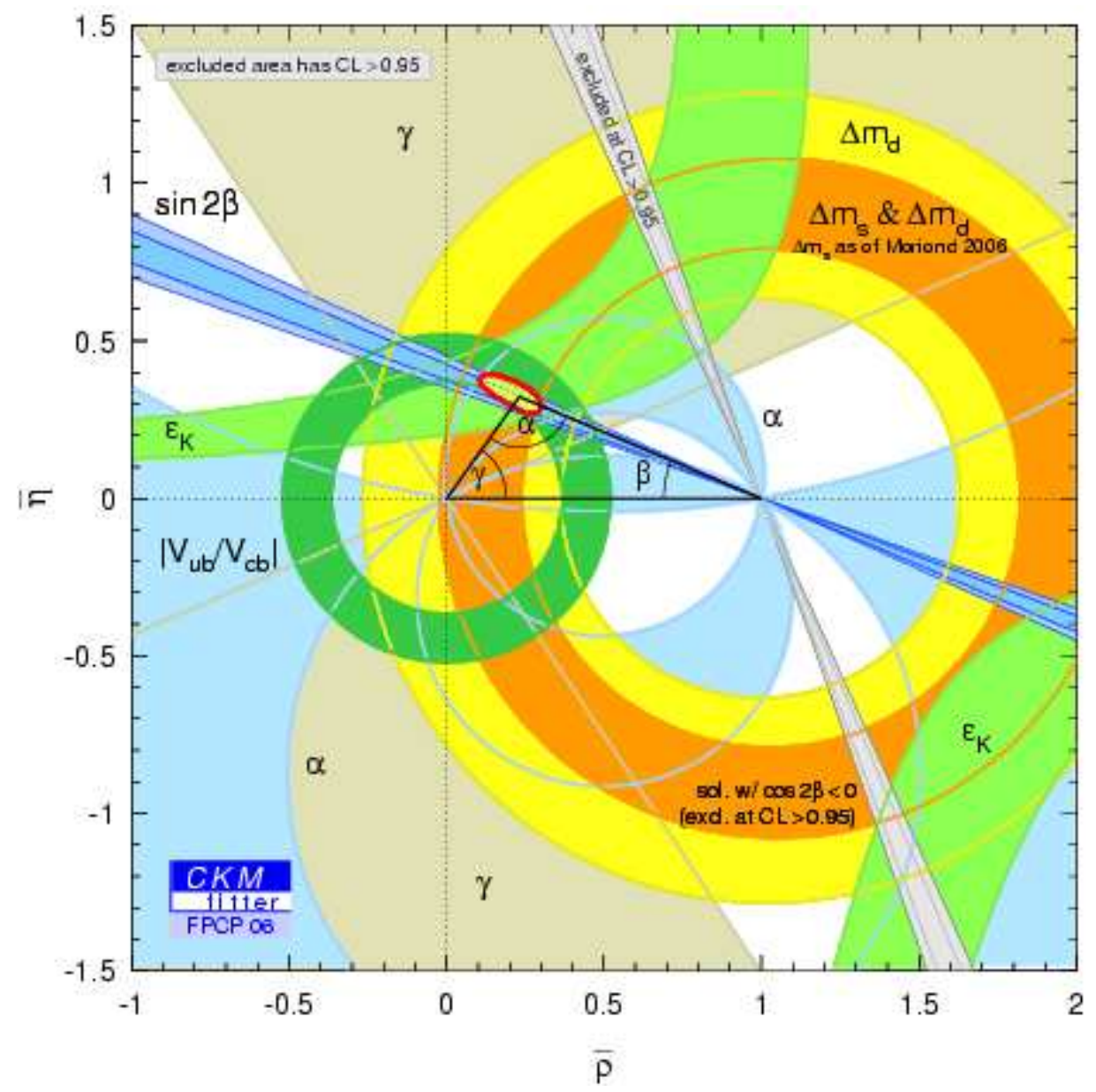

Figure 3.2. The understanding of the unitarity triangle after D-Zero's twosided bound on $\Delta m_{s}$ was included.

$1.0 \mathrm{fb}^{-1}$ of integrated luminosity. Shortly after the 2006 paper, D-Zero installed an additional layer of silicon called Layer $\varnothing$ very close to the beam pipe, improving the decay length resolution of the D-Zero detector. Layer $\varnothing$ and its readout electronics are discussed in greater detail in Section 2.2.2.1. The improvements to this analysis gained with the addition of Layer $\varnothing$ are discussed in Sections 3.5 and 3.6.4. Since the paper, D-Zero has worked to include an additional three semileptonic channels and one hadronic channel, 


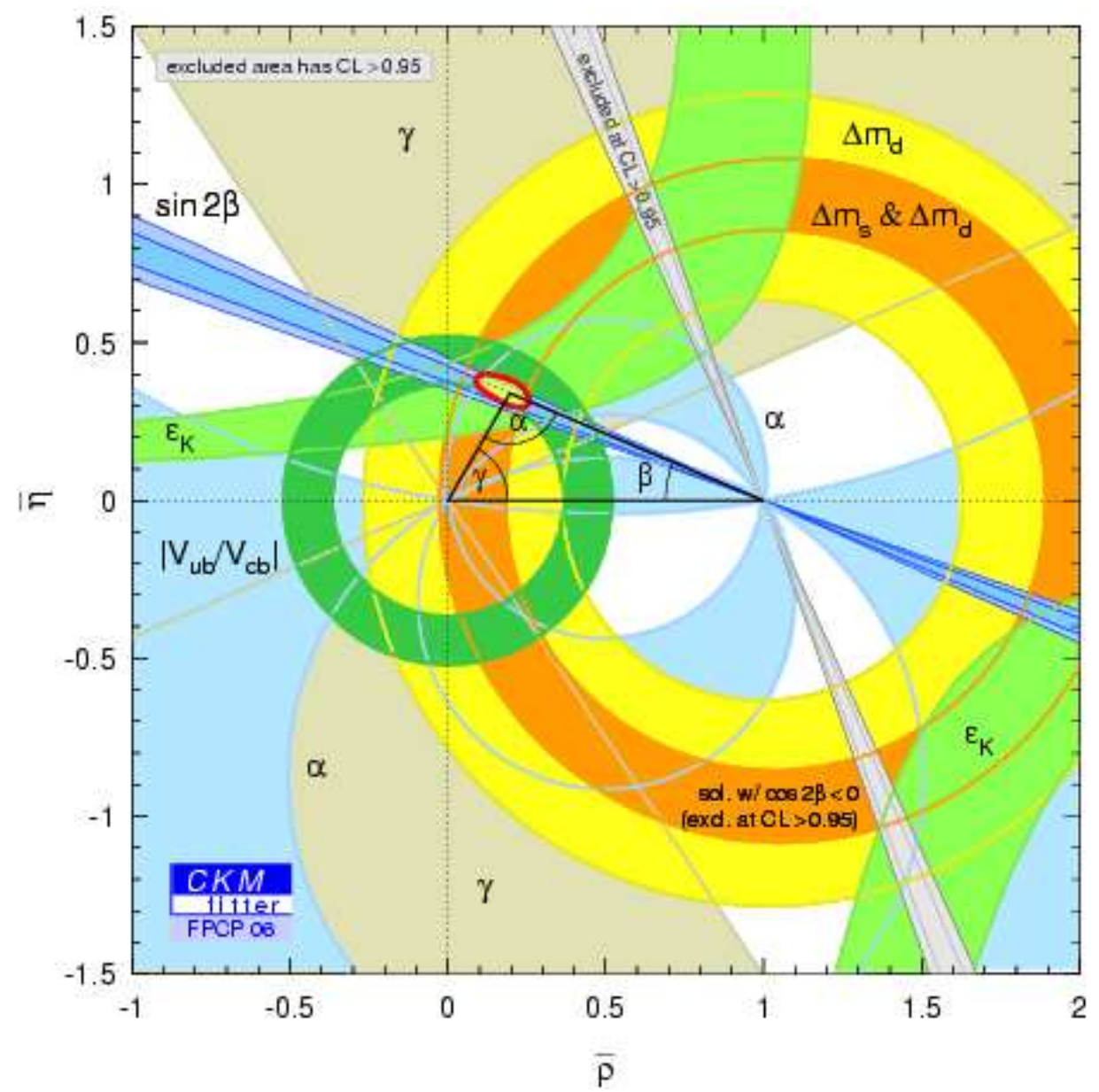

Figure 3.3. The understanding of the unitarity triangle after D-Zero's twosided bound on $\Delta m_{s}$ and CDF's discovery result were included.

greatly improving the sensitivity of the studies as well as the statistical significance. Additionally, the $B_{s}^{0}$ mixing group at D-Zero has made a number of offline improvements to the analyses. Flavor tagging, the ability to determine whether the $B_{s}^{0}$ was a $B_{s}^{0}$ or $\bar{B}_{s}^{0}$ at production and decay, was improved with the addition of event-charge tagging and same-side tagging. Same-side tagging uses information from the "side" of the event where the $B_{s}^{0}$ was produced to flavor-tag the event. Flavor tagging is discussed in detail in Section 3.3.1. The uncertainty on decay length resolution was improved by using an 
event-by-event scaling factor to correct the estimated uncertainty on the impact parameter; this is discussed in detail in Section 3.6.5, and improvements in analysis components due to the event-by-event scale factors are shown in Sections 3.5 and 4.1.2. Additionally, improvements were made to momentum scale factors called $K$ factors, which account for the missing neutrino or other unreconstructed particles in the event. Unreconstructed decay particles bias the $B_{s}^{0}$ momentum towards smaller values and $K$ factors provide the correction to this bias; $K$ factors are discussed in Section 3.6 .2 Finally, well over twice the integrated luminosity was used for this analysis, greatly increasing the statistics of the $B_{s}^{0}$ sample.

\subsection{Particle Reconstruction and Event Selection}

The selection of $B_{s}^{0} \rightarrow D_{s}^{-} \mu^{+} \nu X, D_{s}^{-} \rightarrow \phi \pi^{-}$events used a multi-step procedure. First, all tracks in a single event were clustered into jets using the DURHAM [47] clustering algorithm, using a $15 \mathrm{GeV} / c$ cutoff parameter. Muons with a track-match and $p_{T}>2 \mathrm{GeV} / c$ were selected, and the three tracks associated with the $K^{+}, K^{-}$, and $\pi^{-}$ had to come from the same jet as the selected muon. The kaons and pion had to pass transverse momentum cuts of $p_{T}>0.7 \mathrm{GeV} / c$ and $p_{T}>0.5 \mathrm{GeV} / c$, respectively. The tracks associated with the kaons were also required to have significant impact parameters with respect to the primary vertex (PV). The kaon and pion tracks had to form a vertex (the $D_{s}$ vertex), and the $D_{s}$ candidate had to form a vertex with the selected muon. Additionally, the $D_{s}$ candidate was required to be further from the primary vertex than the $B_{s}^{0}$ candidate, and both the $B_{s}^{0}$ and $D_{s}^{-}$momentum vectors had to reasonably point towards their respective vertices from the primary vertex. 
The final sample was selected using a likelihood ratio method based upon the following discriminating variables:

- Helicity angle, defined as the angle between the $D_{s}^{-}$and the $K^{+}$mesons in the $\left(K^{+}, K^{-}\right)$center-of-mass system;

- Isolation, computed as the ratio of the momentum of the $\mu^{+} D_{s}^{-}$system divided by the sum of the momentum of all charged particles in a cone $\Delta R=\sqrt{(\Delta \phi)^{2}+(\Delta \eta)^{2}}<0.5$, where $\Delta \eta$ and $\Delta \phi$ are the pseudo-rapidity and the azimuthal angle with respect to the $\mu^{+} D_{s}^{-}$direction. The $\mu^{+}, K^{+}, K^{-}$and $\pi^{-}$momenta are not included in the sum;

- $p_{T}\left(K^{+} K^{-}\right)$

- Invariant mass of the $\mu^{+} D_{s}^{-}$system, $m\left(\mu^{+} D_{s}^{-}\right)$;

- $\chi^{2}$ of the $D_{s}^{-}$vertex;

- Invariant mass of the $K^{+} K^{-}$system, $m\left(K^{+} K^{-}\right)$.

By definition, discriminating variables have different distributions for signal and background events. Using these discriminating variables, we construct probability density functions $(p d f \mathrm{~s})$ for signal $\left(f_{i}^{s}\left(x_{i}\right)\right)$ and background $\left(f_{i}^{b}\left(x_{i}\right)\right)$. Probability density functions describe probability distributions in terms of integrals, such that the integral over the whole probability distribution is one. The discriminating variables can have continuous or discrete distributions, depending on the variable. Using the probability density functions constructed for signal and background, a combined selection variable can be defined as:

$$
y=\prod_{i=1}^{n} y_{i} ; y_{i}=\frac{f_{i}^{b}\left(x_{i}\right)}{f_{i}^{s}\left(x_{i}\right)} .
$$


The probability density functions were determined using real data and are shown in Section 3.5. Additional details of the event selection are described in Reference [48].

\subsection{Flavor Tagging}

Flavor tagging determines whether one has reconstructed a $B_{s}^{0}$ or $\bar{B}_{s}^{0}$ meson. The flavor must be known at both production and decay to determine whether the $B_{s}^{0}$ mixed or not. Figure 3.4 shows an example of a $B_{s}^{0}$ candidate event. The center of the figure marks the $p \bar{p}$ collision point. The "side" of the event where the $B_{s}^{0}$ is produced is labeled as the "vertex side", to the right of the dashed line; this is also called the "reconstructed side" or the "same-side". The "opposite-side" of the event is on the left portion of the figure, where nearly always another $B$ meson or baryon is produced.

At D-Zero, there are two different methods used for determining the production flavor. One is called Opposite-Side Tagging, or OST, which uses information from the opposideside of the event illustrated in Figure 3.4. The other method is Same-Side Tagging, or SST, and uses information from the vertex side of the event. These are discussed in detail in Sections 3.3.1.1 and 3.3.1.2. Both taggers utilize a likelihood ratio method based on properties of the reconstructed $b$ hadron (SST) or the other $b$ hadron in the event (OST).

A single method is used for determining the decay flavor in semileptonic channels and is discussed in Section 3.3.2. The initial-state flavor-tagging is discussed first. 


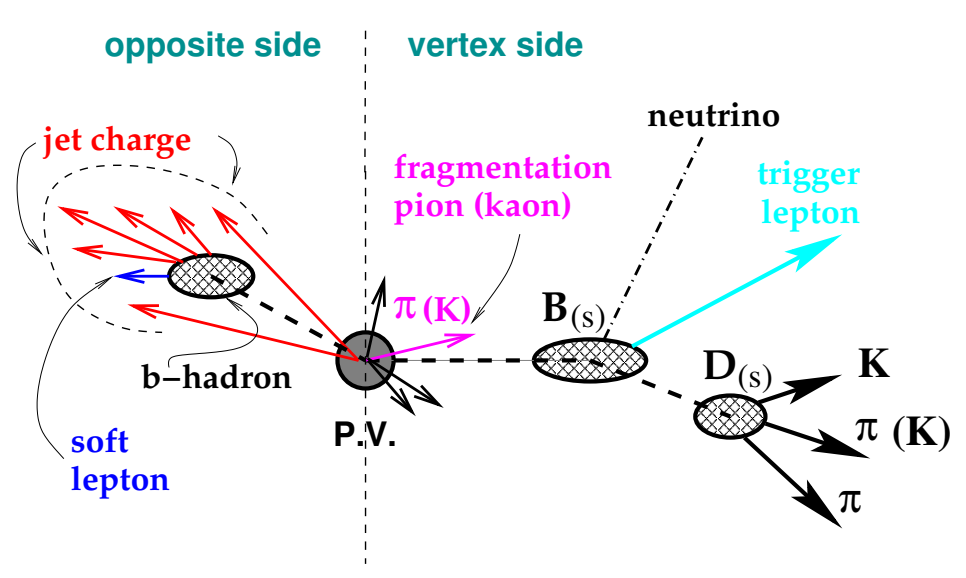

Figure 3.4. An example of an event with a $B_{s}^{0}$. Diagram courtesy of Sasha Rakitin.

\subsubsection{Initial-State Flavor Tagging}

For both OST and SST, the tagging information is based on probability density functions $(p d f \mathrm{~s})$ for $b$ and $\bar{b}$ quarks. The $p d f \mathrm{~s}$ are constructed from Monte-Carlo (MC) information. Monte-Carlo is a method of simulating physical systems, and is commonly used in particle physics to simulate particular decays or particle behaviors within a specific detector system. The MC used for the flavor-tagging was generated with Pythia [49] and EvtGen 50] 1 .

The $p d f \mathrm{~s}$ are constructed from the relevant variable for that tagger. These variables are described in the sections below. Once the $p d f \mathrm{~s}$ are constructed, the ratio of the functions for $b$ and $\bar{b}$ quarks is parameterized and defined as:

$$
y=\prod_{i=1}^{n} y_{i} ; y_{i}=\frac{f_{i}^{\bar{b}}\left(x_{i}\right)}{f_{i}^{b}\left(x_{i}\right)}
$$

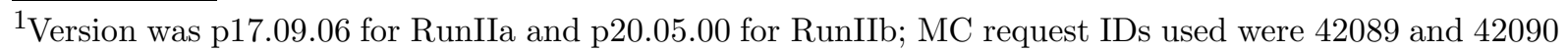
for RunIIa and 44162 through 44166 for RunIIb.
} 
where the $f_{i}^{b}\left(x_{i}\right)$ and $f_{i}^{\bar{b}}\left(x_{i}\right)$ are the $p d f_{\mathrm{s}}$ for each discriminating variable. It is more convenient to use a combined tagging variable in the analysis. This variable is defined as:

$$
d=\frac{1-y}{1+y}
$$

and is used to differentiate between $b$ and $\bar{b}$ quarks. The parameter $d$ varies between -1 and 1. An event with $d>0$ is tagged as $b$ quark and that with $d<0$ as a $\bar{b}$ quark, with larger $|d|$ values corresponding to higher tagging purities. $d$ can also be written as a function of the likelihood $(\mathcal{L})$ for the candidate to be a $B_{s}^{0}$ or $\bar{B}_{s}^{0}$ :

$$
d=\frac{\mathcal{L}\left(\bar{B}_{s}^{0}\right)-\mathcal{L}\left(B_{s}^{0}\right)}{\mathcal{L}\left(\bar{B}_{s}^{0}\right)+\mathcal{L}\left(B_{s}^{0}\right)}
$$

where $\mathcal{L}\left(B_{s}^{0}\right)$ is the likelihood for the event to be a $B_{s}^{0}$, and $\mathcal{L}\left(\bar{B}_{s}^{0}\right)$ is the likelihood for the event to be a $\bar{B}_{s}^{0}$. The likelihoods for initial-state flavor tagging are multi-dimensional, and are functions of the discriminating variables of the tagger; the different taggers are described in the following sections.

Additionally, $d$ is related to the dilution of the tagger, where dilution is defined as:

$$
\mathcal{D}=2 \eta-1, \eta=\frac{N_{\text {correct }}}{N_{\text {total }}}
$$

$\eta$ here is the purity of the sample, $N_{\text {correct }}$ is the number of events with correct flavor tag, and $N_{\text {total }}$ is the total number of events.

The dilution is plotted as a function of $|d|$ and fit with a functional form. The functional form is called the calibration curve, and the OST and SST each have their own 
calibration curves. The calibration curve is what is used as input when fitting, to determine the dilution of each event.

3.3.1.1. Opposite-Side Tagging. $b$-quarks are nearly always produced in back-to-back pairs at D-Zero. Therefore, when a $B_{s}^{0}$-meson is found, a $B$-meson or baryon is also produced on the opposite side of the detector. When the opposite-side $B$-particle decays, the charge of the decay products can be used to determine whether the particle included a $b$-quark or a $\bar{b}$-quark. If it consisted of a $b(\bar{b})$ quark, then the $B_{s}$ meson was a $B_{s}^{0}\left(\bar{B}_{s}^{0}\right)$, as the signal-side of the event must have had a $\bar{b}(b)$ quark. The Opposite-Side Tag (OST) takes advantage of this correlation. It uses the charge of the decay lepton or jet, or the sign of the total charge in a given cone around the event (the event charge).

When a muon can be reconstructed on the opposite side of the $B_{s}^{0}$ candidate, where "opposite side" is defined as $\cos \phi\left(\mathbf{p}_{\mu}, \mathbf{p}_{B}\right)<0.8$ with $\phi$ defined as the angle between the two momentum vectors, the muon jet charge is used as a discriminating variable for the OST. The muon jet charge is defined as: $Q_{J}^{\mu}=\frac{\sum_{i} q^{i} p_{T}^{i}}{\sum_{i} p_{T}^{T}}$, where the sum is taken over all charged particles on the opposite side, including the muon, in a cone of $\Delta R=\sqrt{(\Delta \phi)^{2}+(\Delta \eta)^{2}}<0.5$ around the muon direction. Some events do not have a reconstructed opposite-side muon, but do have a reconstructed electron available. In this case, the electron jet charge is used as the discriminating variable. Similar to the muon jet charge, the electron jet charge is defined as: $Q_{J}^{e}=\frac{\sum_{i} q^{i} p_{T}^{i}}{\sum_{i} p_{T}^{i}}$, where the sum is taken in the same way as in the muon case.

When neither a muon nor an electron is available on the opposite side, the event charge is used as the discriminating variable. The event charge is defined as: $Q_{E V}=\frac{\sum_{i} q^{i} p_{T}^{i}}{\sum_{i} p_{T}^{i}}$. The sum is taken over all charged particles having $0.5<p_{T}<50 \mathrm{GeV} / c$ and $\cos \phi\left(\mathbf{p}, \mathbf{p}_{B}\right)<0.8$. 
Although no optimization of the $p_{T}$ cut has been made, the upper-bound is intended to prevent a single high- $p_{T}$ track from solely determining the event charge.

The secondary vertex charge is also used as a discriminating variable when the secondary vertex $(\mathrm{SV})$ can be reconstructed. It is defined as: $Q_{S V}=\frac{\sum_{i}\left(q^{i} p_{L}^{i}\right)^{0.6}}{\sum_{i}\left(p_{L}^{i}\right)^{0.6}}$, where the sum was taken over all particles included in the secondary vertex and $p_{L}^{i}$ is the longitudinal momentum of a single particle, taken with respect to the sum of all momenta of the particles associated with the secondary vertex.

Opposite-side tagging can be used on different types of $B$ mesons and produce the same information about the $B_{s}^{0}$ candidate in question. The OST algorithm has been studied and calibrated using $B^{+}$and $B^{0}$ decays, on both data and MC samples. The $p d f \mathrm{~s}$ for opposite-side tagging were determined from real $B^{+} \rightarrow \mu^{+} \nu D^{0}$ events in which the $B$ flavor is given by the sign of the muon. When applied to $B_{d}^{0}$ events, fits to the asymmetry distribution show values for the $B_{d}^{0}$ oscillation frequency, $\Delta m_{d}$, to be consistent with the world average value [6]. See Fig. 3.5 for an example. More detailed information about
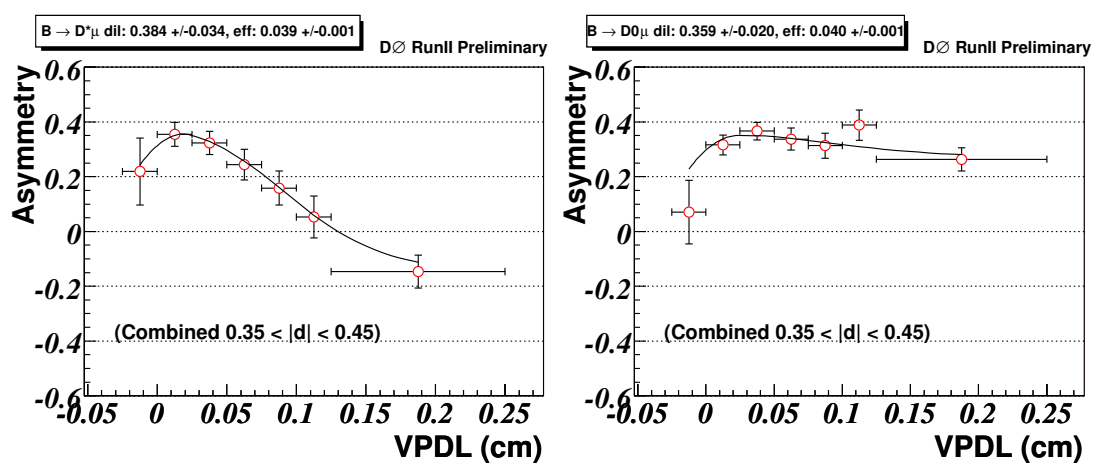

Figure 3.5. Flavor asymmetries for the $B \rightarrow \mu^{+} \nu D^{*-}$ and $B \rightarrow \mu^{+} \nu \bar{D}^{0}$ (predominantly unmixed) samples using the combined opposite side tagger in the tagging variable range $0.35<|d|<0.45$.

the OST can be found in Reference [51]. 
The calibration curve for the OST is given by:

$$
\mathcal{D}=0.457|d|+2.349|d|^{2}-2.498|d|^{3},|d|<0.6 ; \mathcal{D}=0.6,|d|>0.6 .
$$

This calibration curve is from a fit to the dilution versus $|d|$ curve shown in Figure 3.6 and was used in the previous analysis [17.

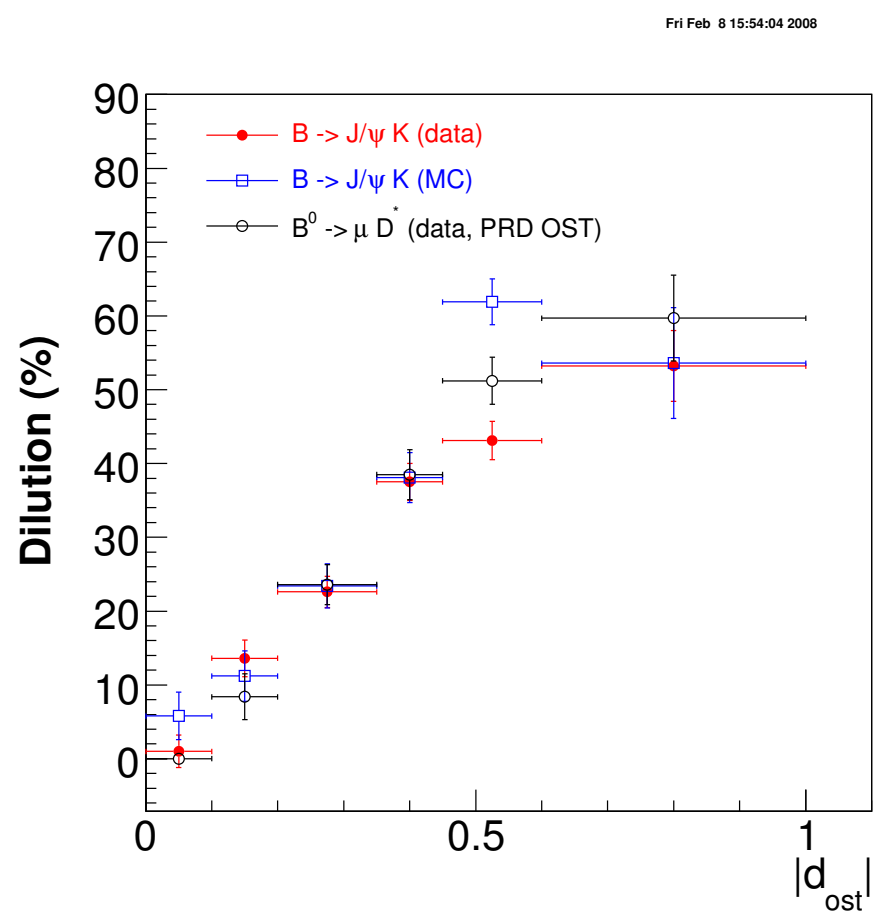

Figure 3.6. Dilution versus $|d|$ for the OST. The OST calibration curve is a fit to this distribution. 
3.3.1.2. Same-Side Tagging. Same-side tagging uses information on the same "side" of the event where the $B_{s}^{0}$ candidate is reconstructed. On the side of the event where the $B_{s}^{0}$ is produced, there are other particles produced at the same time from quark fragmentation. When a $B_{s}^{0}$ is produced, a fragmentation kaon is also produced, as shown in Figure 3.7. The charge of the kaon produced indicates the flavor of the $B_{s}^{0}$, where a $K^{+}$corresponds to a $B_{s}^{0}$ and a $K^{-}$corresponds to a $\overline{B_{s}^{0}}$. The same-side tagging algorithm takes advantage of this correlation between the charge of the fragmentation particle(s) and the flavor of the $B_{s}^{0}$. Charge correlations in quark fragmentation products were first observed by the OPAL experiment [52] in $e^{+} e^{-} \rightarrow Z^{0} \rightarrow b \bar{b}$ events.

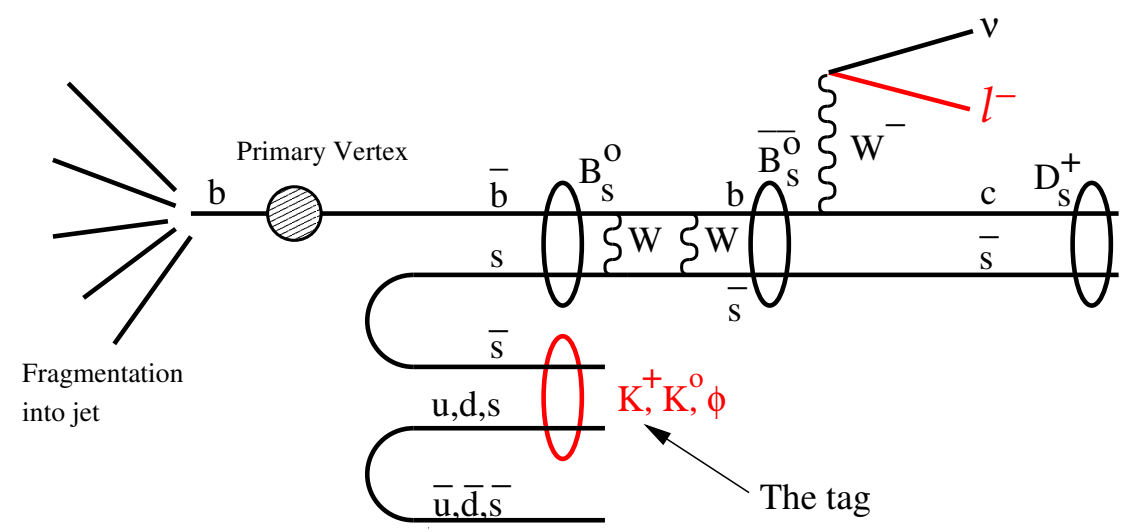

Figure 3.7. Illustration of the fragmentation products created when a $B_{s}^{0}$ is produced. The possible fragmentation products used for the flavor tagging are highlighted in red and labeled "The tag". Diagram courtesy of Sasha Rakitin.

There are two main types of same-side taggers: single-track taggers and many-track taggers. Single-track taggers are taggers using a single track which passes some kinematic requirements. Many-track taggers are taggers that use an averaged quantity from all of the tracks in the area surrounding the $B_{s}^{0}$. For the single-track tagger, the minimum distance between the momentum of the $B$ meson and the tag track provides the best tagging 
information. For the many-track tagger, the best tagging information is obtained from a $p_{T}$ weighted average of the charge of all the tracks in a cone defined as $\cos \alpha\left(\mathbf{p}, \mathbf{p}_{B}\right)>0.8$ around the $B_{s}^{0}$ momentum, where $\alpha$ is the angle between the track momentum vector and the $B_{s}^{0}$ momentum (defined as $\mathbf{p} \cdot \mathbf{p}_{B} /|p|\left|p_{B}\right|$ ).

These two taggers are combined using the same combination procedure used for the OST taggers [51, where the $p d f \mathrm{~s}$ are constructed from semileptonic $B_{s}^{0} \rightarrow D_{s}^{-}\left(\phi \pi^{-}\right) \mu^{+} \nu$ $\mathrm{MC}$ and $B_{s}^{0} \rightarrow J / \psi \phi \mathrm{MC}$. To ensure the validity of using MC samples for the tagging, a cross-check was done using a $B^{+} \rightarrow J / \psi K^{+}$data sample. Since charged $B$ mesons do not oscillate, the flavor of the $B_{s}^{0}$ inferred from the sign of the kaon was compared to the flavor given by the SST. A good agreement between the dilutions in data and MC was found. Figure 3.8 shows the data comparison with MC for the combined SST dilution in $B^{+} \rightarrow J / \psi K^{+}$decays. More detailed information about the SST can be found in Reference [53]. The calibration curves for the SST come from the dilution versus $|d|$ curves shown in Figure 3.9,

3.3.1.3. The Combined Tag. To utilize all of the tagging information available, the information from the OST, SST and event-charge tagger are combined into a single tagger, called the Combined Tag. The OST is the combination of the tag from Reference [51] and the event-charge tagger described earlier. The SST is the combination of the individual single-track and many-track same-side taggers.

The Combined Tag is created by combining the OST and SST using the same combination procedure as described in Reference [51]. The OST and SST pdfs are used to calculate a single value for $d$, using the equation $d=\frac{1-y_{S S T} \cdot y_{O S T}}{1+y_{S S T} \cdot y_{O S T}}$. The choice of tagger to be used is illustrated in the flowchart shown in Figure 3.10. 


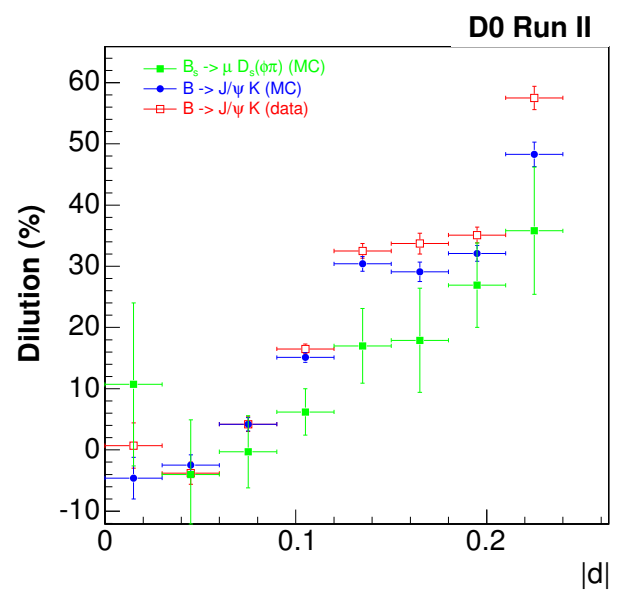

Figure 3.8. Dilution versus variable $|d|$ for the combined same-side tagger in the $B^{+} \rightarrow J / \psi K^{+}$sample (MC and data) and for $B_{s}^{0} \rightarrow \mu^{+} D_{s}^{-}\left(\phi \pi^{-}\right)$ (MC).

The dependence of dilution on $d$ for the combined SST+OST tag is shown in Figure 3.11, showing a linear relationship. For the combined tag, the tagging power $\epsilon D^{2}=$ $(4.49 \pm 0.88) \%$, which is larger than either the OST or SST tagging powers separately. The match between data and MC of dilution versus $d$ for the combined tag is shown in Figure 3.12 for $B^{ \pm} \rightarrow J / \psi K^{ \pm}$decays. The same dependence is shown for $B_{s}^{0} \rightarrow D_{s}^{-}(\phi \pi)$ $\mathrm{MC}$ is shown for comparison. As with the SST samples, we see a good agreement between data and $\mathrm{MC}$ for the $B^{ \pm} \rightarrow J / \psi K^{ \pm}$decays.

The calibration curve for the combined tag is given by:

$$
\begin{array}{r}
\mathcal{D}=78.95|d|+33.90|d|^{2},|d| \leq 0.6 \\
\mathcal{D}=60.65,|d|>0.6 .
\end{array}
$$

This calibration curve is from a fit to the dilution versus $|d|$ shown in Figure 3.13. 

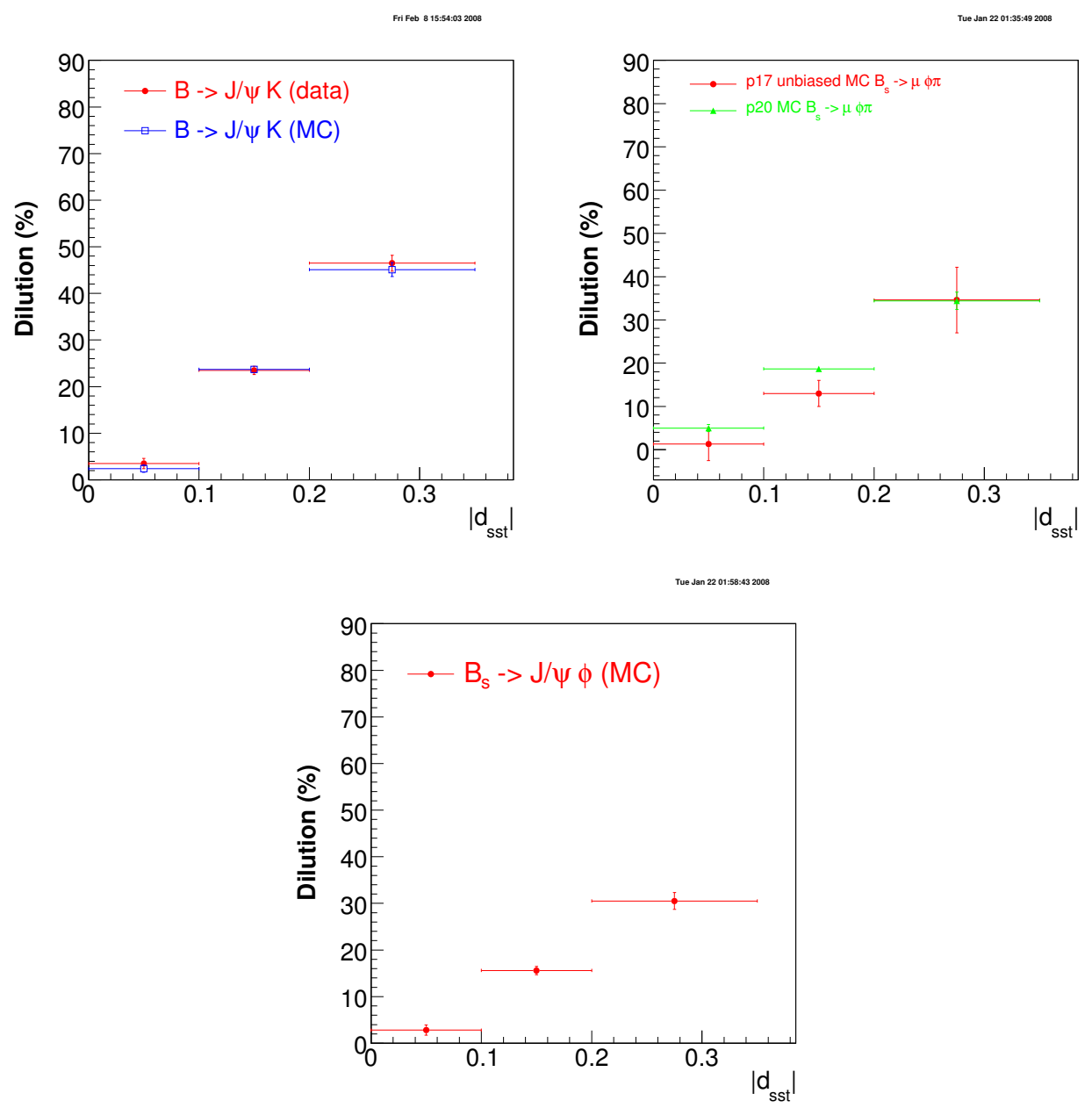

Figure 3.9. Dilution versus $|d|$ for the SST, for different data and MC samples.

3.3.1.4. Special Treatment of the $B_{u}$ Component Dilution. In addition to the inclusion of SST and event charge taggers for this analysis, we use a more correct description of the dilution for the $B^{+}$component. A $B^{+}$can decay semileptonically with the same final decay products as our signal; therefore, this $B^{+}$decay is treated as a signal component, as discussed in Section 3.5.1. The SST treats the sign of the dilution for the $B^{+}$component differently than the OST; for $B_{s}^{0}$ and $B_{d}^{0}$, the SST and OST give the same sign, but for $B_{u}$, they give opposite signs. This is illustrated in Figure 3.14, where 


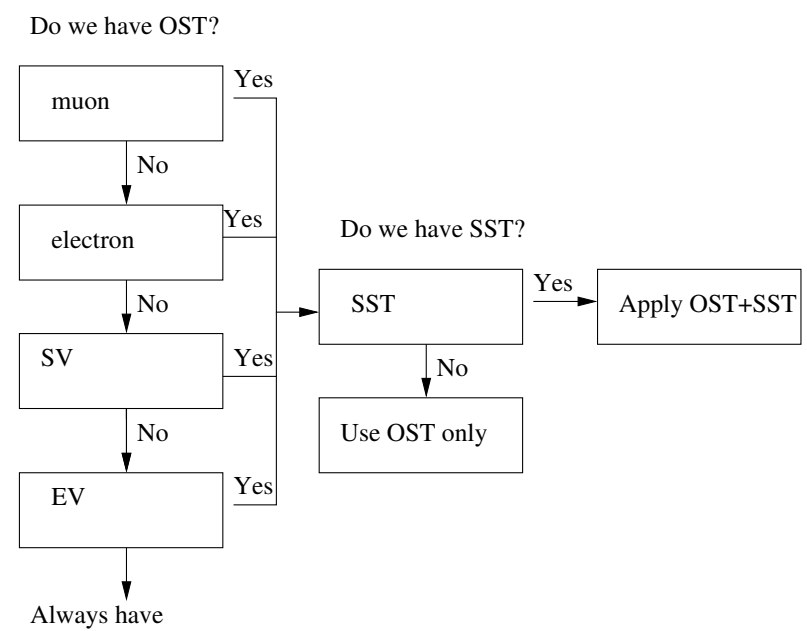

Figure 3.10. Flowchart of combination of SST + OST. "SV" stands for secondary vertex, and "EV" is event charge.

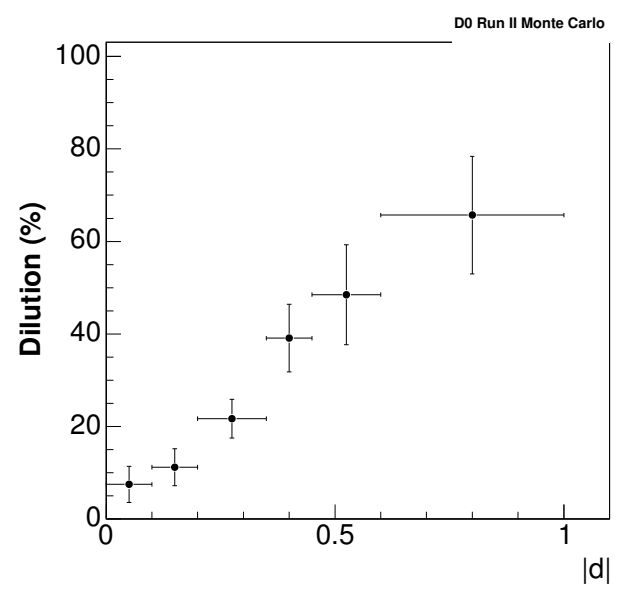

Figure 3.11. Dilution versus variable $|d|$ for the combined SST+OST tag in semileptonic $B_{s} \mathrm{MC}$.

the tag pion has a negative charge; in Figure 3.15, which illustrates a $B_{s}^{0}\left(\right.$ or $B_{d}^{0}$ ) event, the tag has a positive charge. In order to take this into account, the SST tagging variable for $B^{+}$must be redefined when the OST and SST are combined into a single tag. When the SST is redefined, the OST and SST components cancel each other out in the $B^{+}$component, resulting in a net dilution of about zero, as shown in Figure 3.15 For 


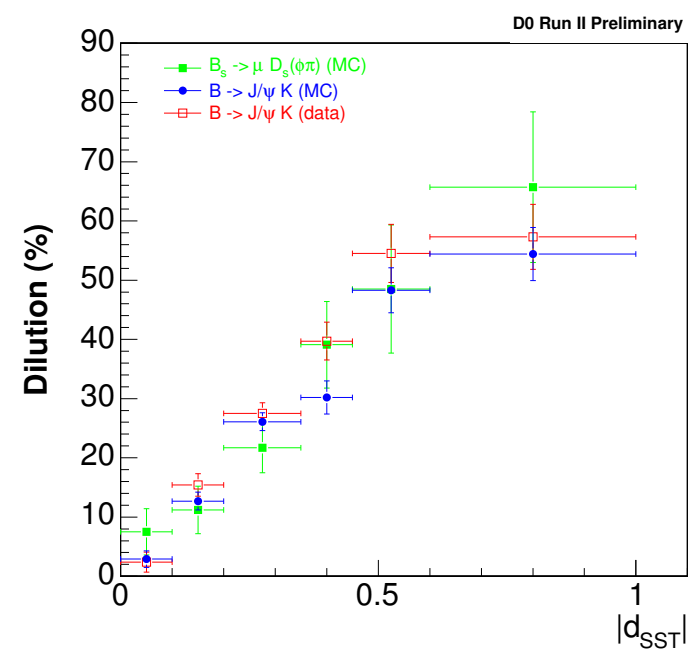

Figure 3.12. Dilution versus variable $|d|$ for combined same-side and opposite-side taggers for $B^{ \pm} \rightarrow J / \psi K^{ \pm}$events (data and Monte-Carlo) and for $B_{s}^{0} \rightarrow \mu^{+} D_{s}^{-}\left(\phi \pi^{-}\right)(\mathrm{MC})$.

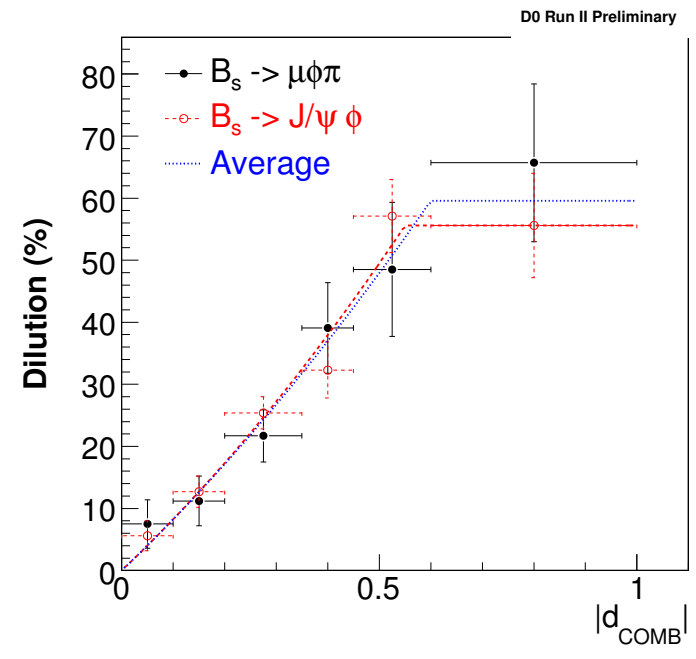

Figure 3.13. Dilution versus $|d|$ for the combined tag. The combined tag calibration curve is a fit to this distribution, shown in blue and labeled as "Average".

$|d|>0.45$ we essentially have only OST, which gives positive dilution. To ensure we do not use a negative net dilution for $|d| \leq 0.45$, we set the dilution to zero for $|d| \leq 0.45$. 
The complete equation for the $B^{+}$calibration is given by:

$$
\begin{array}{r}
\mathcal{D}=43.93|d|,|d| \geq 0.45 \\
\mathcal{D}=0,|d|<0.45 .
\end{array}
$$

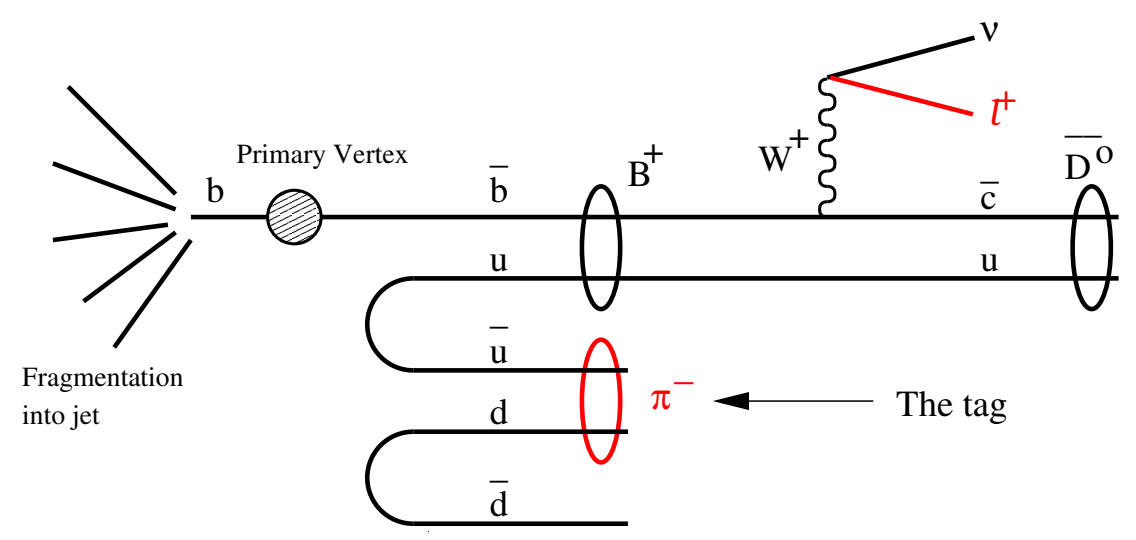

Figure 3.14. Illustration of the fragmentation products created when a $B_{u}$ is produced. The fragmentation pion used as the tag is highlighted in red and labeled "The tag". Note that the charge of the pion is opposite the tag particle for a $B_{s}^{0}$ or $B_{d}^{0}$, indicating that the SST is defined differently for $B^{+}$ decays. Diagram courtesy of Sasha Rakitin. 


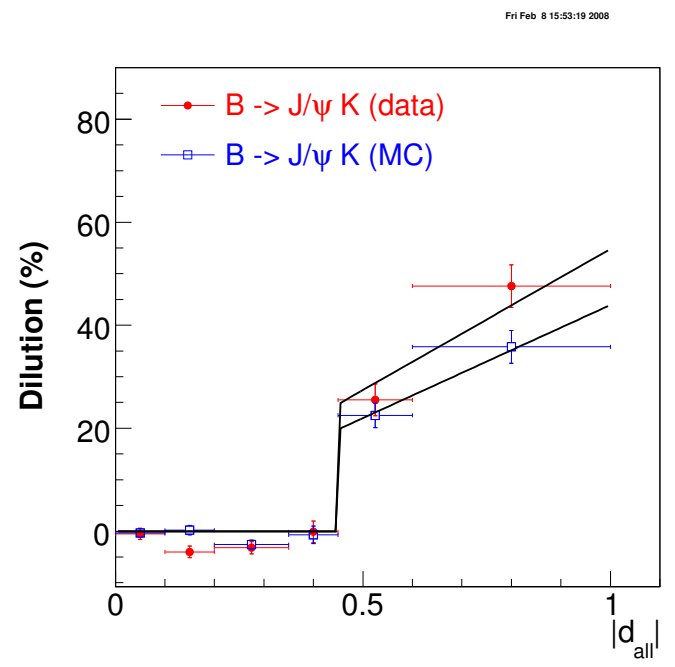

Figure 3.15. Dilution versus variable $|d|$ for $B^{ \pm} \rightarrow J / \psi K^{ \pm}$events (data and $\mathrm{MC}$ ), illustrating the calibration curve for the $B^{+}$component. The fit to the MC sample is used as the default $B^{+}$calibration curve. 


\subsubsection{Flavor Tagging at Decay}

The flavor of the $B_{s}^{0}$ at decay is determined by the charge of the decay lepton in semileptonic decays. For the $B_{s}^{0} \rightarrow D_{s}^{-} \mu^{+} \nu X$ channel, the flavor at decay is determined by the charge of the decay muon. A $\mu^{+}$indicates the meson was a $B_{s}^{0}$ at decay, while a $\mu^{-}$as the decay lepton indicates the meson was a $\bar{B}_{s}^{0}$ at decay. There are, however, $B_{s}^{0}$ decays where a $B_{s}^{0}$ can decay to a $\mu^{-}$(and a $\bar{B}_{s}^{0}$ to a $\mu^{+}$), and these are discussed in Section 3.5.1

\subsection{Mass Fitting Procedure}

To fit the mass of our tagged and untagged samples of $D_{s}^{-}$candidates, a double Gaussian distribution is used to describe the $D_{s}^{ \pm} \rightarrow \phi \pi$ decay, a single Gaussian distribution is used to describe the $D^{ \pm} \rightarrow \phi \pi$ decay and the background is modeled by an exponential (see Fig. 3.16 and Fig. 3.18). The contribution of the reflection $\mu D^{ \pm}(\rightarrow K \pi \pi)$, where the pion is misidentified as a kaon, is not fit from the mass distribution; this contribution is discussed in the next section. The corresponding fit to the tagged sample is shown in Fig. [3.17 and Fig. 3.19] The fit parameters are defined as:

- $p 0$ - number of events in the $D_{s}$ peak (right-side peak) divided by 100 ,

- $p 1$ - mean of $D_{s}$ peak,

- $p 2$ - sigma of first Gaussian fit to $D_{s}$ peak,

- $p 3$ - number of events in the $D^{ \pm}$peak (left-side peak) divided by 100 ,

- $p 4$ - mean of $D^{ \pm}$peak,

- $p 5$ - sigma of $D^{ \pm}$peak,

- $p 6$ - coefficient of the exponential background: $p 6 \cdot e^{p 7 \cdot x}$,

- $p 7$ - slope of the exponential background, 
- $p 8$ - sigma of second Gaussian fit to $D_{s}$ peak,

- $p 9$ - fraction of $D_{s}$ peak fitted with first Gaussian.

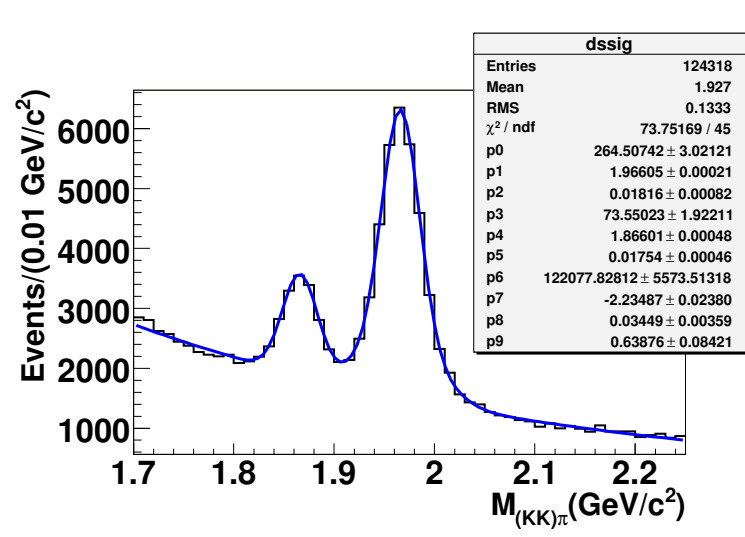

Figure 3.16. All $B_{s}$ candidates for the untagged sample using RunIIa data.

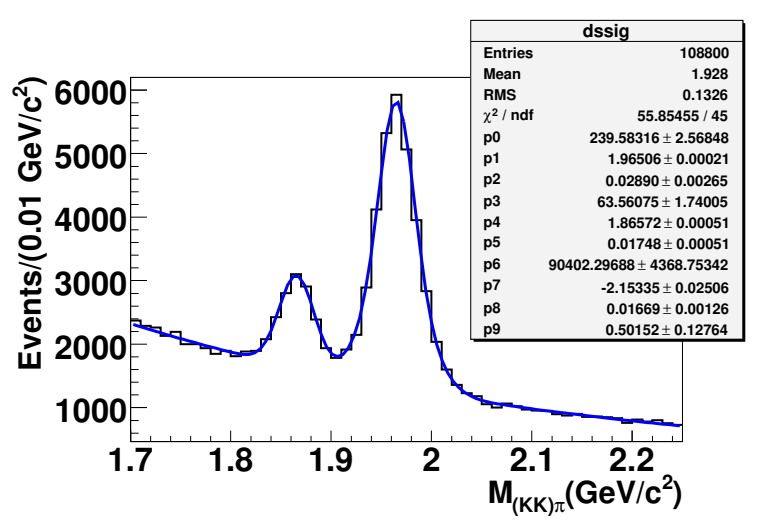

Figure 3.18. All $B_{s}$ candidates for the untagged sample using RunIIb data.

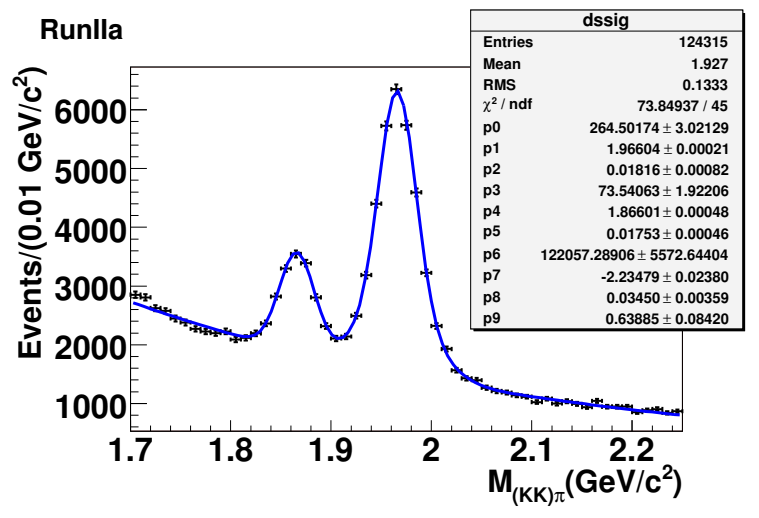

Figure 3.17. All $B_{s}$ candidates for the tagged sample using RunIIa data and the combined tag.

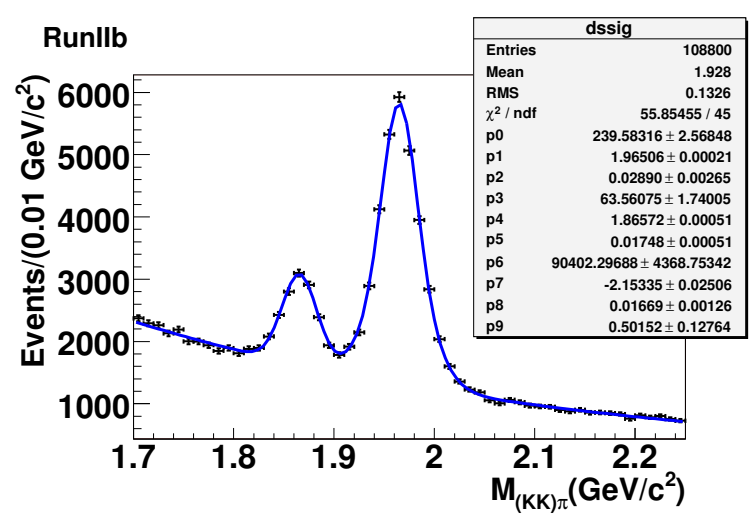

Figure 3.19. All $B_{s}$ candidates for the tagged sample using RunIIb data and the combined tag.

A comparison of the tagged and untagged samples shows that the tagging procedure does not significantly change the $D_{s}$ peak parameters. This was true for the previous analysis [17] as well, which confirmed this by using MC distributions for the signal events. 
Additionally, because the event charge flavor tagger has a nearly $100 \%$ efficiency, the difference in the mass fit parameters for the untagged and tagged samples is minimal. The untagged mass plots are shown for illustrative purposes only, as the tagged mass plots are of most interest for the analysis.

\subsection{Unbinned Likelihood Fit Method}

We use an unbinned likelihood fit method for the $B_{s}^{0}$ mixing analysis. To start, we define the variables used, setting $L_{x y}$ as the transverse decay length of the $B_{s}^{0}$, defined as the distance in the axial plane between the primary vertex and the $\mu^{+} D_{s}^{-}$vertex. A detailed description of the vertexing algorithm is given in Reference [54]. We label $P_{T}^{\mu^{+} D_{s}^{-}}$as the $B_{s}^{0}$ transverse momentum, which we define as the vector sum of the muon and $D_{s}^{-}$transverse momenta. Finally, we define the measured visible proper decay length (VPDL) as:

$$
V P D L=x^{M}=\left(\mathbf{L}_{\mathbf{x y}} \cdot \mathbf{P}_{\mathbf{x y}}^{\mu \mathbf{D}_{\mathbf{s}}}\right) /\left(P_{T}^{\mu D_{s}}\right)^{2} \cdot M_{B_{s}} .
$$

We use all events with $1.72<M_{(K K) \pi}<2.22 \mathrm{GeV} / c^{2}$ in the unbinned likelihood fitting procedure.

The likelihood for an event to arise from a specific source in the sample depends on the $x^{M}$, its error $\left(\sigma_{x^{M}}\right)$, the mass of the $D_{s}^{-}$meson candidate $(m)$ and the predicted dilution $\left(d_{p r}\right)$, where all of these quantities are known on an event-by-event basis. The $p d f$ of each source can be expressed by the following formula:

$$
f_{i}=P_{i}^{x^{M}}\left(x^{M}, \sigma_{x^{M}}, d_{p r}\right) P_{i}^{\sigma_{x}^{M}} P_{i}^{m} P_{i}^{d_{p r}} .
$$

The functions $P_{i}^{x^{M}}\left(x^{M}, \sigma_{x^{M}}, d_{p r}\right), P_{i}^{\sigma_{x} M}, P_{i}^{m}$, and $P_{i}^{d_{p r}}$ will be defined later. 
We considered the following sources:

- $\mu D_{s}(\rightarrow \phi \pi)$ signal with fraction $F r_{\mu D_{s}}$,

- $\mu D^{ \pm}(\rightarrow \phi \pi)$ signal with fraction $F r_{\mu D^{ \pm}}$,

- $\mu D^{ \pm}(\rightarrow K \pi \pi)$ reflection with fraction $F r_{\mu D_{\text {refl }}^{ \pm}}$, and

- combinatorial background with fraction $\left(1-F r_{\mu D_{s}}-F r_{\mu D^{ \pm}}-F r_{\mu D_{r e f l}^{ \pm}}\right)$.

The fractions $F r_{\mu D_{s}}$ and $F r_{\mu D^{ \pm}}$were determined from the mass fit (see Figs. 3.17and 3.19). The total $p d f$ for a $B$ candidate has the form:

$$
F_{n}=F r_{\mu D_{s}} f_{\mu D_{s}}+F r_{\mu D^{ \pm}} f_{\mu D^{ \pm}}+F r_{\mu D_{r e f l}^{ \pm}} f_{\mu D_{r e f l}^{ \pm}}+\left(1-F r_{\mu D_{s}}-F r_{\mu D^{ \pm}}-F r_{\mu D_{r e f l}^{ \pm}}\right) f_{b k g}
$$

The following form is minimized using the MINUIT program:

$$
\mathcal{L}=-2 \sum_{n=1}^{N_{\text {candidates }}} \ln F_{n}
$$

The $p d f s$ for the VPDL error (Figs. 3.20, 3.21, and 3.22), mass (Figs. 3.17 and 3.19) and dilution (Figs. 3.23 and 3.24 ) are taken from experimental data and are normalized when used in the fit; additional plots of the VPDL pdfs are shown in Appendix B to better illustrate the differences in the distributions. The signal $p d f \mathrm{~s}$ are also used for the $\mu D^{ \pm}(\rightarrow \phi \pi)$ signal and the $\mu D^{ \pm}(\rightarrow K \pi \pi)$ reflection. The mass $p d f$ for the $\mu D^{ \pm}(\rightarrow K \pi \pi)$ reflection was taken from a previous study [55] as an input to the fit and was given by a Gaussian centered at $2.036 \mathrm{GeV} / \mathrm{c}^{2}$ with width $0.0262 \mathrm{GeV} / \mathrm{c}^{2}$. The fraction of the reflection was set at the $0.6 \%$ level from the $\mu^{+} D_{s}^{-}$signal. 

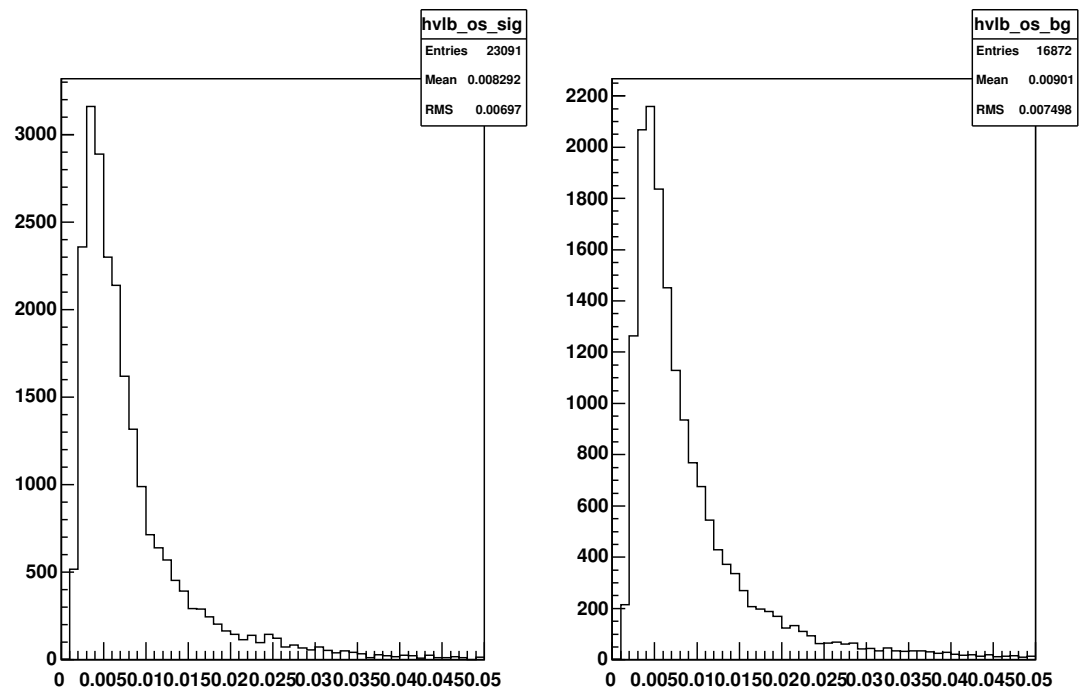

Figure 3.20. Distributions of VPDL errors for signal (left) and combinatorial background (right) in RunIIa data.
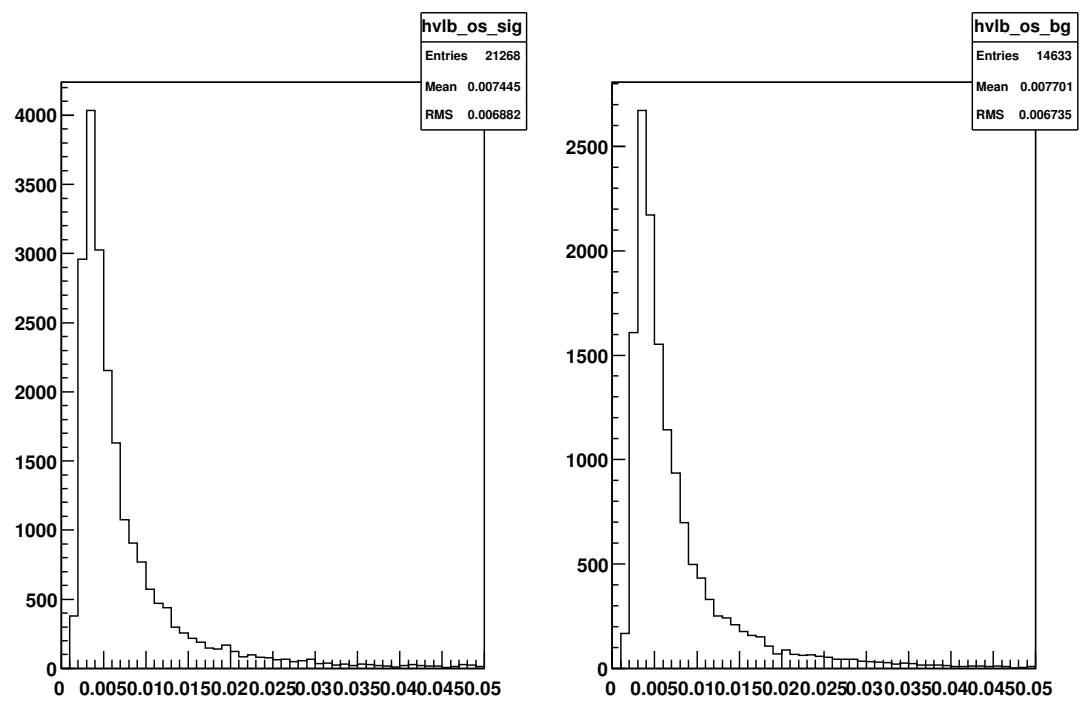

Figure 3.21. Distributions of VPDL errors for signal (left) and combinatorial background (right) in RunIIb data. 

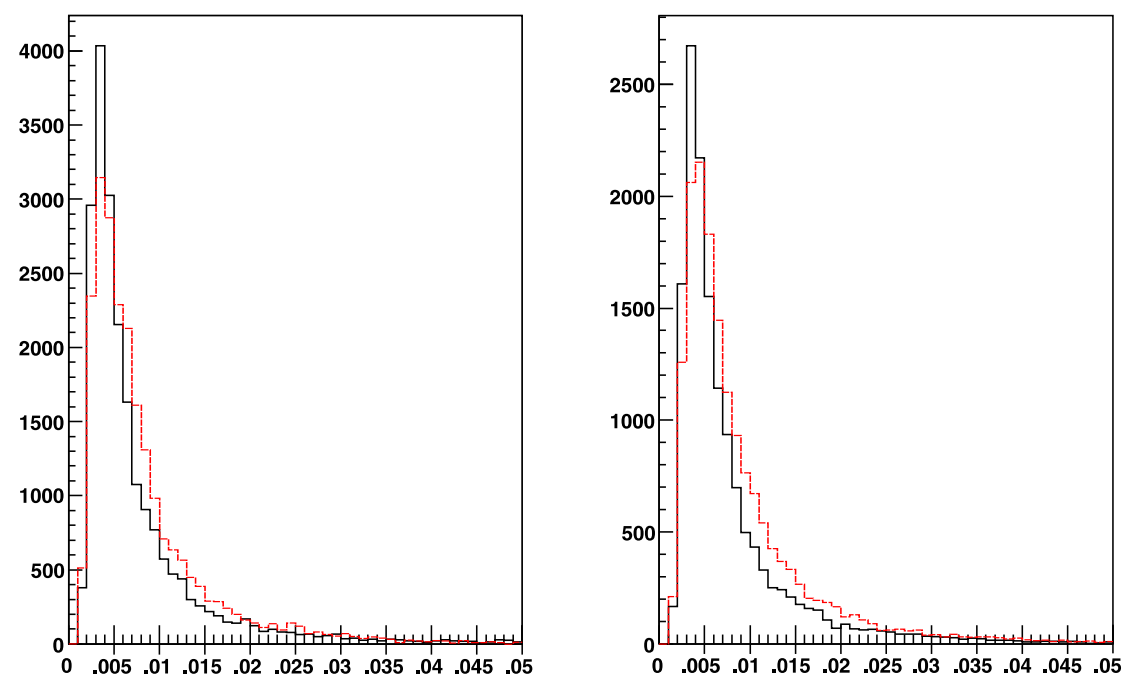

Figure 3.22. Distributions of VPDL errors for signal (left) and combinatorial background (right). RunIla data is indicated by the red dashed line, while RunIIb is the black line; both have the event-by-event $S F$ s for their run period applied.
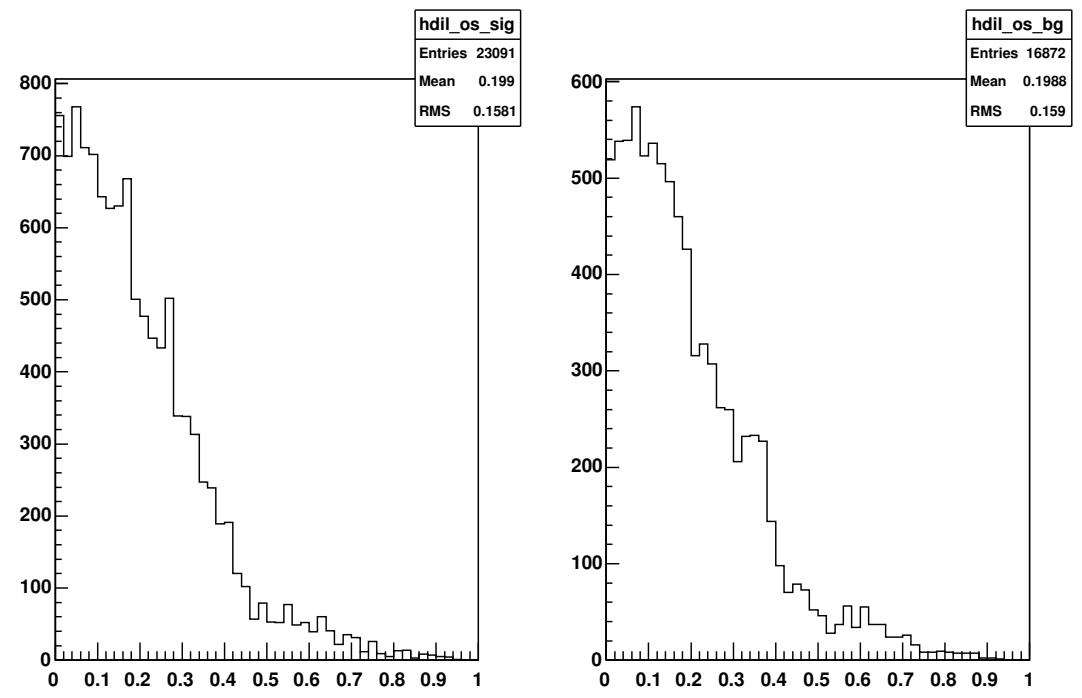

Figure 3.23. Distributions of predicted dilution for signal (left) and combinatorial background (right) in RunIIa data. 

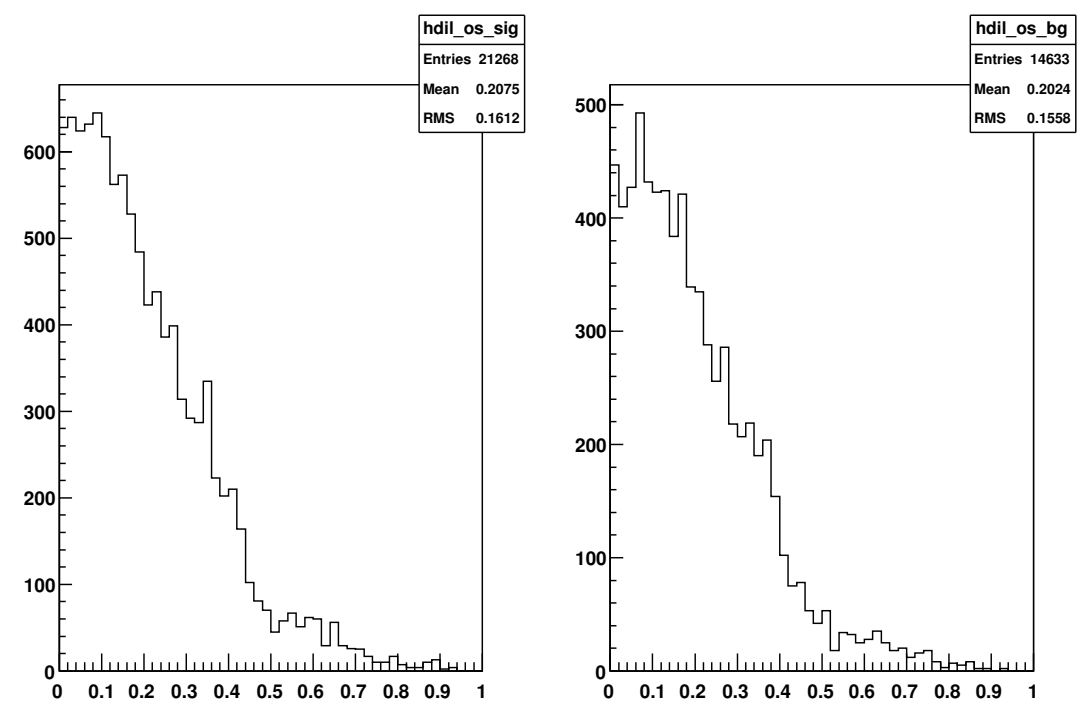

Figure 3.24. Distributions of predicted dilution for signal (left) and combinatorial background (right) in RunIIb data.

\subsection{1. $p d f$ for $\mu^{+} D_{s}^{-}$Signal}

The $\mu^{+} D_{s}^{-}$sample is composed mostly of $B_{s}$ mesons with some contributions from $B_{u}$ and $B_{d}$ mesons. Different species of $B$ mesons behave differently with respect to oscillations. Neutral $B_{d}$ and $B_{s}$ mesons do oscillate (with different frequencies) while charged $\mathrm{B}_{u}$ mesons do not, as that would violate charge conservation. The possible contributions of $b$ baryons in the sample are expected to be small 2 and so are neglected.

${ }^{2}$ This can be argued from the small statistics available for analyses such as the $\Lambda_{b}$ lifetime [56], [57] and the observation of the $\Xi_{b}[\mathbf{5 8}$. 
For a given type of $B$ hadron (i.e., $d, u, s$ ), the distribution of the visible proper decay length $x$ is given by:

$$
\begin{aligned}
& p_{s}^{n o s}\left(x, K, d_{p r}\right)=\frac{K}{c \tau_{B_{s}}} \exp \left(-\frac{K x}{c \tau_{B_{s}}}\right) \cdot 0.5 \cdot\left(1+\mathcal{D}\left(d_{p r}\right) \cos \left(\Delta m_{s} \cdot K x / c\right)\right)(3.1 \\
& p_{s}^{o s c}\left(x, K, d_{p r}\right)=\frac{K}{c \tau_{B_{s}}} \exp \left(-\frac{K x}{c \tau_{B_{s}}}\right) \cdot 0.5 \cdot\left(1-\mathcal{D}\left(d_{p r}\right) \cos \left(\Delta m_{s} \cdot K x / c\right)\right)(3.1 \\
& p_{D s D s}^{o s c}(x, K)=\frac{K}{c \tau_{B_{s}}} \exp \left(-\frac{K x}{c \tau_{B_{s}}}\right) \cdot 0.5 \\
& p_{D s D s}^{\text {nos }}(x, K)=\frac{K}{c \tau_{B_{s}}} \exp \left(-\frac{K x}{c \tau_{B_{s}}}\right) \cdot 0.5 \\
& p_{u}^{\text {nos }}\left(x, K, d_{p r}\right)=\frac{K}{c \tau_{B_{u}}} \exp \left(-\frac{K x}{c \tau_{B_{u}}}\right) \cdot 0.5 \cdot\left(1-\mathcal{D}_{B_{u}}\left(d_{p r}\right)\right) \\
& p_{u}^{o s c}\left(x, K, d_{p r}\right)=\frac{K}{c \tau_{B_{u}}} \exp \left(-\frac{K x}{c \tau_{B_{u}}}\right) \cdot 0.5 \cdot\left(1+\mathcal{D}_{B_{u}}\left(d_{p r}\right)\right) \\
& p_{d}^{n o s}\left(x, K, d_{p r}\right)=\frac{K}{c \tau_{B_{d}}} \exp \left(-\frac{K x}{c \tau_{B_{d}}}\right) \cdot 0.5 \cdot\left(1-\mathcal{D}\left(d_{p r}\right) \cos \left(\Delta m_{d} \cdot K x / c\right)\right) \\
& p_{d}^{o s c}\left(x, K, d_{p r}\right)=\frac{K}{c \tau_{B_{d}}} \exp \left(-\frac{K x}{c \tau_{B_{d}}}\right) \cdot 0.5 \cdot\left(1+\mathcal{D}\left(d_{p r}\right) \cos \left(\Delta m_{d} \cdot K x / c\right)\right)(3.2 \\
& p_{\bar{B}_{s}^{0} \rightarrow D_{s}^{-} D}^{n o s}\left(x, K, d_{p r}\right)=\frac{K}{c \tau_{B_{s}}} \exp \left(-\frac{K x}{c \tau_{B_{s}}}\right) \cdot 0.5 \cdot\left(1-\mathcal{D}\left(d_{p r}\right) \cos \left(\Delta m_{s} \cdot K x / c\right)\right)( \\
& p_{B_{s}^{0} \rightarrow D_{s}^{-} D}^{o s c}\left(x, K, d_{p r}\right)=\frac{K}{c \tau_{B_{s}}} \exp \left(-\frac{K x}{c \tau_{B_{s}}}\right) \cdot 0.5 \cdot\left(1+\mathcal{D}\left(d_{p r}\right) \cos \left(\Delta m_{s} \cdot K x / c\right)\right)( \\
& p_{B_{s}^{0} \rightarrow D_{s}^{-} D}^{o s c}\left(x, K, d_{p r}\right)=\frac{K}{c \tau_{B_{s}}} \exp \left(-\frac{K x}{c \tau_{B_{s}}}\right) \cdot 0.5 \cdot\left(1-\mathcal{D}\left(d_{p r}\right) \cos \left(\Delta m_{s} \cdot K x / c\right)\right)( \\
& p_{B_{s}^{0} \rightarrow D_{s}^{-} D}^{\text {nos }}\left(x, K, d_{p r}\right)=\frac{K}{c \tau_{B_{s}}} \exp \left(-\frac{K x}{c \tau_{B_{s}}}\right) \cdot 0.5 \cdot\left(1+\mathcal{D}\left(d_{p r}\right) \cos \left(\Delta m_{s} \cdot K x / c\right)\right)(3.2
\end{aligned}
$$


where

$$
\begin{gathered}
K=P_{T}^{\mu^{+} D_{s}^{-}} / P_{T}^{B} \\
\mathcal{D}\left(d_{p r}\right)=78.95 \cdot\left|d_{p r}\right|+33.90 \cdot|d|^{2},|d| \leq 0.6 \\
\mathcal{D}\left(d_{p r}\right)=60.65,|d|>0.6 ; \\
\mathcal{D}_{B_{u}}\left(d_{p r}\right)=43.93 \cdot|d|,|d| \geq 0.45 \\
\mathcal{D}_{B_{u}}\left(d_{p r}\right)=0,|d|<0.45
\end{gathered}
$$

(see Figs. 3.13 and 3.15 and Eqns. 3.7 and 3.8).

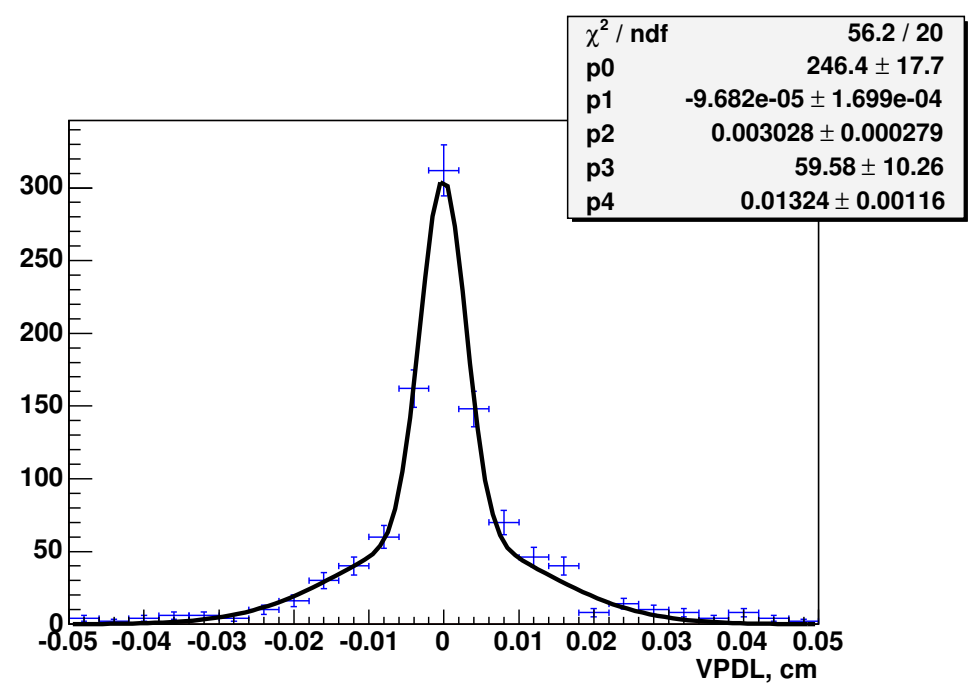

Figure 3.25. VPDL distribution for the peaking background.

Here $\tau$ is the lifetime of the $B$ hadron and $K$ is the $K$ factor, which reflects the difference between the observable and true momenta of the $B$-hadron (see Section 3.6 .2 for a detailed description of $K$ factors). Note that there is a sign swap in Equations 3.17 3.22 with respect to 3.13 and 3.14 due to anti-correlation of charge for muons from $B \rightarrow D D_{s}$; $D \rightarrow \mu X$ processes. 
The translation to the measured VPDL, $x^{M}$, is achieved by a convolution of the $K$ factors and resolution functions as specified below.

$$
\begin{array}{r}
P_{(d, u, s), j}^{o s c, n o s}\left(x^{M}, \sigma_{x^{M}}, d_{p r}\right)=\int_{K_{\min }}^{K_{\max }} d K D_{j}(K) \cdot \frac{E f f_{j}\left(x^{M}\right)}{N_{j}\left(K, \sigma_{x^{M}}, d_{p r}\right)} \\
\int_{0}^{\infty} d x G\left(x-x^{M}, \sigma_{x^{M}}\right) \cdot p_{(d, u, s), j}^{o s c, n o s}\left(x, K, d_{p r}\right) . \\
\text { Here } G\left(x-x^{M}, \sigma_{x^{M}}\right)=\frac{1}{\sqrt{2 \pi} \sigma_{x^{M}}} \exp \left(-\frac{\left(x-x^{M}\right)^{2}}{2 \sigma_{x^{M}}^{2}}\right)
\end{array}
$$

is the detector resolution of the VPDL and $E f f_{j}(x)$ is the reconstruction efficiency for a given decay channel $j$ of this type of $B$ meson as a function of VPDL. The function $D_{j}(K)$ gives the normalized distribution of the $K$-factor in a given channel $j$. The normalization factor $N_{j}$ is calculated by integration over the entire VPDL region:

$$
\begin{aligned}
N_{j}\left(K, \sigma_{x^{M}}, d_{p r}\right)= & \int_{-\infty}^{\infty} d x^{M} \operatorname{Eff}_{j}\left(x^{M}\right) \int_{0}^{\infty} d x G\left(x-x^{M}, \sigma_{x^{M}}\right) \\
& \cdot\left(p_{(d, u, s), j}^{o s c}\left(x, K, d_{p r}\right)+p_{(d, u, s), j}^{\text {nos }}\left(x, K, d_{p r}\right)\right)
\end{aligned}
$$


The total VPDL $p d f$ for the $\mu^{+} D_{s}^{-}$signal is a sum of all the contributions which give the $D_{s}$ mass peak:

$$
\begin{aligned}
P_{\mu D_{s}}^{o s c, n o s}\left(x^{M}, \sigma_{x^{M}}, d_{p r}\right)= & \left(\sum_{j} B r_{j} \cdot P_{d, j}^{o s c, n o s}\left(x^{M}, \sigma_{x^{M}}, d_{p r}\right)\right. \\
& +\sum_{j} B r_{j} \cdot P_{u, j}^{o s c, n o s}\left(x^{M}, \sigma_{x^{M}}, d_{p r}\right) \\
& \left.+\sum_{j} B r_{j} \cdot P_{s, j}^{o s c, n o s}\left(x^{M}, \sigma_{x^{M}}, d_{p r}\right)\right) \\
& \cdot\left(1-F r_{\text {peak }}\right)+F r_{\text {peak }} \cdot P_{\text {peak }}^{o s c, \text { nos }}\left(x^{M}\right)
\end{aligned}
$$

Here the sum $\sum_{j}$ is taken over all decay channels $B \rightarrow \mu^{+} \nu D_{s}^{-} X$ and the $B r_{j}$ is the branching rate of a given channel $j$. In addition to the long-lived $B$ candidates, there is a contribution of the "peaking background" which consists of combinations of $D_{s}$ mesons and muons originating from different $c$ or $b$ quarks. The direct $c$ production gives the largest contribution to this background; therefore the function $P_{\text {peak }}^{o s c}{ }^{n o s}\left(x^{M}\right)$ was determined from $c \bar{c} \mathrm{MC}$ (see Fig. 3.25). We assume that this background is not sensitive to tagging. The same $p d f$ was used in Reference [51] for studies of $B_{d}$ mixing.

The branching rates $B r_{j}$ were taken from the Particle Data Group (PDG) [6] and are

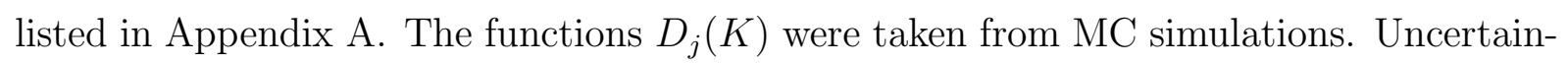
ties in all of these inputs will contribute to the systematic uncertainties and are discussed in Section 4.1

The $B$ meson lifetimes and efficiencies $E f f_{j}(x)$ are highly correlated. Previously, the efficiencies determined using MC did not take into account the trigger selection and therefore gave biased measurements of the $B$ meson lifetimes. We now use MC efficiencies with 
the trigger efficiency applied; details are given in the next section. Note that $\Delta m_{s}$ measurements are not directly influenced by the lifetime; the uncertainty on the $B_{s}^{0}$ lifetime in Ref. [59] is about $0.01 \mathrm{ps}$ which is much less than the uncertainty on $\Delta m_{s}$ measurements and so has minimal impact on the result. Therefore, we measure the $B_{s}^{0}$ lifetime in data using the MC efficiencies and include the deviation from the PDG value in the systematics.

\subsection{2. $p d f$ for Combinatorial Background}

The following contributions to the combinatorial background were considered:

(1) Prompt background with the $\mu^{+} D_{s}^{-}$vertex coinciding with the primary vertex (described as a Gaussian with a width determined by the resolution; fraction in the background: $\mathcal{F}_{0}$ ). The resolution scale factor for this background is different from the signal resolution scale factor. The scale factor is a free fit parameter, $s_{b k g}$. This background is caused by a fake secondary vertex, or a secondary vertex of unknown origin, combined with a muon originating from the primary vertex.

(2) Background with quasi-vertices distributed around the primary vertex (described as a Gaussian with constant width $\sigma_{\text {peak_bkg }}$; fraction in the background: $\mathcal{F}_{\text {peak_bkg }}$ ). This background is produced from direct $c \bar{c}$ production where one of the $c$ quarks decays to a muon and the other produces three or more displaced tracks.

(3) Long-lived background (exponential with constant decay length $c \tau_{b k g}$ convoluted with the resolution). This background was divided into three subsamples:

(a) insensitive to the tagging (fraction in the long-lived background: $\left(1-\mathcal{F}_{\text {tsens }}\right)$ ); 
(b) sensitive to the tagging and non-oscillating (fraction in the background sensitive to the tagging: $\left.\left(1-\mathcal{F}_{\text {osc }}\right)\right)$;

(c) sensitive to the tagging and oscillating with frequency $\Delta m_{d}$ (fraction in the background sensitive to the tagging: $\left.\mathcal{F}_{\text {osc }}\right)$.

The long-lived backgrounds come from either indirect $c \bar{c}$ production where one of the $c$ quarks decays to a muon (insensitive to the flavor tagging), or $B$ decays that are not $\mu^{+} D_{s}^{-}$(sensitive to the flavor tagging).

The fractions of these contributions and their parameters were determined from the data sample. The background $p d f$ was expressed in the following form:

$$
\begin{aligned}
& P_{b k g}\left(x^{M}, \sigma_{x^{M}}, \mathcal{D}\right)=\left(\mathcal{F}_{\text {peak_bkg }} G\left(x^{M}, \sigma_{\text {peak_bkg }}\right)\right. \\
& \left.+\left(1-\mathcal{F}_{\text {peak_bkg }}\right) \cdot P_{b k g}^{r e s}\left(x^{M}, \sigma_{x^{M}}\right)\right), \\
& P_{b k g}^{r e s}\left(x^{M}, \sigma_{x^{M}}, \mathcal{D}\right)=\frac{E f f\left(x^{M}\right)}{N} \int_{0}^{\infty} d x\left(\mathcal{F}_{0} G\left(x-x^{M}, s_{b k g} \sigma_{x^{M}}\right) \delta(x)\right. \\
& \left.+\left(1-\mathcal{F}_{0}\right) G\left(x-x^{M}, \sigma_{x^{M}}\right) \cdot p_{b k g}^{l o n g}\right), \\
& p_{\text {bkg }}^{\text {long,osc } / \text { nos }}(x, \mathcal{D})=e^{\left(-\frac{x}{c \tau_{b k g}}\right)}\left(\left(1-\mathcal{F}_{\text {tsens }}\right)+\mathcal{F}_{\text {tsens }}\left((1 \pm \mathcal{D})\left(1-\mathcal{F}_{\text {osc }}\right)\right.\right. \\
& \left.\left.+\left(1 \pm \mathcal{D} \cos \left(\Delta m_{d} \cdot x / c\right)\right) \cdot \mathcal{F}_{o s c}\right)\right)
\end{aligned}
$$

where fit parameters were $\mathcal{F}_{\text {peak_bkg }}, \sigma_{\text {peak_bkg }}, \mathcal{F}_{0}, \mathcal{F}_{\text {tsens }}, \mathcal{F}_{\text {osc }}$ and $c \tau_{\text {bkg }}$. As an efficiency Ef $f\left(x^{M}\right)$, the efficiency for the $B_{d} \rightarrow D^{ \pm} \mu \nu$ channel was used. 


\subsection{Inputs to the Fit}

\subsubsection{Muon Trigger Efficiency Curves}

The data sample used for this analysis has no specific trigger requirements. As mentioned in Section 2.2.7 different triggers have different prescales and efficiencies at different $p_{T}$. Studies were done [60, 61, using both RunIIa and RunIIb data, to account for these behaviors across $p_{T}$. Distributions of triggered events were divided by unbiased events to create the trigger efficiency curve. These curves were then fitted to obtain the reweighting functions for RunIIa and RunIIb data.

The reweighting curves are shown in Figure 3.26, with RunIIa on the left and RunIIb on the right. There are a large number of curves, each one depending on a different set of background reduction cuts. The very central curve of each distribution is used as the default curve, and is applied to the sample composition and $K$ factor distributions. The trigger efficiencies are different in RunIIa and RunIIb, as can be seen in Figure 3.26. The RunIIb efficiency has a sharper turn-on and plateaus at lower $p_{T}$ than the RunIIa curve. Note that the variation in the efficiency curve for RunIIa and RunIIb is taken into account in the systematics, described in Section 4.1.

\subsection{2. $K$ Factor}

There is an undetected neutrino in all semileptonic $B$ decays, which makes a precise determination of the $B$ momentum impossible. There can also be other undetected neutral or unreconstructed charged decay particles. These missing particles cause a bias in the $B$ momentum, which is calculated from reconstructed particles, towards smaller values. To correct for the missing momentum, the $B$ momentum is scaled by a scaling factor called 

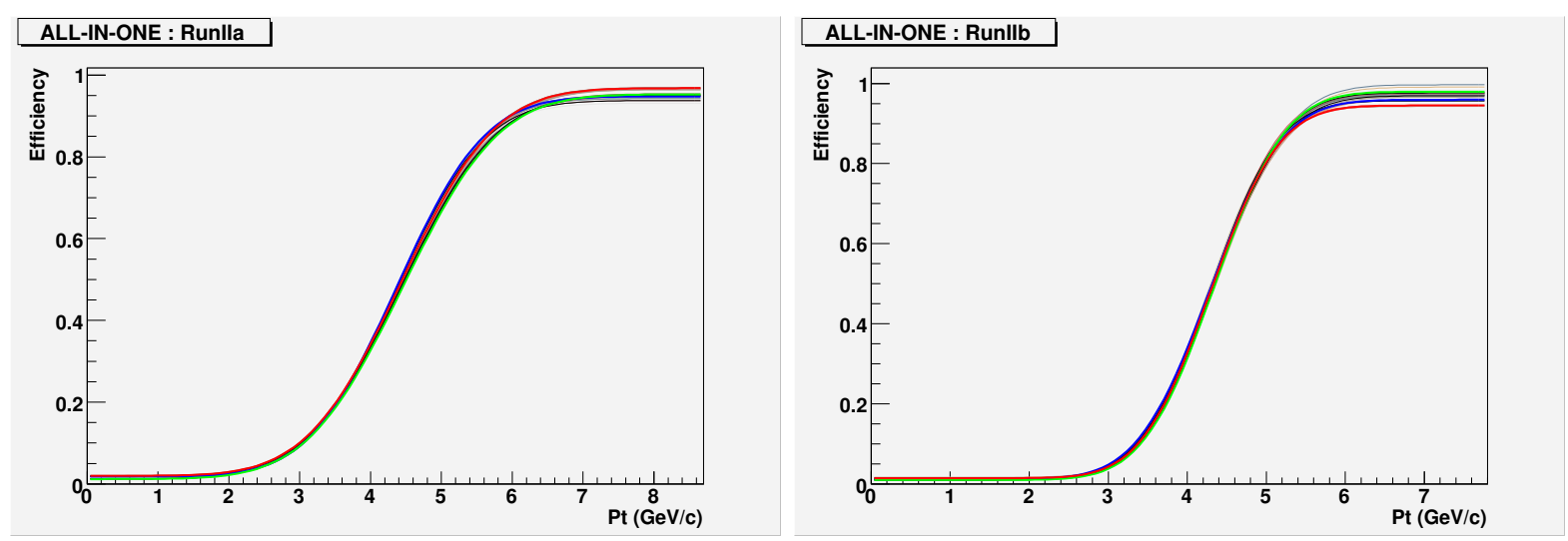

Figure 3.26. The trigger turn-on curves. RunIIa is on the left and RunIIb on the right. Each color represents a different selection cut combination; the central curve is used as the default.

a $K$ factor. The $K$ factor is estimated using a MC sample, and is defined as:

$$
K=p_{T}\left(\mu^{+} D_{s}^{-}\right) / p_{T}\left(B_{s}^{0}\right)
$$

where $p_{T}$ is the transverse momentum. Because the $K$ factors are defined as the ratio of $p_{T} \mathrm{~s}$, they can exceed unity.

A number of improvements to the $K$ factors have been made since the previous $B_{s}^{0}$ mixing result in Reference [17, and are explained in detail in Reference [62]. Previously, the reconstructed value of $p_{T}$ was used; now, the generator-level information is used. This introduces only a minimal bias and has the advantage of allowing large-statistics MC samples to be produced much more quickly. The higher statistics allow finer binning in both $m\left(D_{s}^{-}\right)$and $K$ factor, which in turn allows the elimination of the (previously necessary) systematic to account for the large bins used in previous $B_{s}^{0}$ mixing studies. 
Following Equation 3.33, the $K$ factors are constructed for each of the ten channels described in Section 3.6.3. after the lifetime cuts have been applied3. Additionally, the muon trigger efficiency curves [60, 61] were applied.

$K$ factor distributions for $B_{s}^{0} \rightarrow D_{s}^{-} \mu^{+} \nu$ and $B_{s}^{0} \rightarrow D_{s}^{*-} \mu^{+} \nu \rightarrow D_{s}^{-} \mu^{+} \nu$ decays are shown in Figure 3.27. The $D_{s}^{*-} K$ factors have a lower mean value than the $D_{s}^{-} K$ factors since fewer decay products are reconstructed.

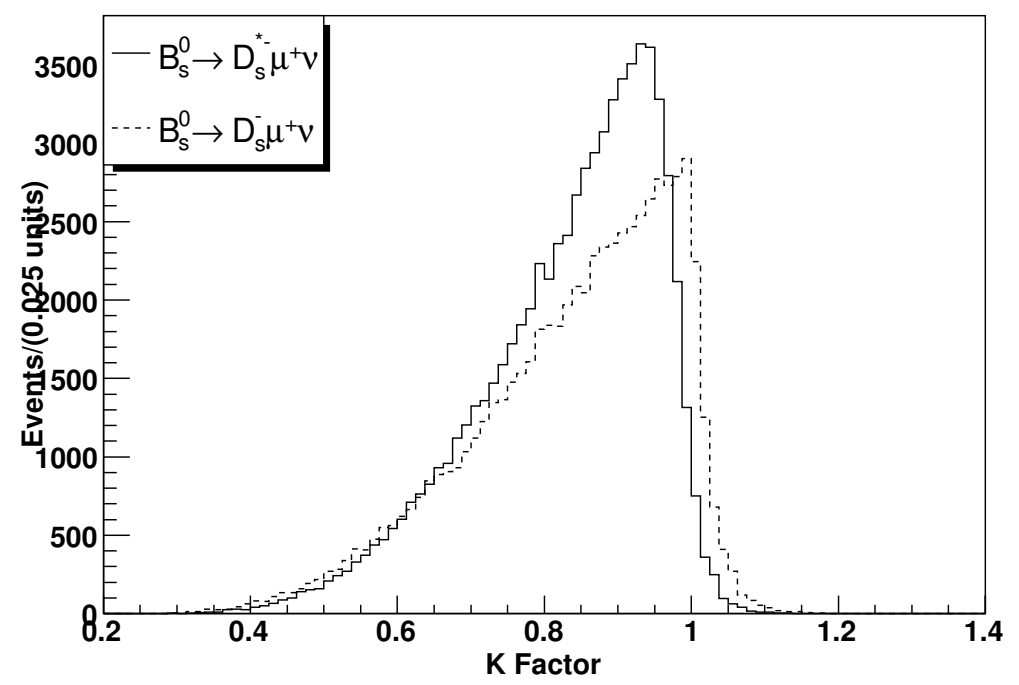

Figure 3.27. $K$ factors for $B_{s}^{0} \rightarrow \mu^{+} \nu D_{s}^{-}($mean $=0.856, R M S=0.153)$ and $B_{s}^{0} \rightarrow \mu^{+} \nu D_{s}^{*-} \rightarrow \mu^{+} \nu D_{s}^{-}($mean $=0.825, R M S=0.138)$ processes after the application of the p17 muon trigger turn-on curve.

The $K$ factors are dependent upon the mass $m\left(\mu^{+} D_{s}^{-}\right)$. This dependence, for signal $B_{s}^{0} \rightarrow D_{s}^{-} \mu \nu$ decays, is shown in Figure 3.28. The same dependence for $B_{s}^{0} \rightarrow \mu^{+} \nu D_{s}^{*-} \rightarrow$ $\mu^{+} \nu D_{s}^{-}$decays is shown in Figure 3.29 The figures show that the $K$ factor distributions are narrower for larger $m\left(\mu^{+} D_{s}^{-}\right)$values, since more of the momentum is carried by the reconstructed $\mu^{+} D_{s}^{-}$system. This variation in $K$ factor width is exploited in the $K$ factor ${ }^{3}$ Studies by Sergey Burdin and Wendy Taylor have shown that the lifetime cuts do not bias the $K$ factors. 


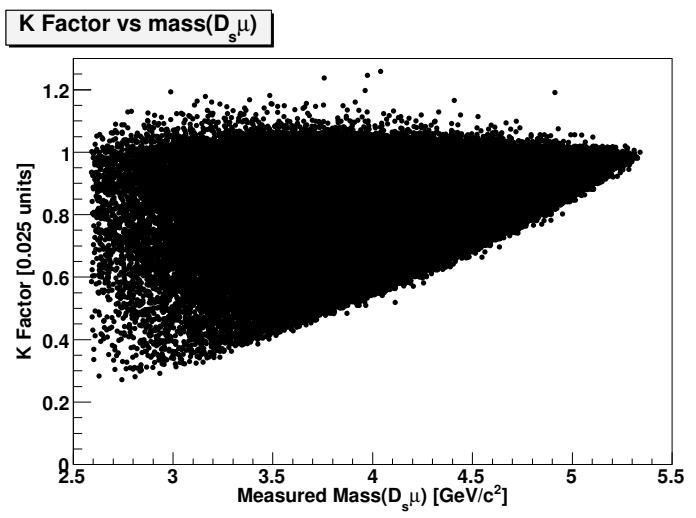

Figure 3.28. $K$ factor versus $\operatorname{mass}\left(\mu^{+} D_{s}^{-}\right)$ for $B_{s}^{0} \rightarrow \mu^{+} D_{s}^{-} \nu$ decays after the application of the p17 muon trigger turn-on curve.
K Factor vs mass $\left(D_{s} \mu\right)$

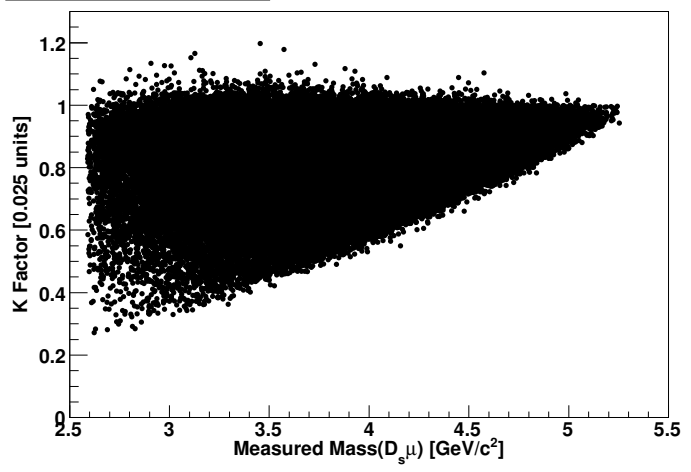

Figure 3.29. $K$ factor versus $\operatorname{mass}\left(\mu^{+} D_{s}^{-}\right)$for $B_{s}^{0} \rightarrow$ $\mu^{+} \nu D_{s}^{*-} \rightarrow \mu^{+} \nu D_{s}^{-}$decays after the application of the p17 muon trigger turn-on curve.

construction. The $K$ factors used in this analysis are divided into ten bins of $\mu^{+} D_{s}^{-}$mass, whereas the previous analysis used only four mass bins. The range of the ten mass bins is given in Table 3.2, and an example of the $K$ factors in each of the ten mass bins for RunIIa data is given in Figure 3.30 for the decay $B_{s}^{0} \rightarrow \mu^{+} D_{s}^{-} \nu$ and in Figure 3.31 for the decay $B_{s}^{0} \rightarrow \mu^{+} \nu D_{s}^{*-} \rightarrow \mu^{+} \nu D_{s}^{-}$.

Table 3.2. $\operatorname{Mass}\left(\mu^{+} D_{s}^{-}\right)$Bins

\begin{tabular}{|c|c|}
\hline bin 1 & $\operatorname{mass}\left(D_{s}^{-} \mu^{+}\right) \leq 2.95 \mathrm{GeV} / c^{2}$ \\
bin 2 & $2.95<\operatorname{mass}\left(D_{s}^{-} \mu^{+}\right) \leq 3.20 \mathrm{GeV} / c^{2}$ \\
bin 3 & $3.20<\operatorname{mass}\left(D_{s}^{-} \mu^{+}\right) \leq 3.45 \mathrm{GeV} / c^{2}$ \\
bin 4 & $3.45<\operatorname{mass}\left(D_{s}^{-} \mu^{+}\right) \leq 3.70 \mathrm{GeV} / c^{2}$ \\
bin 5 & $3.70<\operatorname{mass}\left(D_{s}^{-} \mu^{+}\right) \leq 3.95 \mathrm{GeV} / c^{2}$ \\
bin 6 & $3.95<\operatorname{mass}\left(D_{s}^{-} \mu^{+}\right) \leq 4.20 \mathrm{GeV} / c^{2}$ \\
bin 7 & $4.20<\operatorname{mass}\left(D_{s}^{-} \mu^{+}\right) \leq 4.45 \mathrm{GeV} / c^{2}$ \\
bin 8 & $4.45<\operatorname{mass}\left(D_{s}^{-} \mu^{+}\right) \leq 4.70 \mathrm{GeV} / c^{2}$ \\
bin 9 & $4.70<\operatorname{mass}\left(D_{s}^{-} \mu^{+}\right) \leq 4.95 \mathrm{GeV} / c^{2}$ \\
bin 10 & $\operatorname{mass}\left(D_{s}^{-} \mu^{+}\right)>4.95 \mathrm{GeV} / c^{2}$ \\
\hline
\end{tabular}



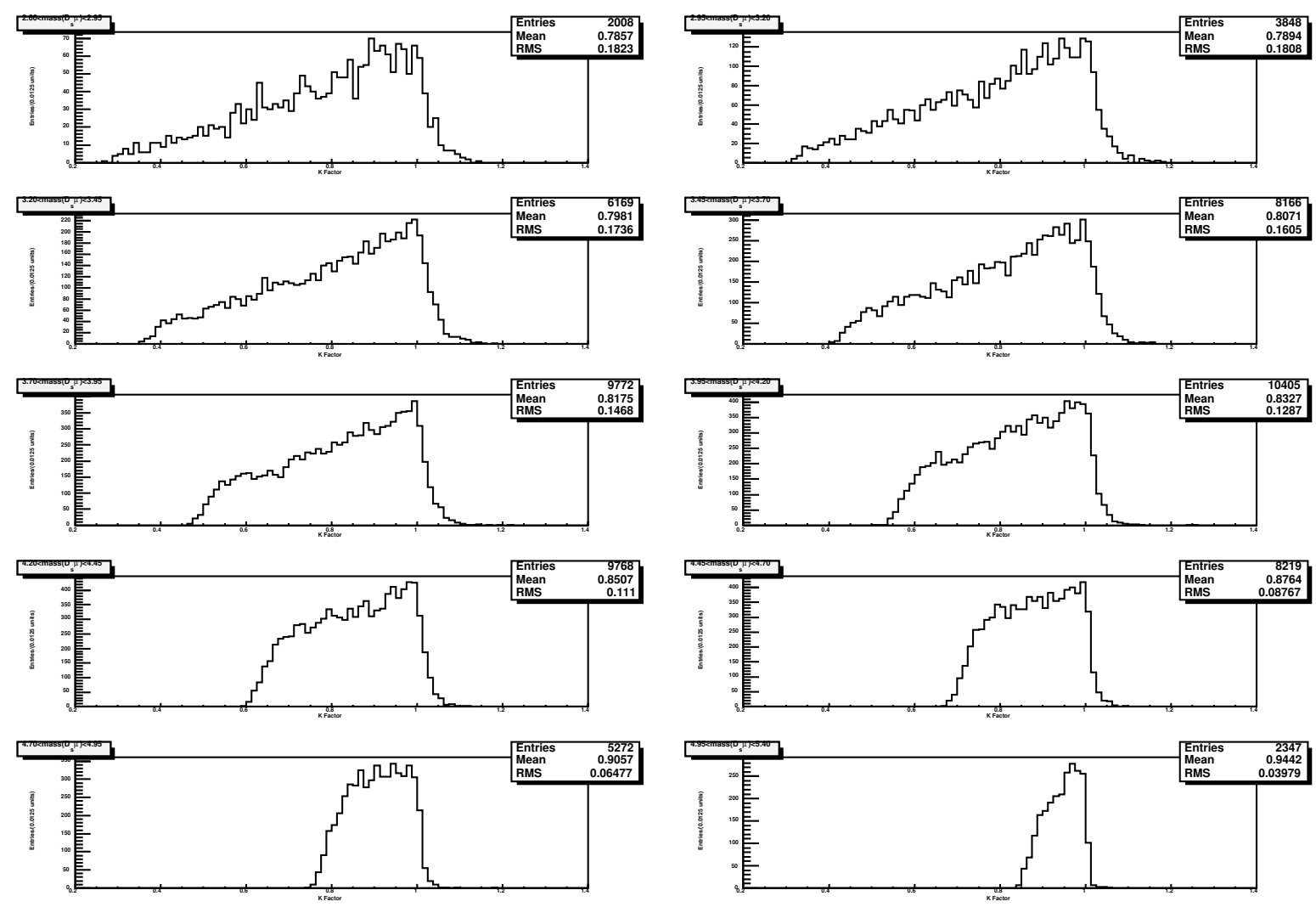

Figure 3.30. $K$ factor versus $m\left(D_{s}^{-} \mu^{+}\right)$for $B_{s}^{0} \rightarrow \mu^{+} \nu D_{s}^{-}$decays after the application of the p17 muon trigger turn-on curve. The plots show the $K$ factor distribution in each of the ten $\mu^{+} D_{s}^{-}$mass bins, from the lowest-mass bin in the upper-left corner to the highest-mass bin in the lower-right corner.

The $K$ factor distributions for each of the ten channels listed in Section 3.6 .3 were used as inputs to the fit. The distributions used were in a two-dimentional histogram form for each of the probability equations given in Equations 3.13 - 3.23, in ten bins of $m\left(D_{s}^{-} \mu\right)$ and ninety-six $K$ factor bins. Since RunIIa and RunIIb data utilize different muon trigger efficiency curves and MC versions, separate $K$ factor distributions were constructed for the two data collection periods. 

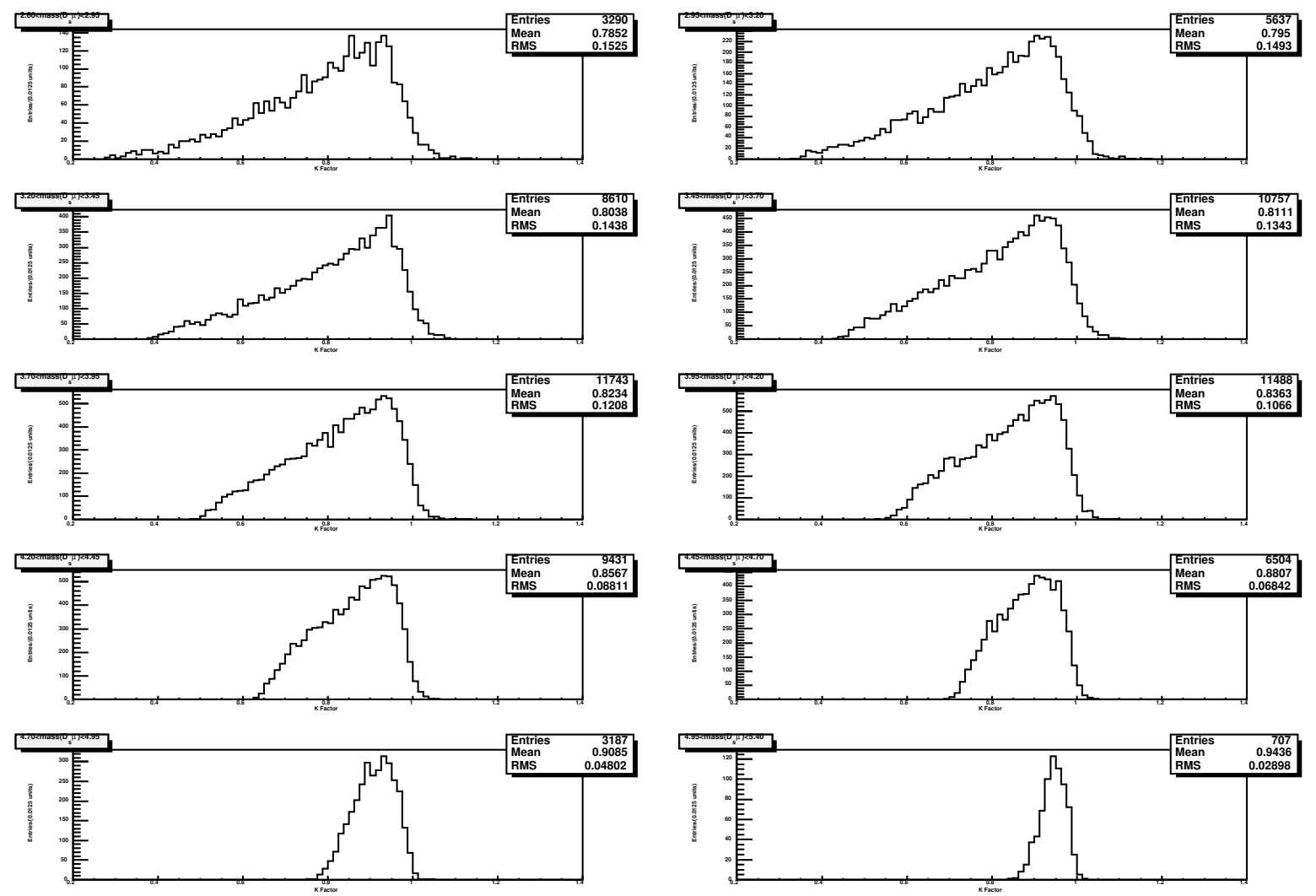

Figure 3.31. $K$ factor versus $m\left(D_{s}^{-} \mu^{+}\right)$for $B_{s}^{0} \rightarrow \mu^{+} \nu D_{s}^{*-} \rightarrow \mu^{+} \nu D_{s}^{-}$decays after the application of the p17 muon trigger turn-on curve. The plots show the $K$ factor distribution in each of the ten $\mu^{+} D_{s}^{-}$mass bins, from the lowest-mass bin in the upper-left corner to the highest-mass bin in the lower-right corner.

\subsubsection{Sample Composition}

The following ten $B$ meson decay channels were considered in the determination of the sample composition of the $D_{s}^{-} \mu$ sample:

- $B_{s}^{0} \rightarrow \mu^{+} \nu D_{s}^{-}$;

- $B_{s}^{0} \rightarrow \mu^{+} \nu D_{s}^{-*} \rightarrow \mu^{+} \nu D_{s}^{-}$;

- $B_{s}^{0} \rightarrow \mu^{+} \nu D_{s 0}^{*-} \rightarrow \mu^{+} \nu D_{s}^{-}$; 
- $B_{s}^{0} \rightarrow \mu^{+} \nu D_{s 1}^{\prime} \rightarrow \mu^{+} \nu D_{s}^{-}$

- $B_{s}^{0} \rightarrow \tau^{+} \nu D_{s}^{-} X, \tau \rightarrow \mu \nu \nu$;

- $B_{s}^{0} \rightarrow D_{s}^{+} D_{s}^{-} X ; D_{s}^{-} \rightarrow \mu \nu X$;

- $B_{s}^{0} \rightarrow D_{s}^{-} D X ; D \rightarrow \mu^{+} \nu X$;

- $\bar{B}_{s}^{0} \rightarrow D_{s}^{-} D X ; D \rightarrow \mu^{+} \nu X$;

- $B^{+} \rightarrow D D_{s}^{-} X ; D \rightarrow \mu \nu X$;

- $B^{0} \rightarrow D D_{s}^{-} X ; D \rightarrow \mu \nu X$.

To determine the branching fractions of contributing decays, the most recent PDG values [6] were used when available. For branching fractions not listed in the PDG, EvtGen [50] inputs were used. The branching fractions and their origins are listed in Appendix $\mathrm{A}$

To estimate the sample composition uncertainty, each branching fraction used was varied by its uncertainty. The maximum deviation that resulted in the sample composition was used as the sample composition uncertainty. Note that we did not take into account the $\mathrm{MC}$ statistical uncertainty. For the $D D$ branching fractions that were estimated from EvtGen, a 25\% relative uncertainty was used 63. Variations in the signal $B_{s}^{0} \rightarrow D_{s}^{-} \mu^{+} \nu X$ branching fractions were not computed, as the variation of the respective $K$ factors already takes this into account. It should be noted that for most contributions, varying the signal branching fraction within its uncertainty of $(7.9 \pm 2.4) \%$ causes the maximum deviation in the sample composition; this is taken into account in the systematics.

In previous $B_{s}^{0}$ mixing studies, the sample composition systematics included a study of the sensitivity to the muon $p_{T}$. However, since the muon trigger turn-on curves are

\footnotetext{
${ }^{4}$ Note that the $D_{s 1}^{\prime-}$ state corresponds to the $D_{s 1}^{-}(2460)$ state in the PDG.
} 


\begin{tabular}{|c|c|c|c|c|c|c|c|c|c|c|}
\hline \multicolumn{10}{|c|}{ p17 Sample Composition for the 10 mass $\left(D_{s} \mu\right)$ bins } \\
\hline \hline & bin 1 & bin 2 & bin 3 & bin 4 & bin 5 & bin 6 & bin 7 & bin 8 & bin 9 & bin 10 \\
\hline$B_{s}^{0} \rightarrow D_{s}^{+} \mu X$ & $10.5 \%$ & $14.8 \%$ & $16.8 \%$ & $18.5 \%$ & $20.4 \%$ & $22.5 \%$ & $25.6 \%$ & $30.3 \%$ & $37 . \%$ & $54.6 \%$ \\
\hline$B_{s}^{0} \rightarrow D_{s}^{*+} \mu X$ & $47.3 \%$ & $59.6 \%$ & $64.6 \%$ & $66.9 \%$ & $67.6 \%$ & $68.4 \%$ & $68 . \%$ & $66 . \%$ & $61.5 \%$ & $45.2 \%$ \\
\hline$B_{s}^{0} \rightarrow D_{s 0}^{*+} \mu X$ & $1.2 \%$ & $1.6 \%$ & $1.7 \%$ & $1.8 \%$ & $1.8 \%$ & $1.7 \%$ & $1.4 \%$ & $1.0 \%$ & $0.6 \%$ & $0.1 \%$ \\
\hline$B_{s}^{0} \rightarrow D_{s 1}^{+} \mu X$ & $2.4 \%$ & $2.7 \%$ & $3.2 \%$ & $3.9 \%$ & $4.5 \%$ & $4.5 \%$ & $3.8 \%$ & $2.2 \%$ & $0.8 \%$ & $0.1 \%$ \\
\hline$B_{s}^{0} \rightarrow D_{s}^{+} \tau X$ & $3.3 \%$ & $2.0 \%$ & $1.3 \%$ & $0.7 \%$ & $0.4 \%$ & $0.2 \%$ & $0.1 \%$ & $0.0 \%$ & $0.0 \%$ & $0.0 \%$ \\
\hline$B_{s}^{0} \rightarrow D_{s}^{+} D_{s}^{-} X$ & $1.2 \%$ & $1.1 \%$ & $1.0 \%$ & $0.8 \%$ & $0.6 \%$ & $0.4 \%$ & $0.3 \%$ & $0.3 \%$ & $0.2 \%$ & $0.0 \%$ \\
\hline$B_{s}^{0} \rightarrow D_{s}^{+} D^{-} X$ & $3.7 \%$ & $1.1 \%$ & $0.3 \%$ & $0.1 \%$ & $0.0 \%$ & $0.0 \%$ & $0.0 \%$ & $0.0 \%$ & $0.0 \%$ & $0.0 \%$ \\
\hline$B_{s}^{0} \rightarrow D_{s}^{+} D^{-} X$ & $3.6 \%$ & $1.3 \%$ & $0.5 \%$ & $0.2 \%$ & $0.1 \%$ & $0.1 \%$ & $0.0 \%$ & $0.0 \%$ & $0.0 \%$ & $0.0 \%$ \\
\hline$B^{+} \rightarrow D_{s}^{+} D^{-} X$ & $11.3 \%$ & $6.5 \%$ & $4.5 \%$ & $3.2 \%$ & $2.0 \%$ & $1.1 \%$ & $0.4 \%$ & $0.1 \%$ & $0.0 \%$ & $0.0 \%$ \\
\hline$B^{0} \rightarrow D_{s}^{+} D^{-} X$ & $15.6 \%$ & $9.2 \%$ & $6.0 \%$ & $4.0 \%$ & $2.5 \%$ & $1.3 \%$ & $0.5 \%$ & $0.1 \%$ & $0.0 \%$ & $0.0 \%$ \\
\hline
\end{tabular}

Table 3.3. Contributions of each decay chain to the total $B_{s}^{0}$ signal, for each $\operatorname{mass}\left(D_{s}^{-} \mu^{+}\right)$bin for $\mathrm{p} 17$

now included in the construction of the sample composition, this dependence is already accounted for.

Using the ten $m\left(D_{s}^{-} \mu\right)$ mass bins defined for the $K$ factors in Table 3.2 the fractional contribution of each decay to the total $B_{s}^{0}$ signal was determined using the equation:

$$
F_{b i n j}^{i}=\frac{f_{j}^{i} \cdot F^{i}}{\sum_{i}\left(f_{j}^{i} \cdot F^{i}\right)}
$$

where $F_{b i n j}^{i}$ is the fraction of the total events in the $j^{\text {th }}$ bin of the $i^{\text {th }}$ decay chain, $f_{j}^{i}$ is the fraction of events from the $i^{\text {th }}$ decay chain that fall into the $j^{\text {th }}$ bin, and $F^{i}$ is the overall (unbinned) contribution of the $i^{\text {th }}$ decay chain to the total signal. The sample composition for the ten bins of mass $\left(D_{s}^{-} \mu^{+}\right)$is given for RunIIa in Table 3.3 and for RunIIb in Table 3.4 . 


\begin{tabular}{|c|c|c|c|c|c|c|c|c|c|c|}
\hline \multicolumn{10}{|c|}{ p20 Sample Composition for the 10 mass $\left(D_{s} \mu\right)$ bins } \\
\hline \hline & bin 1 & bin 2 & bin 3 & bin 4 & bin 5 & bin 6 & bin 7 & bin 8 & bin 9 & bin 10 \\
\hline$B_{s}^{0} \rightarrow D_{s}^{+} \mu X$ & $8.6 \%$ & $13.2 \%$ & $15.3 \%$ & $17.2 \%$ & $19.5 \%$ & $21.5 \%$ & $24.6 \%$ & $29.5 \%$ & $36.3 \%$ & $53.6 \%$ \\
\hline$B_{s}^{0} \rightarrow D_{s}^{*+} \mu X$ & $39.8 \%$ & $54.9 \%$ & $61.6 \%$ & $64.8 \%$ & $66.5 \%$ & $68.2 \%$ & $68.5 \%$ & $66.7 \%$ & $62.1 \%$ & $46.1 \%$ \\
\hline$B_{s}^{0} \rightarrow D_{s 0}^{*+} \mu X$ & $1.1 \%$ & $1.6 \%$ & $1.8 \%$ & $1.9 \%$ & $1.9 \%$ & $1.8 \%$ & $1.5 \%$ & $1.1 \%$ & $1.0 \%$ & $0.2 \%$ \\
\hline$B_{s}^{0} \rightarrow D_{s 1}^{+} \mu X$ & $1.9 \%$ & $2.3 \%$ & $2.8 \%$ & $3.6 \%$ & $4.2 \%$ & $4.3 \%$ & $3.6 \%$ & $2.1 \%$ & $0.7 \%$ & $0.1 \%$ \\
\hline$B_{s}^{0} \rightarrow D_{s}^{+} \tau X$ & $4.6 \%$ & $3.0 \%$ & $2.0 \%$ & $1.2 \%$ & $0.6 \%$ & $0.3 \%$ & $0.1 \%$ & $0.0 \%$ & $0.0 \%$ & $0.0 \%$ \\
\hline$B_{s}^{0} \rightarrow D_{s}^{+} D_{s}^{-} X$ & $1.2 \%$ & $1.2 \%$ & $1.0 \%$ & $0.9 \%$ & $0.7 \%$ & $0.5 \%$ & $0.4 \%$ & $0.3 \%$ & $0.2 \%$ & $0.0 \%$ \\
\hline$B_{s}^{0} \rightarrow D_{s}^{+} D^{-} X$ & $5.4 \%$ & $1.8 \%$ & $0.5 \%$ & $0.1 \%$ & $0.0 \%$ & $0.0 \%$ & $0.0 \%$ & $0.0 \%$ & $0.0 \%$ & $0.0 \%$ \\
\hline$B_{s}^{0} \rightarrow D_{s}^{+} D^{-} X$ & $5.2 \%$ & $2.0 \%$ & $0.8 \%$ & $0.4 \%$ & $0.2 \%$ & $0.1 \%$ & $0.1 \%$ & $0.0 \%$ & $0.0 \%$ & $0.0 \%$ \\
\hline$B^{+} \rightarrow D_{s}^{+} D^{-} X$ & $13.6 \%$ & $8.4 \%$ & $6.1 \%$ & $4.4 \%$ & $2.8 \%$ & $1.5 \%$ & $0.5 \%$ & $0.1 \%$ & $0.0 \%$ & $0.0 \%$ \\
\hline$B^{0} \rightarrow D_{s}^{+} D^{-} X$ & $18.6 \%$ & $11.7 \%$ & $8.1 \%$ & $5.6 \%$ & $3.5 \%$ & $1.8 \%$ & $0.7 \%$ & $0.2 \%$ & $0.0 \%$ & $0.0 \%$ \\
\hline
\end{tabular}

Table 3.4. Contributions of each decay chain to the total $B_{s}^{0}$ signal, for each $\operatorname{mass}\left(D_{s}^{-} \mu^{+}\right)$bin for $\mathrm{p} 20$

\subsubsection{Reconstruction Efficiencies}

MC samples were used to determine the reconstruction efficiency of each contribution in the $B_{s}^{0}$ sample, for both RunIIa and RunIIb data. The reconstruction efficiency is very dependent upon the decay length; this is because of the lifetime-biased selections of the sample5. The reconstruction efficiencies are calculated as a function of the reconstructed VPDL and fitted with the function:

$$
\operatorname{Eff}\left(x^{M}\right)=p_{0} \cdot\left(1-\left(p_{2}+p_{3} \cdot x^{M}+p_{4} \cdot\left(x^{M}\right)^{2}+p_{5} \cdot\left(x^{M}\right)^{3}\right) \cdot \exp \left(-\left(x^{M}\right)^{2} / p_{1}\right)\right) .
$$

This choice of fitting function allows an analytical determination of the normalization integral in Equation 3.31.

Figures 3.32 and 3.33 show the efficiency as function of VPDL for the decay $B_{s} \rightarrow$ $\mu^{+} \nu D_{s}^{-} X$, for RunIIa and RunIIb respectively. All semileptonic modes were considered together.

\footnotetext{
${ }^{5}$ These are the IP significance cuts and $D_{s}^{-}$decay length cut in Section 3.2 additionally, the inclusion of events biased by impact parameter biasing triggers is discussed in Section 4.2 .2
} 


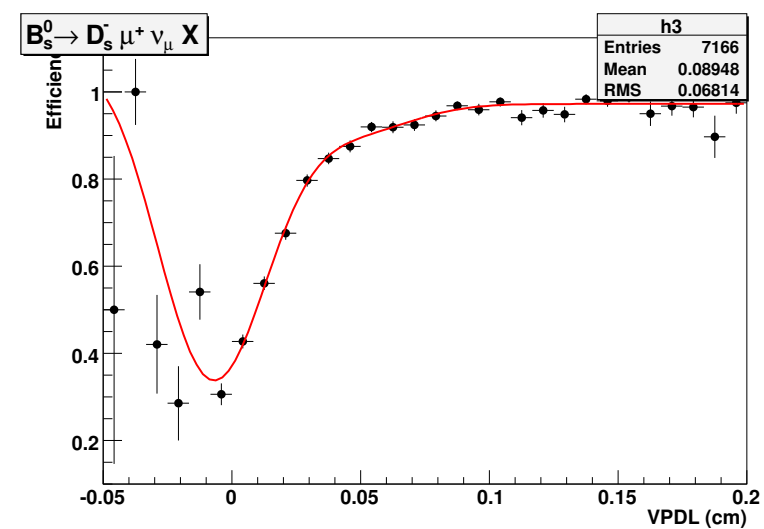

Figure 3.32. Efficiency as a function of VPDL for $B_{s} \rightarrow$ $\mu^{+} \nu D_{s}^{-} X$ in RunIIa.

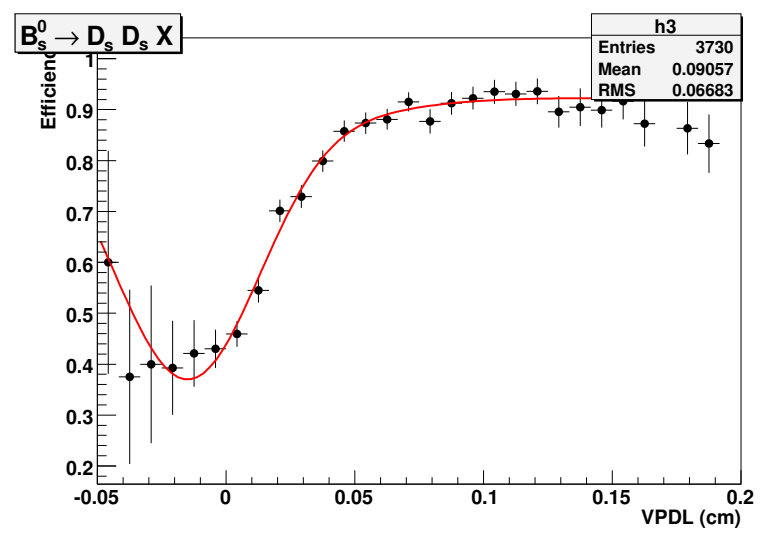

Figure 3.34. Efficiency as a function of VPDL for $B_{s} \rightarrow$ $D_{s}^{+} D_{s}^{-} X$ in RunIIa.

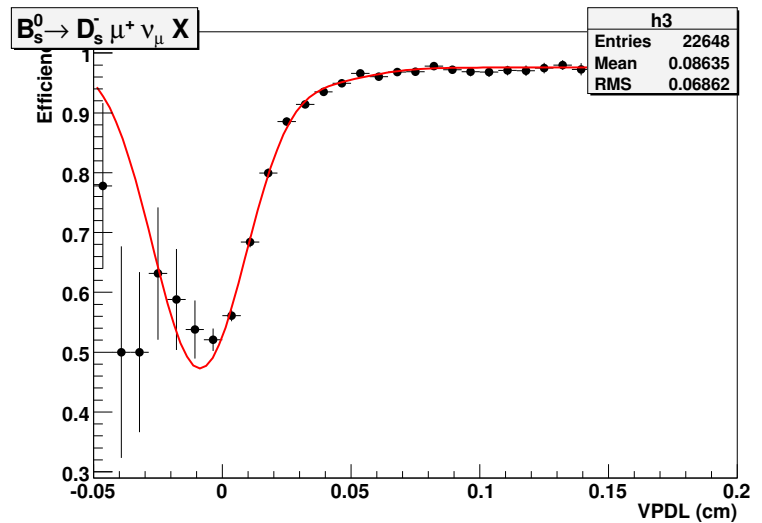

Figure 3.33. Efficiency as a function of VPDL for $B_{s} \rightarrow$ $\mu^{+} \nu D_{s}^{-} X$ in RunIIb.

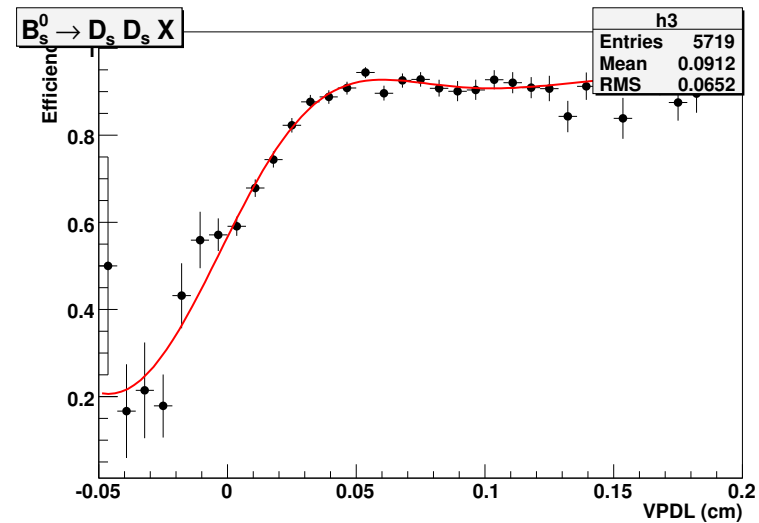

Figure 3.35. Efficiency as a function of VPDL for $B_{s} \rightarrow$ $D_{s}^{+} D_{s}^{-} X$ in RunIIb.

Figures 3.34 and 3.35 show the efficiency as function of VPDL for the decay $B_{s} \rightarrow$ $D_{s}^{+} D_{s}^{-} X, D_{s}^{-} \rightarrow \mu \nu X$ for RunIIa and RunIIb data respectively.

Figures 3.36 and 3.37 show the efficiency as function of VPDL for the decay $B_{s} \rightarrow$ $D D_{s}^{-} X ; D \rightarrow \mu^{+} \nu X$ for RunIIa and RunIIb data respectively. Additionally, the $\bar{B}_{s}^{0} \rightarrow$ $D D_{s}^{-} X, D \rightarrow \mu^{+} \nu X$ decay contributes, and its reconstruction efficiency for RunIIb is 
shown in Figure 3.38 For RunIIa, it was decided the efficiency in Figure 3.36 would be used for both the $B_{s}^{0}$ and $\bar{B}_{s}^{0}$ decays.

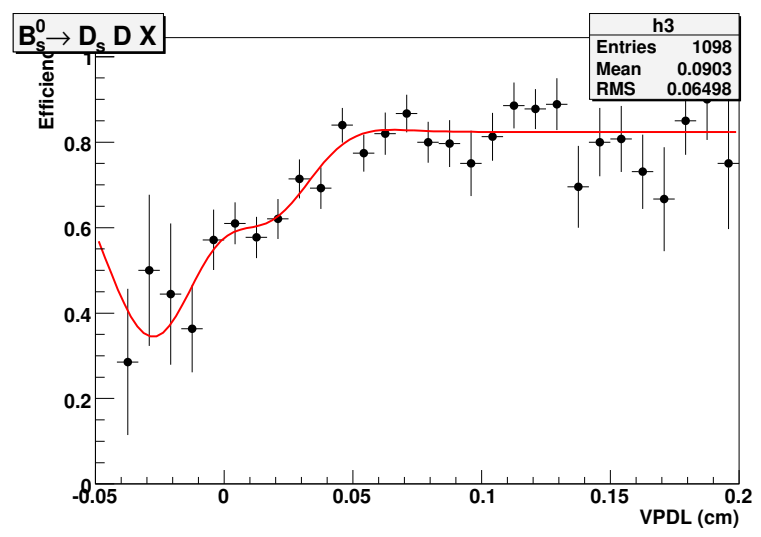

Figure 3.36. Efficiency as a function of VPDL for $B_{s} \rightarrow$ $D D_{s}^{-} X ; D \rightarrow \mu \nu X$ for RunIIa.

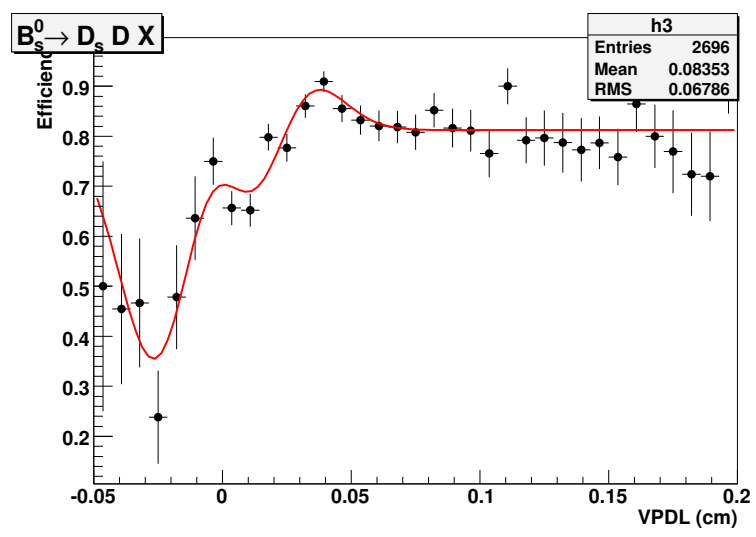

Figure 3.37. Efficiency as a function of VPDL for $B_{s} \rightarrow$ $D D_{s}^{-} X ; D \rightarrow \mu \nu X$ for RunIIb.

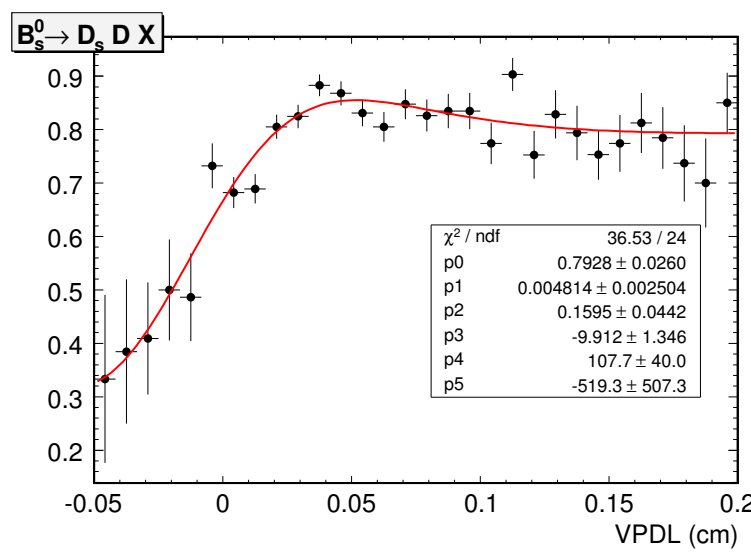

Figure 3.38. Efficiency as a function of VPDL for $\bar{B}_{s}^{0} \rightarrow$ $D D_{s}^{-} X ; D \rightarrow \mu \nu X$ for RunIIb. 


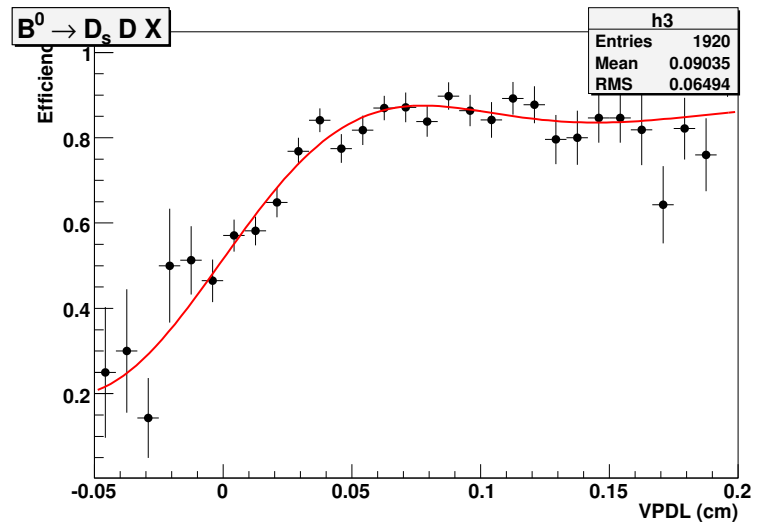

Figure 3.39. Efficiency as a function of VPDL for $B^{0} \rightarrow$ $D D_{s}^{-} X ; D \rightarrow \mu \nu X$ for RunIIa.

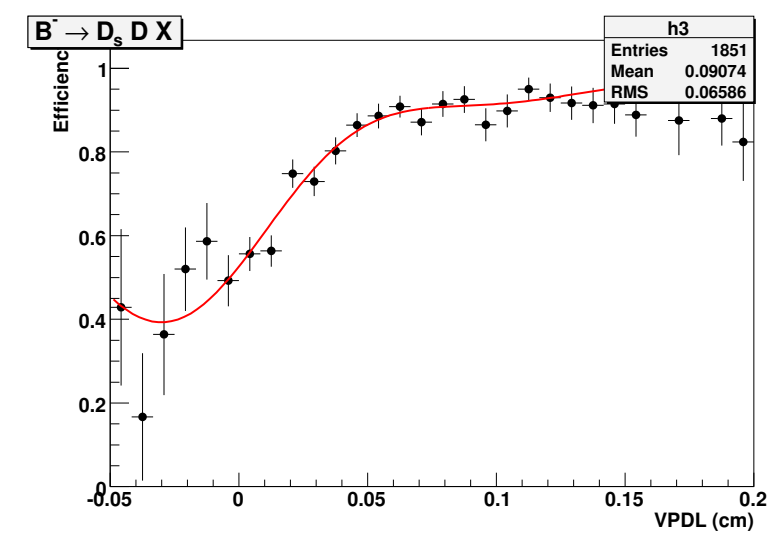

Figure 3.41. Efficiency as a function of VPDL for $B^{-} \rightarrow$ $D D_{s}^{-} X ; D \rightarrow \mu \nu X$ for RunIIa.

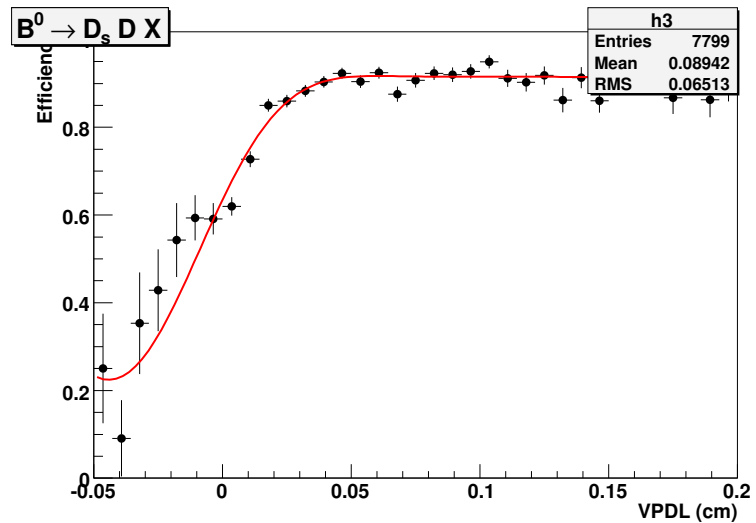

Figure 3.40. Efficiency as a function of VPDL for $B^{0} \rightarrow$ $D D_{s}^{-} X ; D \rightarrow \mu \nu X$ for RunIIb.

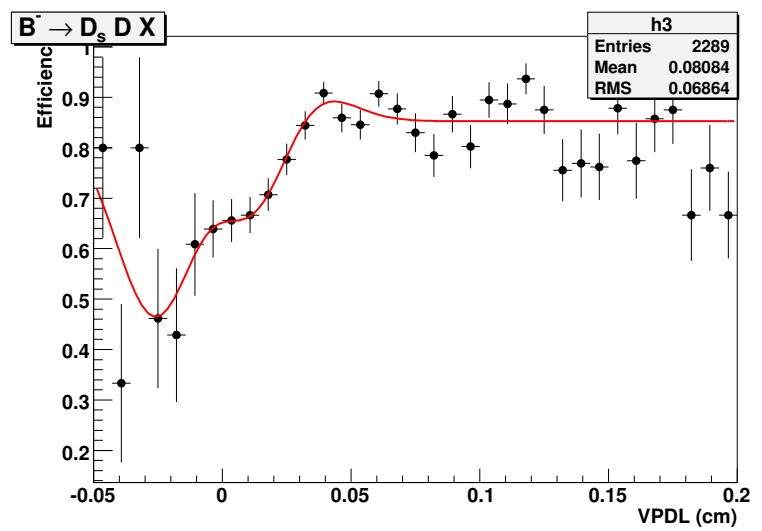

Figure 3.42. Efficiency as a function of VPDL for $B^{-} \rightarrow$ $D D_{s}^{-} X ; D \rightarrow \mu \nu X$ for RunIIb.

Figures 3.39 and 3.40 show the efficiency as function of VPDL for the decay $B^{0} \rightarrow$ $D D_{s}^{-} X ; D \rightarrow \mu \nu X$ for RunIIa and RunIIb respectively. The RunIIa and RunIIb reconstruction efficiencies for the charged decay $B^{-} \rightarrow D D_{s}^{-} X ; D \rightarrow \mu \nu X$ are shown in Figures 3.41 and 3.42 respectively. Finally, Figures 3.43 and 3.44 show the efficiency as function of VPDL for the decay $B_{s}^{0} \rightarrow \tau D_{s}^{-} X$, where $\tau \rightarrow \mu$, for RunIIa and RunIIb 


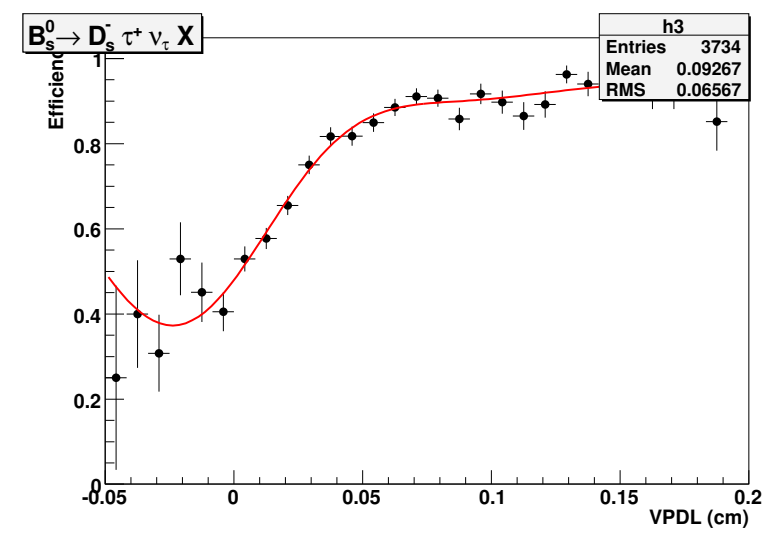

Figure 3.43. Efficiency as a function of VPDL for $B_{s}^{0} \rightarrow$ $\tau D_{s}^{-} X$, where $\tau \rightarrow \mu$ for RunIIb.

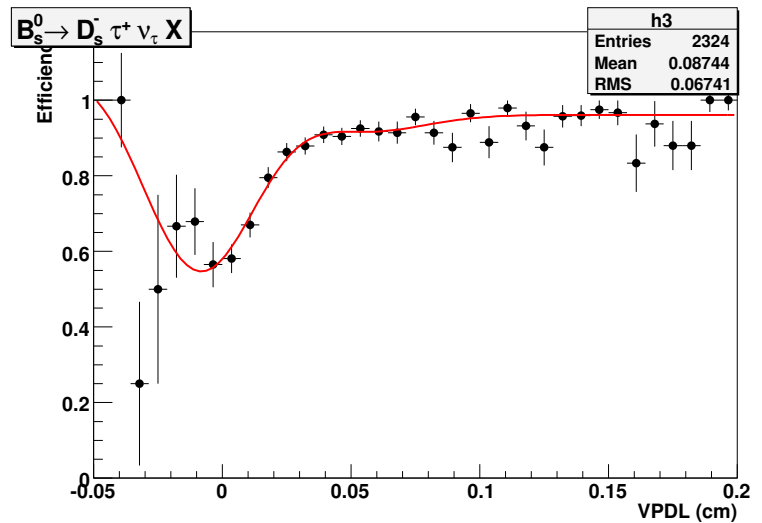

Figure 3.44. Efficiency as a function of VPDL for $B_{s}^{0} \rightarrow$ $\tau D_{s}^{-} X$, where $\tau \rightarrow \mu$ for RunIIb.

respectively. The tau decay efficiencies were not available in the previous analysis; these efficiencies represent an additional improvement to the analysis.

From all of these figures, the improvement in RunIIb reconstruction efficiency can easily be seen. The reconstruction efficiency in RunIIb at zero VPDL is greater than in the RunIIa distributions, and is due to the additional layer of silicon, Layer $\varnothing$, installed at the beginning of the collection of the RunIIb data.

\subsubsection{Resolution Scale Factor}

For the RunIIa data, an event-by-event scale factor $(S F)$ was used to scale the estimated impact parameter uncertainty from the tracking algorithm to the actual impact parameter uncertainty, as the tracking usually underestimates this uncertainty. Detailed information on the method used to obtain the event-by-event scale factor can be found in Reference 64. For the RunIIb data, a similar event-by-event scale factor was used, 


\begin{tabular}{|c|c|c|}
\hline \multicolumn{2}{|c|}{ Lifetime Fit Parameters for RunIIa and RunIIb } \\
\hline \hline & RunIIa & RunIIb \\
\hline $\mathcal{F}_{\text {peak_bkg }}$ & $0.0142 \pm 0.0033$ & $0.1553 \pm 0.0022$ \\
\hline$\sigma_{\text {peak_bkg }}$ & $92.73 \pm 7.78 \mu \mathrm{m}$ & $100.14 \pm 1.36 \mu \mathrm{m}$ \\
\hline $\mathcal{F}_{0}$ & $0.1417 \pm 0.0039$ & $0.0 \pm 0.0046$ \\
\hline$s_{\text {bkg }}$ & $1.8256 \pm 0.0152$ & $2.2141 \pm 0.0251$ \\
\hline$c \tau_{\text {bkg }}$ & $642.94 \pm 2.82 \mu \mathrm{m}$ & $596.33 \pm 2.96 \mu \mathrm{m}$ \\
\hline $\mathcal{F}_{\text {peak }}$ & $0.0171 \pm 0.0024$ & $0.0245 \pm 0.0029$ \\
\hline $\mathcal{F}_{\text {tsens }}$ & $0.8654 \pm 0.1296$ & $0.6026 \pm 0.1342$ \\
\hline $\mathcal{F}_{\text {osc }}$ & $0.3273 \pm 0.0784$ & $0.3471 \pm 0.1369$ \\
\hline$c \tau_{B_{s}}$ & $456.54 \pm 3.79 \mu \mathrm{m}$ & $452.06 \pm 3.99 \mu \mathrm{m}$ \\
\hline
\end{tabular}

Table 3.5. Lifetime fit parameters for RunIIa and RunIIb

but grouped tracks into 16 categories, as opposed to the nearly 400 categories used in the RunIIa event-by-event scale factor.

The event-by-event scale factors were applied during the final signal selection. If the scale factors correctly scaled the VPDL for each event, the width of a pull distribution of $J / \psi \rightarrow \mu^{+} \mu^{-}$events should be exactly one. This would indicate that the now scaled uncertainties assigned to the vertex coordinates were correct. The pull distributions widths were checked and used for the estimation of systematic uncertainty on the decay length. The pull distributions, as well as more information on the systematic uncertainty is described in Section 4.1.2.

\subsection{Lifetime and Asymmetry}

The total tagged data sample was used to determine the following parameters, summarized in Table 3.5 in RunIIa and RunIIb.

Distributions of event VPDL are shown in Figures 3.45 and 3.46 using the optimal fit parameters in the fitting function, for RunIIa and RunIIb respectively. 

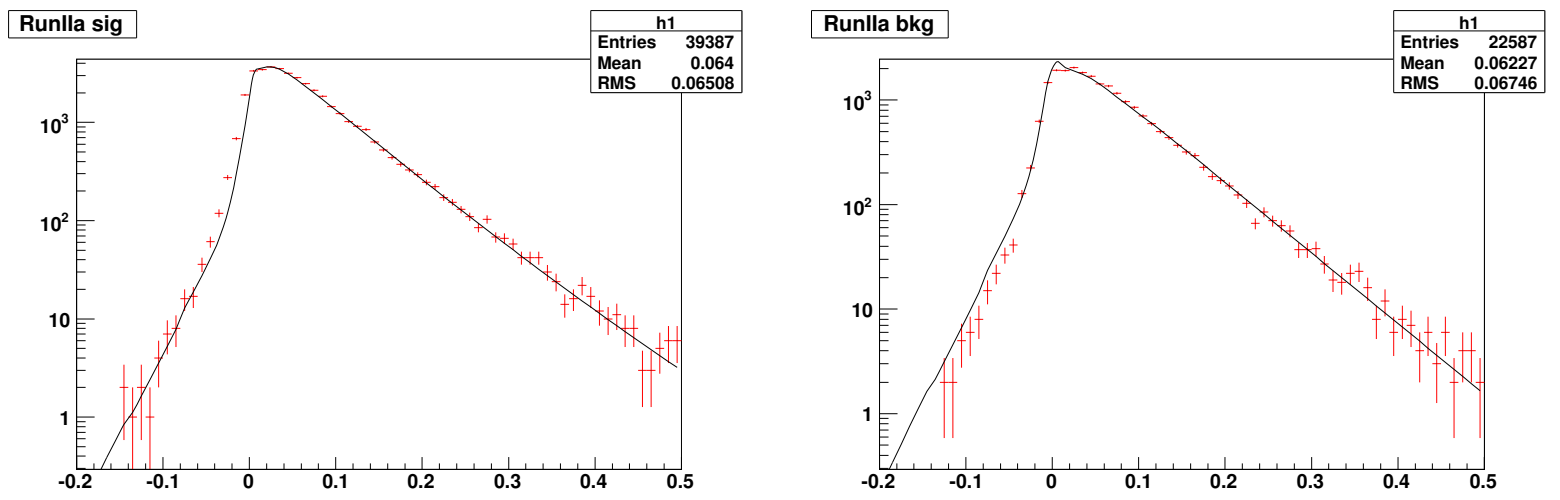

Figure 3.45. Distribution of RunIIa events on VPDL in signal peak region $\left(1.92<M_{D_{s}}<2.02 \mathrm{GeV}\right)$ (left) and sidebands $\left(1.72<M_{D_{s}}<1.77 \mathrm{GeV}\right.$ and $2.17<M_{D_{s}}<2.22 \mathrm{GeV}$ ) (right). Points represent experimental data; the line is the fitting function.
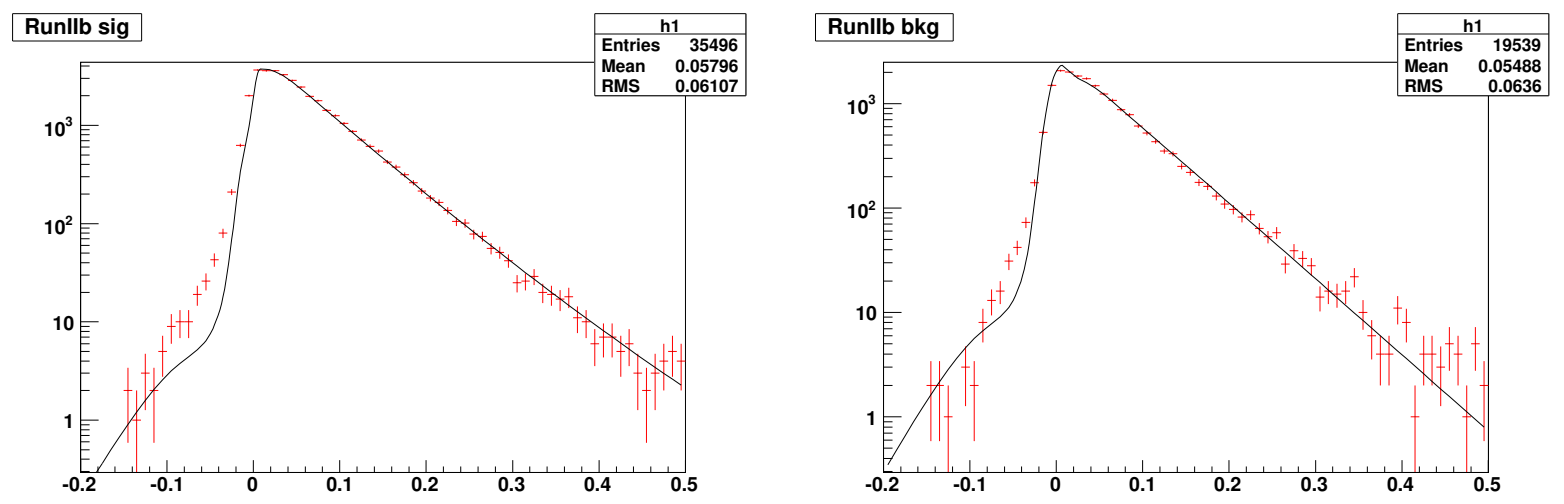

Figure 3.46. Distribution of RunIIb events on VPDL in signal peak region $\left(1.92<M_{D_{s}}<2.02 \mathrm{GeV}\right)$ (left) and sidebands $\left(1.72<M_{D_{s}}<1.77 \mathrm{GeV}\right.$ and $2.17<M_{D_{s}}<2.22 \mathrm{GeV}$ ) (right). Points represent experimental data; the line is the fitting function.

\subsection{Fit for $\Delta m_{s}$}

The final step in the analysis is to perform fits of the $B_{s}^{0}$ oscillation frequency, $\Delta m_{s}$. The standard method for setting limits on a mixing frequency is called the amplitude fit method [65]. The VPDL distribution of a given $B_{q}$ hadron, where $q=d, s$, is given by 
the equation:

$$
\begin{aligned}
& p_{s}^{\text {nos }}(x)=\frac{K}{c \tau_{B_{q}}} \exp \left(-\frac{K x}{c \tau_{B_{q}}}\right) \cdot 0.5 \cdot\left(1+\mathcal{A} \cdot \mathcal{D} \cos \left(\Delta m_{q} \cdot K x / c\right)\right) \\
& p_{s}^{o s c}(x)=\frac{K}{c \tau_{B_{q}}} \exp \left(-\frac{K x}{c \tau_{B_{q}}}\right) \cdot 0.5 \cdot\left(1-\mathcal{A} \cdot \mathcal{D} \cos \left(\Delta m_{q} \cdot K x / c\right)\right)
\end{aligned}
$$

where $\tau_{B_{q}}$ is the lifetime of the $B_{q}$ meson and $K$ is the $K$ factor. A fit parameter, $\mathcal{A}$, is added to the probability equation as shown above.

\subsubsection{The Amplitude Method}

For a given input value of $\Delta m_{s}$, we fit for $\mathcal{A}$. The values of $\mathcal{A}$ obtained from the fit are plotted as a function of oscillation frequency $\Delta m_{s}$. For the "true" value of the $B_{s}^{0}$ oscillation frequency, the amplitude $\mathcal{A}$ should be equal to 1 . The amplitude should give zero for any non- "true" value of $\Delta m_{s}$. If a scan across values of $\Delta m_{s}$ does not give an amplitude peak at 1 , then a lower limit on $\Delta m_{s}$ can easily be set.

The limit on $\Delta m_{s}$ is determined from calculating the likelihood that a fitted value of the amplitude could fluctuate to $\mathcal{A}=1$. The limit is the lowest value of $\Delta m_{s}$ that gives $\mathcal{A}_{d m s}+1.645 \sigma_{d m s}=1$. In addition, a value of "sensitivity" can be set using the amplitude method. The sensitivity is based on the probability that for a non- "true" value of $\Delta m_{s}$, the amplitude could fluctuate to $\mathcal{A}=1$. The sensitivity is set at the lowest value of $\Delta m_{s}$ for which $1.645 \sigma_{\Delta m_{s}}=1$, resulting in a $95 \%$ CL, where $\sigma_{\Delta m_{s}}$ is the uncertainty on the value of $\mathcal{A}$ at the point $\Delta m_{s}$. The method is able to probe the range of $\Delta m_{s}$ frequencies up to the sensitivity, but can make no claims about frequency values above the determined sensitivity. 
The ability to set a lower limit on $\Delta m_{s}$ and the ease with which results from many analyses can be combined for world averages are the two main advantages of using the amplitude method. However, since the method was created mainly for the purposes of setting limits, it is not the preferred method for determining a measured value of an oscillation frequency. For this, the standard is to use a log-likelihood scan of $\Delta m_{s}$.

\subsubsection{Negative Log-likelihood Determination of $\Delta m_{s}$}

The second method used for fitting for $\Delta m_{s}$ is a negative log-likelihood scan of the oscillation frequency. This can be done in two ways. First, the amplitude values obtained for each $\Delta m_{s}$ value in the above method can be converted into log-likelihood values. Second, the log-likelihood can be computed directly for each $\Delta m_{s}$ by fitting for log-likelihood rather than for amplitude. The first technique has been used for this analysis. The amplitude is converted into a log-likelihood value through the equation:

$$
-\Delta \log \mathcal{L}=\frac{1-2 \mathcal{A}}{2 \sigma_{\mathcal{A}}^{2}}
$$

By plotting the negative log-likelihood values versus $\Delta m_{s}$ and fitting for the minimum, we obtain the measured value of $\Delta m_{s}$. The significance of the result can be determined from the depth of the log-likelihood. Similarly to the amplitude method, the sensitivity of the log-likelihood is determined by the range of $\Delta m_{s}$ for which the log-likelihood has a parabolic minimum. The analysis has no sensitivity to $\Delta m_{s}$ values at and above the point at which the log-likelihood flattens. 
As a reminder, the previous result [17 of this analysis obtained a $95 \%$ confidence level

limit of $\Delta m_{s}=14.8 \mathrm{ps}^{-1}$ and a sensitivity of $14.1 \mathrm{ps}^{-1}$, with statistical and systematic errors included. 


\section{CHAPTER 4}

\section{Results}

An amplitude scan was performed on both the RunIIa and RunIIb data, and the results are shown in Figures 4.1 and 4.2 with statistical errors only. These results have greatly improved upon the previous work [17, as we have included the improved $K$ factors and sample compositions which both have the muon trigger turn-on curves applied, used the most recent PDG results for the $D_{s}^{-} D_{s}^{+}$branching fraction as input, have separate contributions from $B_{s}^{0} \rightarrow D_{s}^{-} D$ and $\bar{B}_{s}^{0} \rightarrow D_{s}^{-} D$, updated the MC version used for the RunIIa efficiencies as well as adding $\tau$ efficiencies for both run periods, and added more data. Additionally, the $K$ factor distributions are based on much higher MC statistics, allowing finer binning in both $K$ factor and $m\left(D_{s}^{-} \mu\right)$. The initial-state flavor-tagging has been greatly improved with the implementation of the SST and Event Charge taggers, as well as the separate dilution calibration for the $B^{+}$component. Finally, the decay length resolution understanding has been improved by the creation and implementation of event-by-event scale factors for both the RunIIa and RunIIb data.

Figure 4.3 shows the dependence of the parameter $\mathcal{A}$ and its error on the $\Delta m_{s}$ for the RunIIa data. A $95 \%$ confidence level limit on the oscillation frequency $\Delta m_{s}>14.4 \mathrm{ps}^{-1}$ and sensitivity $18.7 \mathrm{ps}^{-1}$ were obtained with statistical and systematic errors included. Figure 4.4 shows the dependence of the parameter $\mathcal{A}$ and its error on the $\Delta m_{s}$ for the RunIIb data. A $95 \%$ confidence level limit on the oscillation frequency $\Delta m_{s}>16.0 \mathrm{ps}^{-1}$ 
and sensitivity $20.3 \mathrm{ps}^{-1}$ were obtained with statistical and systematic errors included. A detailed description of the systematic uncertainty studies is given below in Section 4.1

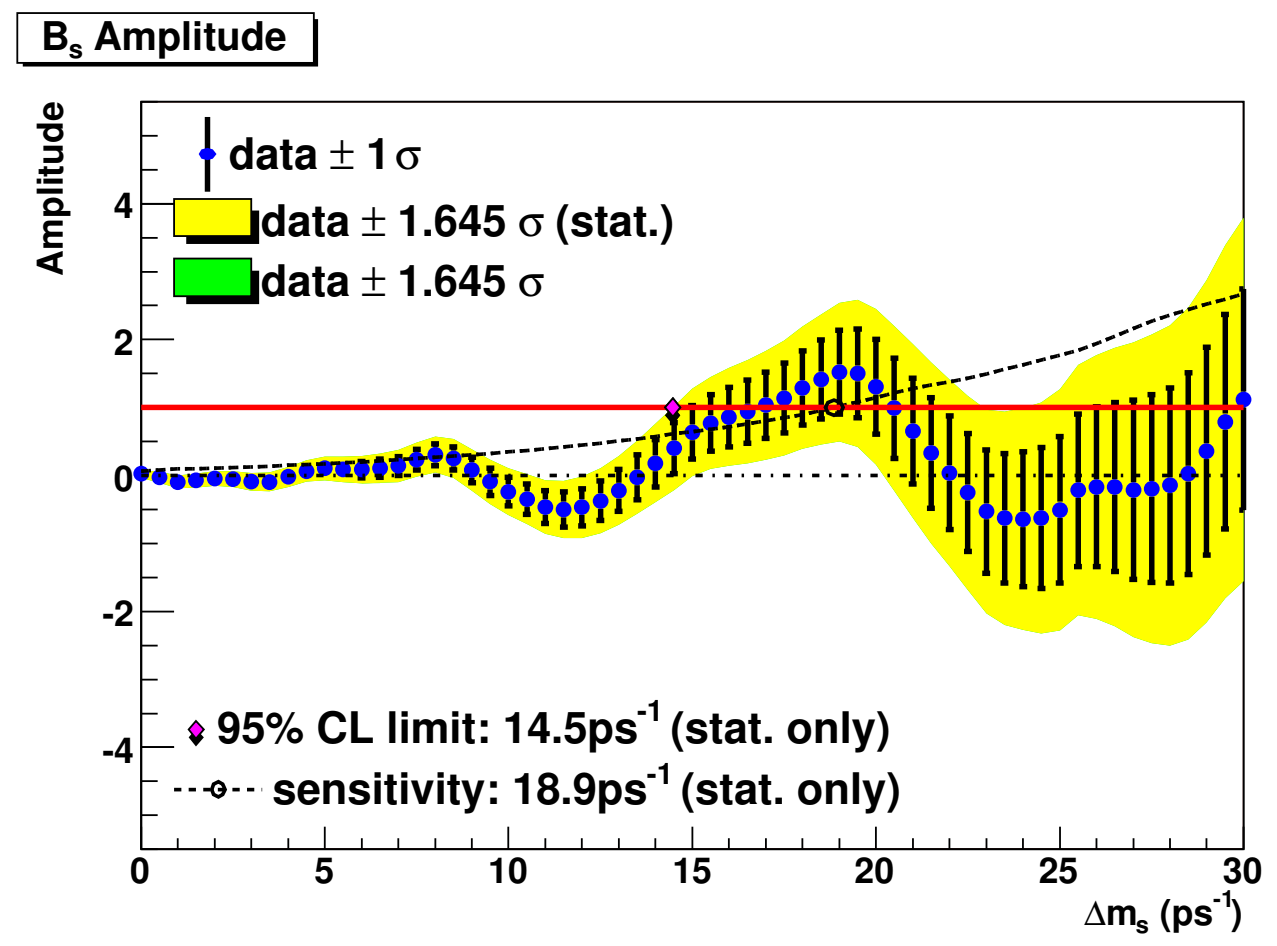

Figure 4.1. $\quad B_{s}^{0}$ oscillation amplitude with statistical errors for RunIIa data.

\subsubsection{Combined Result}

We computed a weighted average of the RunIIa and RunIIb results to get a single final result. The amplitude scan for the combination, with statistical errors only, is shown in Figure 4.5. We obtained a 95\% confidence level limit on the oscillation frequency $\Delta m_{s}>15.7 \mathrm{ps}^{-1}$ and a sensitivity of $23.2 \mathrm{ps}^{-1}$. The final combined result, with statistical and systematic uncertainties included, is shown in Figure 4.6. The limit obtained is $\Delta m_{s}=23.2 \mathrm{ps}^{-1}$ with a sensitivity of $\Delta m_{s}=23.0 \mathrm{ps}^{-1}$. 


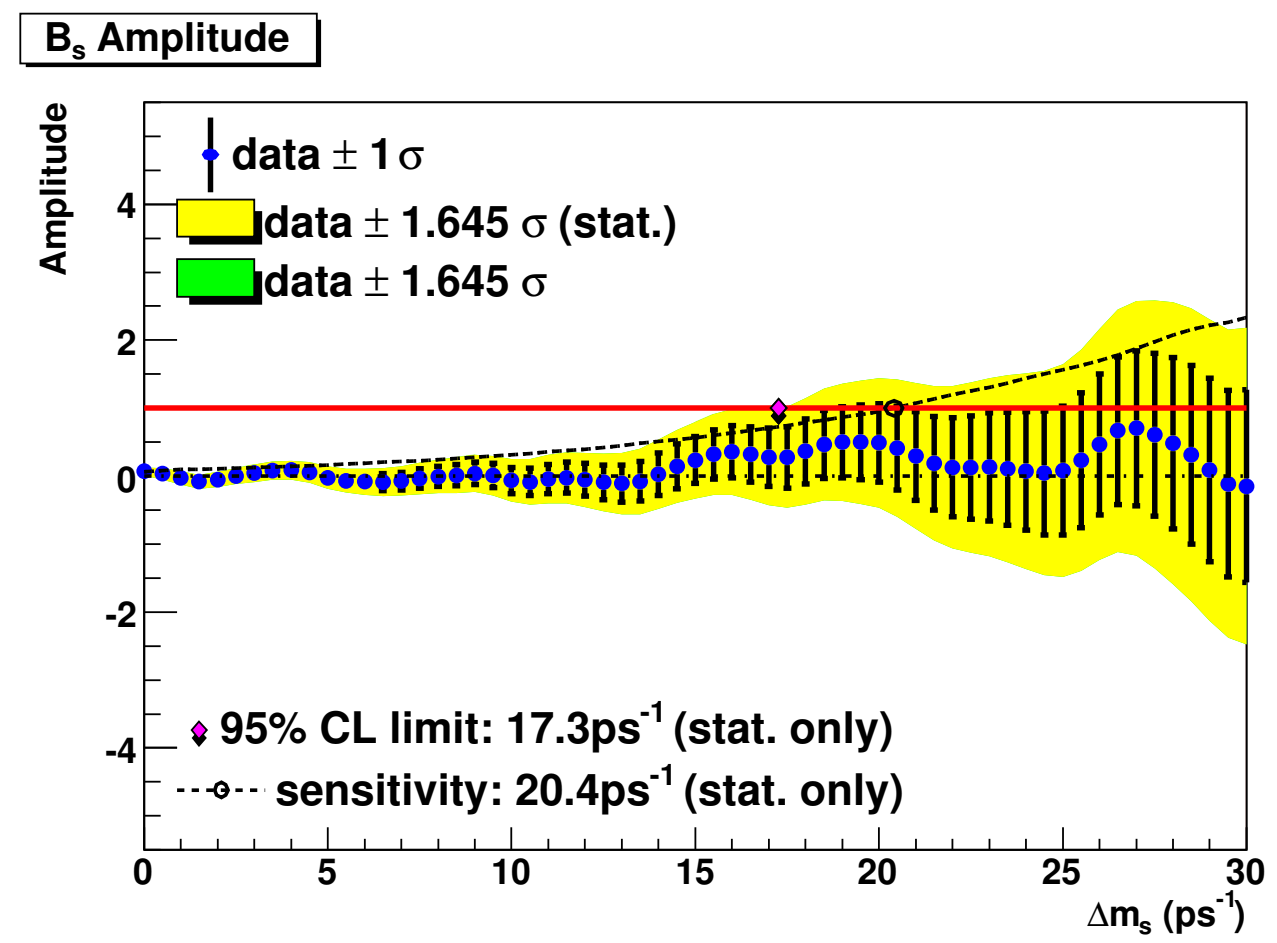

Figure 4.2. $\quad B_{s}^{0}$ oscillation amplitude with statistical errors for RunIIb data.

Figure 4.7 shows the dependence of the likelihood $\mathcal{L}$ (Eq. 3.12) on $\Delta m_{s}$ with the assumption that the amplitude $\mathcal{A}=1$, using RunIIa and RunIIb data. The minimum is at $\Delta m_{s}=18.86 \mathrm{ps}^{-1}$.

\subsection{Systematic Uncertainties}

We expect the following to contribute to the systematic uncertainty of the limit:

- Dilution

- Resolution

- Trigger Efficiency

- Sample composition

- $K$ factor 


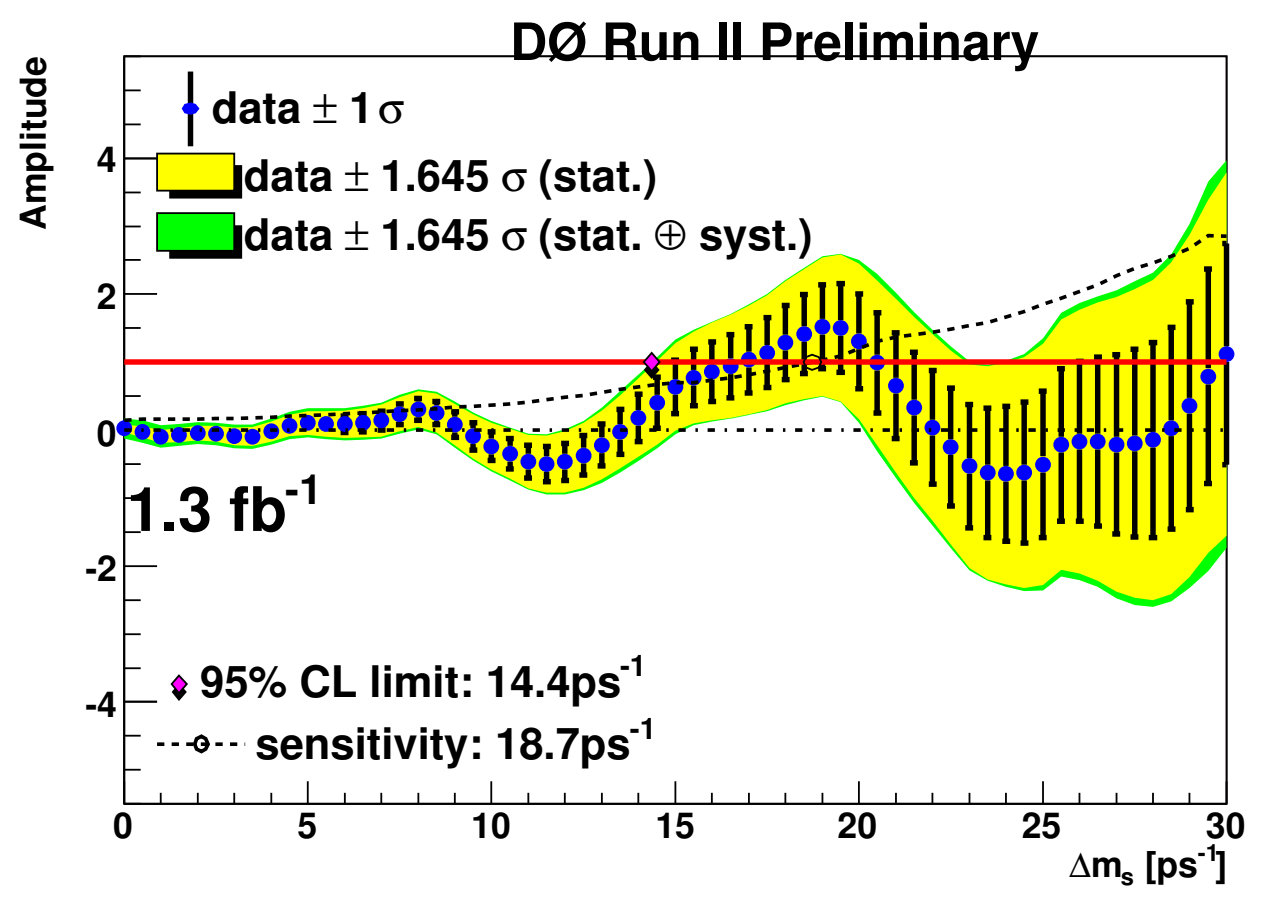

Figure 4.3. $\quad B_{s}^{0}$ oscillation amplitude with statistical and systematic errors for RunIIa data.

- Efficiency

In addition, we vary all of the parameters from the lifetime fit within errors, take into account the statistical fluctuation of the number of $D_{s}^{-}$candidates, and vary the reflection candidates by $100 \%$, or $0 \%$ to $1.4 \%$ of the $B_{s}^{0}$ candidates. A $1.4 \%$ reflection contribution nearly reaches the $1.5 \%$ maximum reflection contribution found in the study in Reference [66].

The contribution to the systematic error from the uncertainties of the input fit parameters can be estimated using the formula [65]:

$$
\sigma_{\mathcal{A}}^{\text {sys }}=\Delta \mathcal{A}+(1-\mathcal{A}) \frac{\Delta \sigma_{\mathcal{A}}}{\sigma_{\mathcal{A}}}
$$




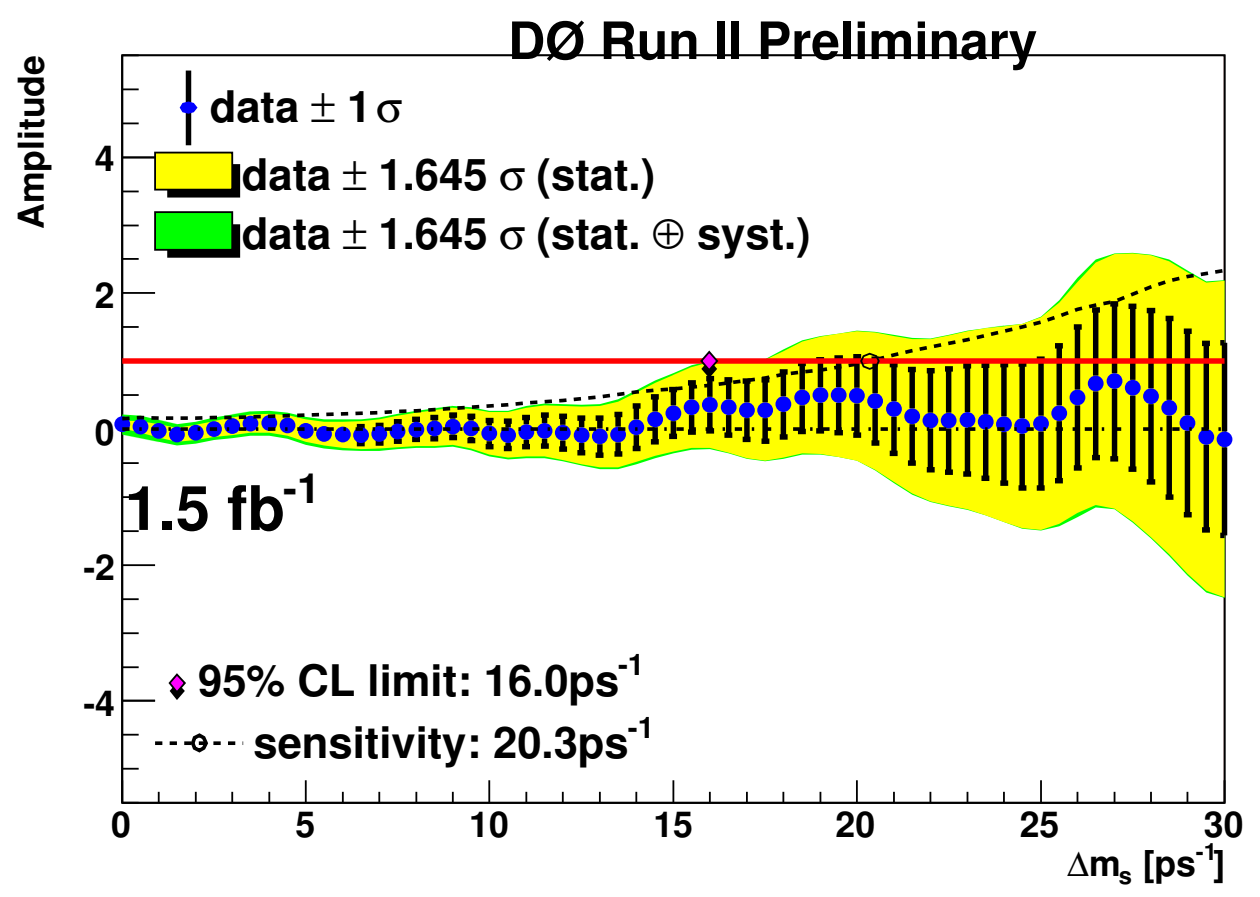

Figure 4.4. $\quad B_{s}^{0}$ oscillation amplitude with statistical and systematic errors for RunIIb data.

\subsubsection{Dilution}

The systematic uncertainty due to the dilution calibrations were taken from the $\pm 1 \sigma$ curves in Figure 4.8 for the $B_{s}^{0}$ dilution calibration. The $B^{+}$dilution calibration systematic uncertainty is taken from the $\pm 1 \sigma$ curves shown in Figure 4.9. The default $B^{+}$dilution calibration is taken from the p17 MC sample, and the systematics curves are chosen to cover the spread between the MC and data samples shown. 


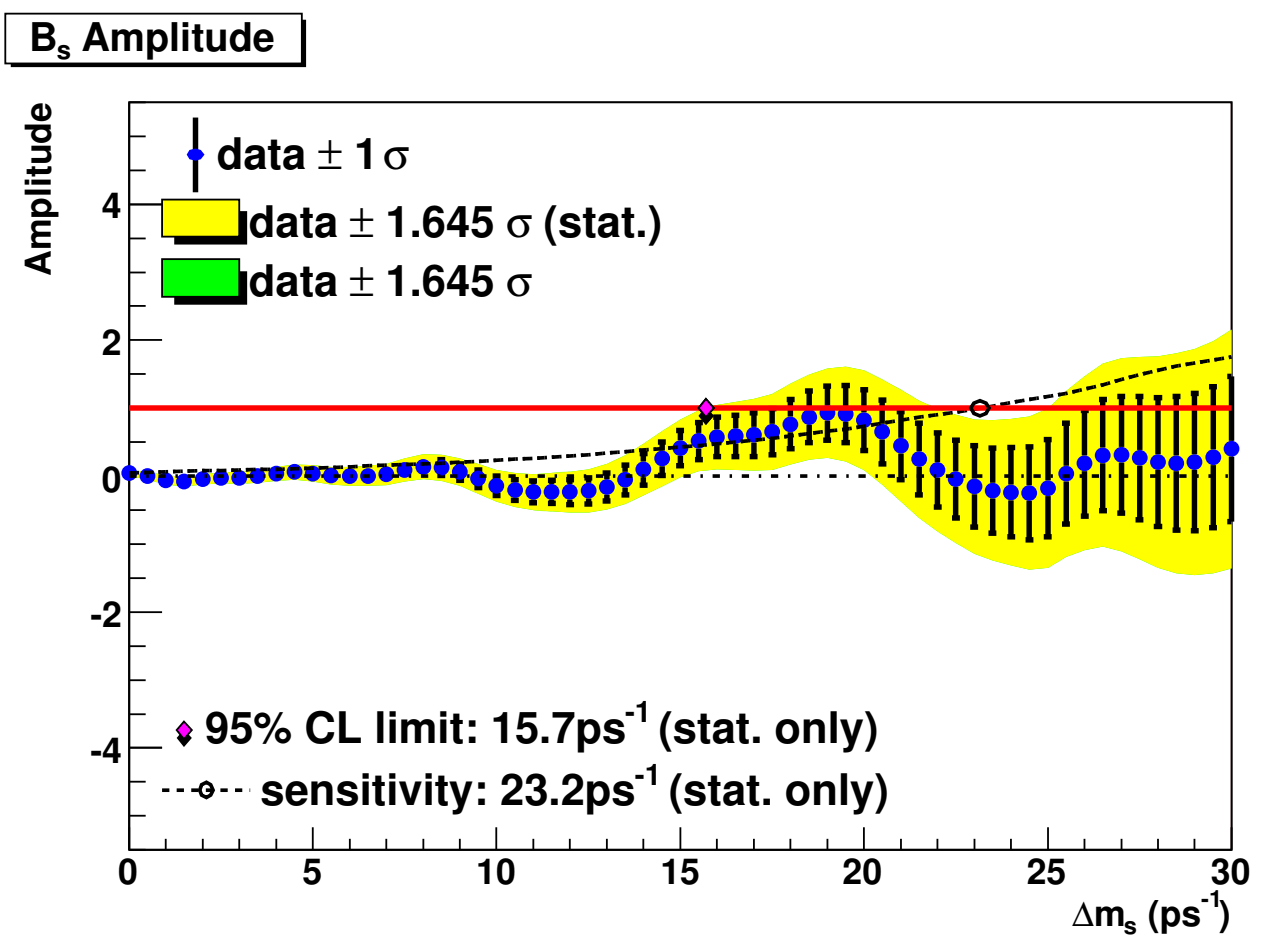

Figure 4.5. $\quad B_{s}^{0}$ oscillation amplitude with statistical errors for combined RunIIa and RunIIb data.

The calibration curve used for the $B_{s}^{0}$ dilution systematic was:

$$
\begin{array}{r}
\mathcal{D}\left(d_{p r}+1 \sigma\right)=45.28 \cdot\left|d_{p r}\right|^{2}+80.03 \cdot\left|d_{p r}\right|,\left|d_{p r}\right| \leq 0.6, \\
\mathcal{D}\left(d_{p r}+1 \sigma\right)=66.47 \cdot\left|d_{p r}\right|,\left|d_{p r}\right|>0.6 ; \\
\mathcal{D}\left(d_{p r}-1 \sigma\right)=22.52 \cdot\left|d_{p r}\right|^{2}+77.87 \cdot\left|d_{p r}\right|,\left|d_{p r}\right| \leq 0.6, \\
\mathcal{D}\left(d_{p r}+1 \sigma\right)=54.83 \cdot\left|d_{p r}\right|,\left|d_{p r}\right|>0.6 .
\end{array}
$$




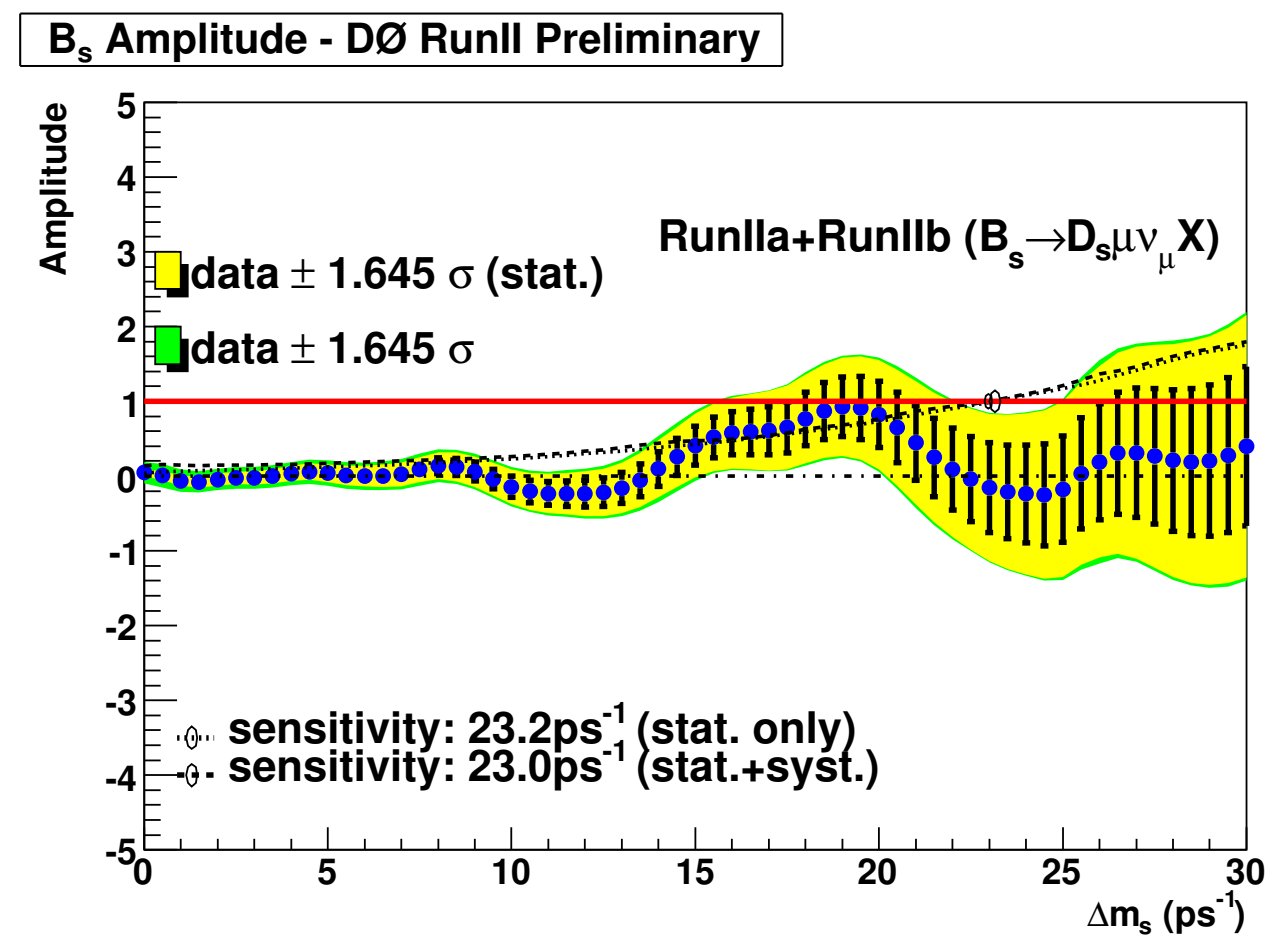

Figure 4.6. $\quad B_{s}^{0}$ oscillation amplitude with statistical and systematic errors for combined RunIIa and RunIIb data.

The calibration curve used for the $B^{+}$dilution systematic was:

$$
\begin{aligned}
& \mathcal{D}_{B_{u}}\left(d_{p r}+1 \sigma\right)=54.80 \cdot\left|d_{p r}\right|,\left|d_{p r}\right| \geq 0.45 \\
& \mathcal{D}_{B_{u}}\left(d_{p r}-1 \sigma\right)=33.06 \cdot\left|d_{p r}\right|,\left|d_{p r}\right| \geq 0.45
\end{aligned}
$$

\subsubsection{Resolution}

For RunIIa data, a pull plot was produced using $J / \psi$ data. The negative tail of the distribution was fitted with a double-gaussian, as shown in the left plot of Figure 4.10. The systematic error for the resolution was calculated by using the two scale factors from 


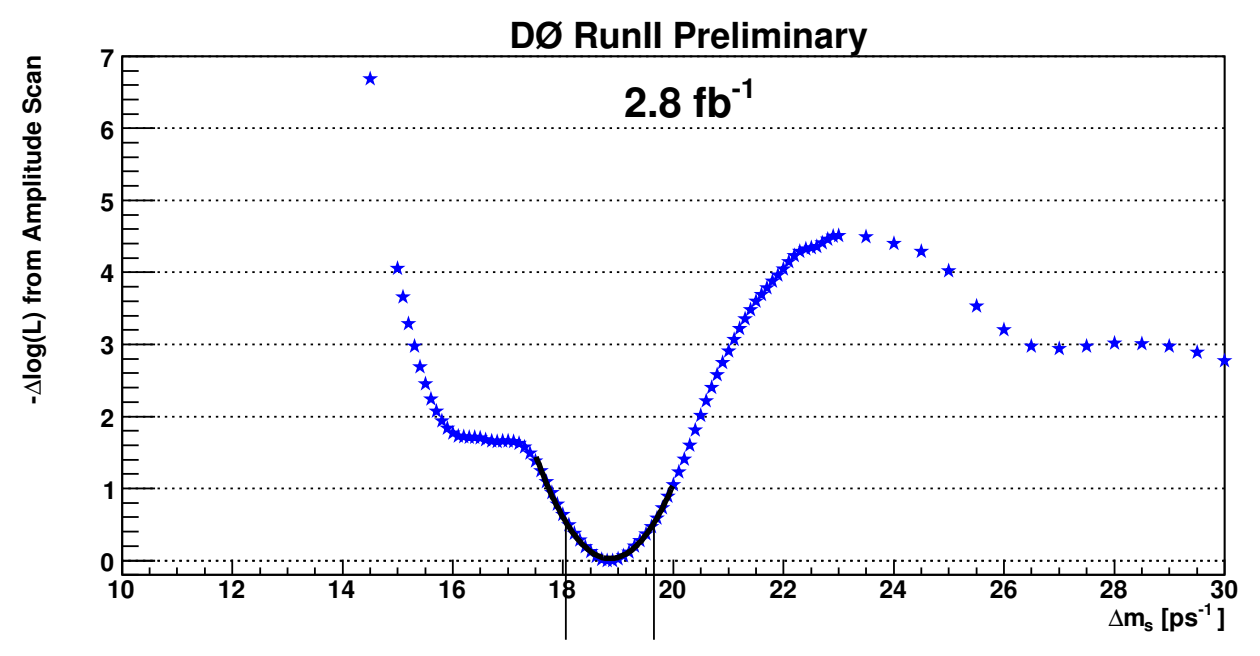

Figure 4.7. Negative log-likelihood versus $\Delta m_{s}$ with statistical errors only for combined RunIIa and RunIIb data.

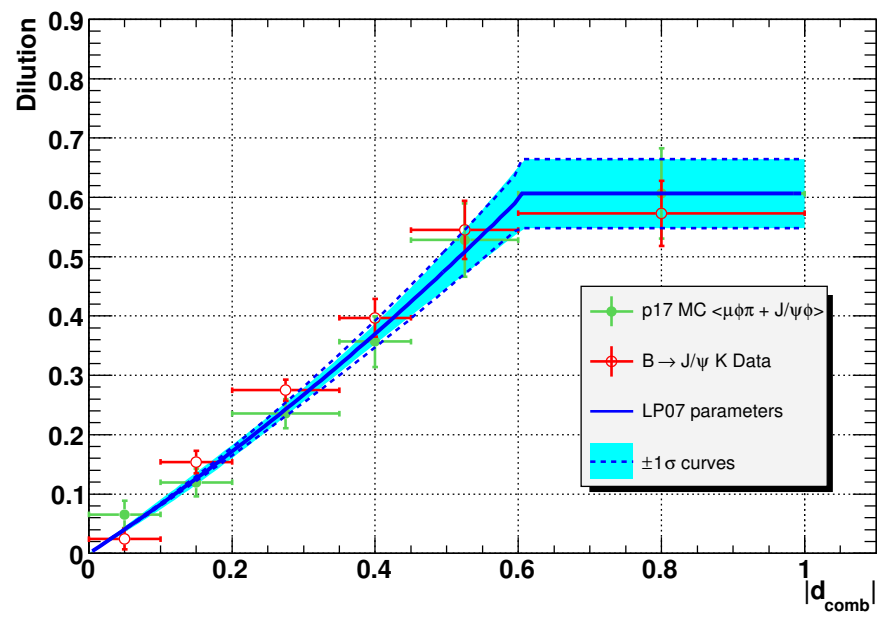

Figure 4.8. The systematic curves for the $B_{s}^{0}$ dilution calibration, indicated by the dashed-blue lines with the default calibration as the solid blue line.

the $J / \psi$ pull distribution in the fit, instead of the default single scale factor of 1 (since we use event-by-event scale factors in RunIIa).

For RunIIb data, the systematic uncertainty due to the resolution was calculated in a similar way. Again, a $J / \psi$ pull distribution was produced and the negative tail fit with 


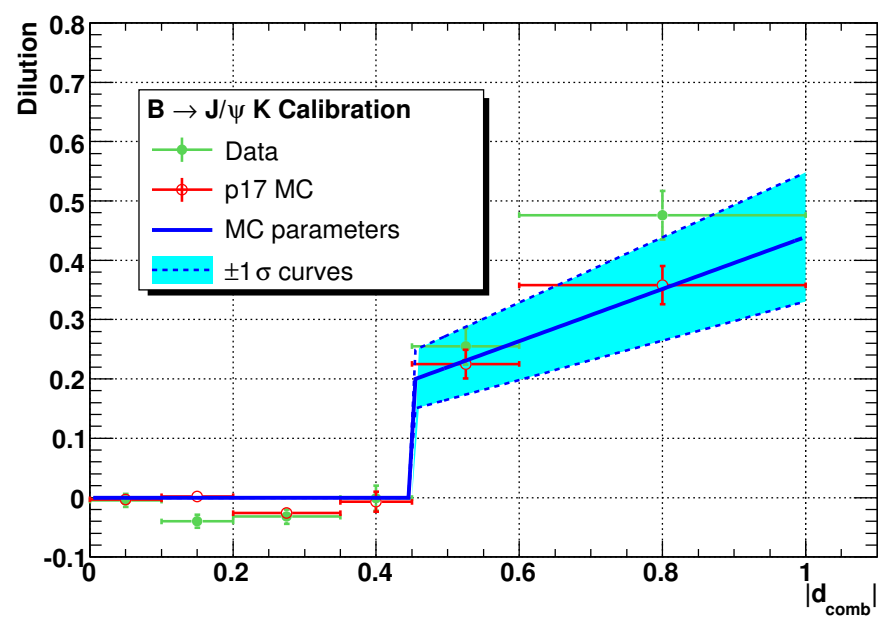

Figure 4.9. The $\pm 1 \sigma$ curves for the $B_{u}$ dilution systematic are indicated by the dashed-blue lines with the default calibration as the solid blue line.

a double-gaussian, as shown in the right plot of Figure 4.10. The systematic uncertainty for the resolution was calculated by using the two resulting scale factors in the fit, instead of a single scale factor of 1 .

\subsubsection{Sample Composition}

In addition to the variations in sample composition due to the trigger efficiency curves, the signal $\mu^{+} D_{s}^{-}$contribution was varied within its uncertainty. As mentioned in Section 3.6.3. varying the signal branching fraction within errors causes the maximum deviation in the sample composition, for most contributions. For the systematic, the signal contribution was varied from the default value of $7.9 \%$ to $5.5 \%$. 

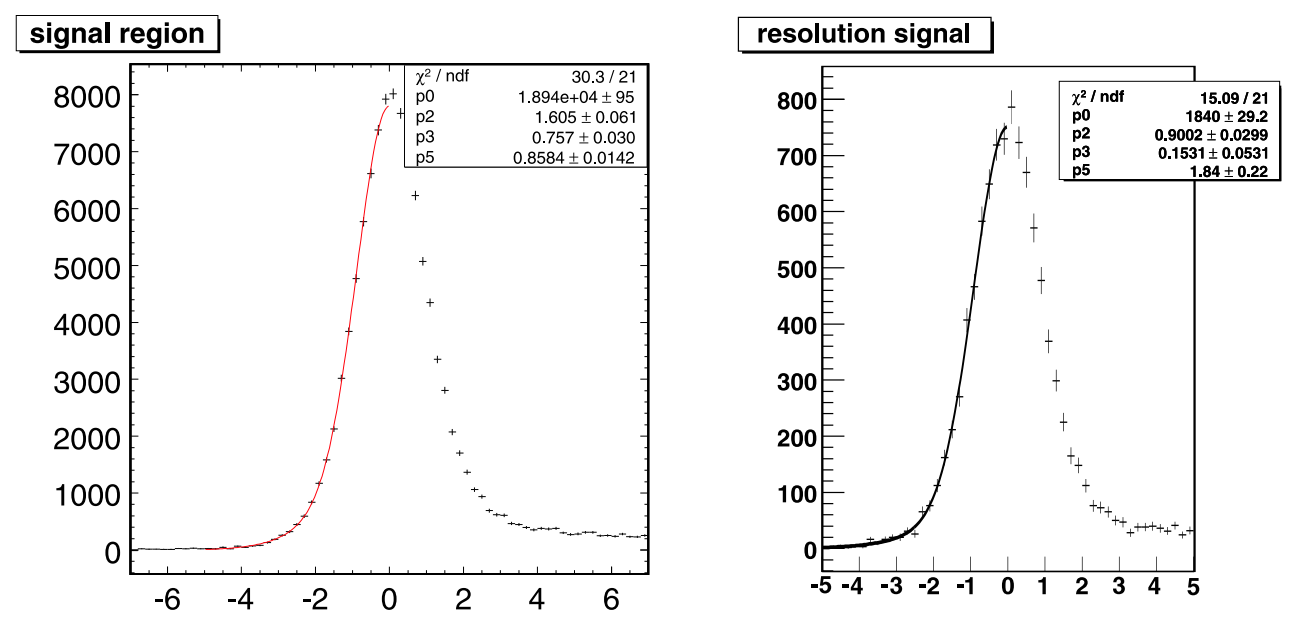

Figure 4.10. Pull of $J / \psi$ vertex distribution with respect to the primary vertex: $V P D L_{J / \psi} / \sigma_{P D L_{J / \psi}}$ for RunIIa on the left and RunIIb on the right, after the application of the event-by-event scale factors. For RunIIa, the fit of two gaussians to the negative tail of the pull distribution gives two resolution scale factors, $S F=0.8584 \pm 0.0142$ for $75.7 \%$ of the events and $S F=1.605 \pm 0.061$ for the remainder; for RunIIb, a similar fit gives $S F=0.9002 \pm 0.0299$ for $84.7 \%$ of the events and $S F=1.84 \pm 0.22$ for the remainder.

\subsubsection{K Factor}

The $K$ factor systematic due to the variation in the trigger efficiency curves has been discussed above. Additionally, a $\pm 2 \%$ variation in the $K$ factor was computed and used as systematic input. The choice of a $2 \%$ variation originated from studies done by Guennadi Borissov, Sergey Burdin, and Andrei Nomerotski [67]. For this analysis in particular, there are three possible sources (in addition to the trigger efficiency) of systematic uncertainty for the $K$ factors, as follows:

- momenta differences using generator-level information versus reconstructed momentum $(1.2 \%)$ 62],

- dependence of the $K$ factor on the $B$ momentum (2\% in Ref. [67]), and 
- using EvtGen values for relative $B_{s}^{0} \rightarrow \mu^{+} D_{s}^{-}$signal decays where PDG values are unavailable (2.1\% as the worst-case).

Wendy Taylor has performed a number of studies regarding the $K$ factor systematic uncertainties. The $2 \%$ variation attributed to the $K$ factor dependence on the $B$ momentum is now considered conservative, because $K$ factors are now binned in $m\left(\mu^{+} D_{s}^{-}\right)$and it has been shown that there is a flat dependence of the $K$ factor on $p_{T}\left(\mu^{+} D_{s}^{-}\right)$. Additionally, while the studies in Reference [62] showed that differences in RMS values of the $K$ factor distributions can lead to variations in the $K$ factor of greater than $2 \%$, it has been shown [68] that this does not impact the resolution of the $B_{s}^{0}$ transverse momentum.

\subsubsection{Trigger Efficiency}

A systematic is included to account for the variation in trigger efficiency curves that were applied to the sample composition and $K$ factor distributions. Figure 3.26 shows all of the possible efficiency curves for RunIIa and RunIIb. As mentioned previously, the central curve of each distribution was used as the default.

For the systematic, the uppermost and lowermost trigger efficiency curves were used as inputs to the sample composition and $K$ factor distributions to create two additional sets of distributions; one for the uppermost efficiency curve and one for the lower most. The $\Delta m_{s}$ fit was redone using these variations in sample composition $K$ factors. The resulting systematics are included in the final result. 


\subsubsection{Efficiency}

To check for a possible bias of the $\Delta m_{s}$ measurement due to the efficiency parameterization a study was performed with a p20 signal MC sample. The oscillation frequency was fixed to $19 \mathrm{ps}^{-1}$. First, we removed all of the lifetime-biasing selection cuts so the efficiency is flat as a function of VPDL. The likelihood scan corresponding to this case is shown in Figure 4.11 as the blue dots. Next we apply the default efficiency parameterization (from MC after applying lifetime cuts) in our likelihood minimization functions on the events where no lifetime-biasing selection cuts were used. The corresponding likelihood scan is shown in red in Figure 4.11,

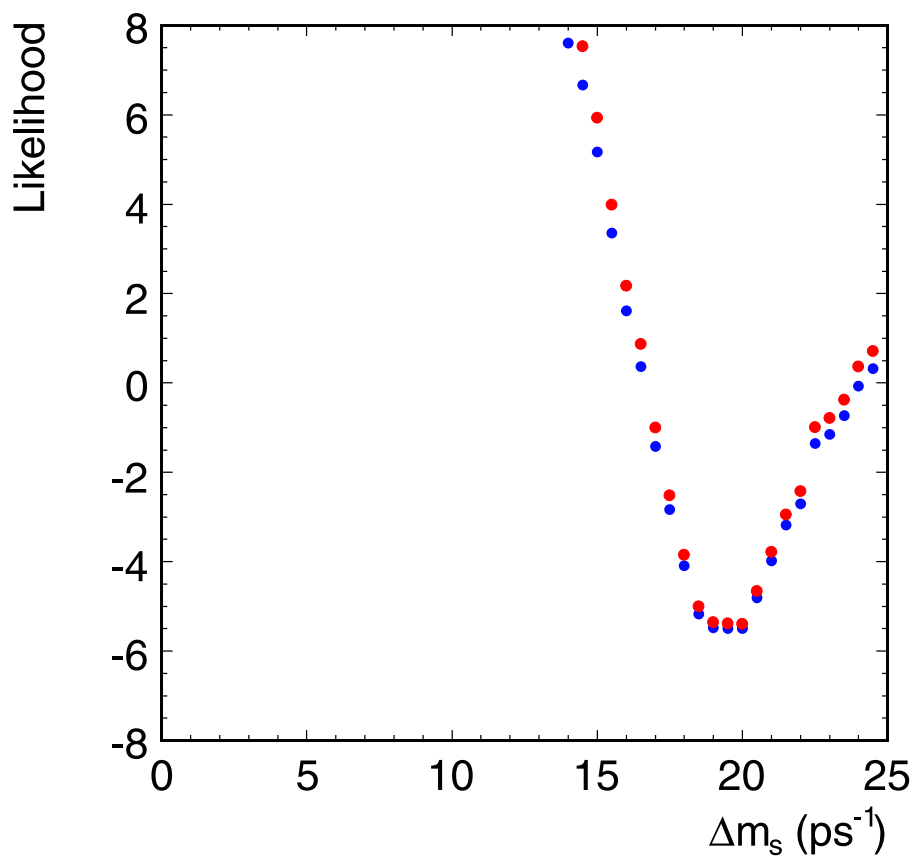

Figure 4.11. Likelihood scans using a flat efficiency function (blue) and the default efficiency (red) versus VPDL using events without lifetime biasing selection cuts. 
For an efficiency systematic, we take half of the difference between these two curves and use the relative error to translate to the data. We choose half of the difference as a flat efficiency with respect to VPDL is a very conservative assumption.

\subsubsection{Summary of Systematics}

Table 4.1 shows a list of the systematics and a short description of how each systematic was done. This can be used as a guide when reading the full systematics tables that follow. The ordering follows that of the combined RunIIa and RunIIb systematics table.

All studied contributions to the systematic uncertainty of the amplitude are listed in Table 4.2 for RunIIa, in Table 4.3 for RunIIb, and the combined systematics for RunIIa and RunIIb are listed in Table 4.4. The resulting systematic errors were obtained using the formula in Equation 4.1 and summed in quadrature. The result for RunIIa is shown in Figure 4.3, in Figure 4.4 for RunIIb, and the combined RunIIa and RunIIb $\Delta m_{s}$ scan with statistical and systematic errors is shown in Figure 4.5

Other potential sources of systematic uncertainty exist, but were not included in this analysis. One such source is that the detector could be mis-aligned by some small amount and that this mis-alignment could impact the result. It has been shown in previous DZero results 69 that a mis-alignment in the detector would cause, at most, a $2 \mu \mathrm{m}$ (about $0.007 \mathrm{ps}$, compared to the $1.43 \mathrm{ps} B_{s}^{0}$ lifetime) shift in the $B_{s}^{0}$ lifetime, which is a negligible contribution to the systematic uncertainty of this analysis. Another potential source is the impact of the trigger efficiency curve variations on the sample composition. It has been shown 1 that this causes a less than $0.1 \%$ variation in the sample composition, and

\footnotetext{
${ }^{1}$ Studies by Dmitri Tsybychev
} 


\begin{tabular}{|c|c|c|}
\hline \multicolumn{3}{|c|}{ Summary of Systematics for RunIIa and RunIIb } \\
\hline Systematic & Label in Tables & Description \\
\hline$B_{s}^{0}$ Lifetime uncertainty & $\mathrm{c} \tau_{B s}$ & Fitted lifetime value varied within error \\
\hline$B_{s}^{0}$ lifetime fixed to $439 \mu \mathrm{m}$ & $\mathrm{c} \tau_{B s}=439 \mu \mathrm{m}$ & Lifetime fixed to $438 \mu \mathrm{m}$ in fit \\
\hline Peaking background in signal & $\mathcal{F}_{\text {peak_sig }}$ & Varied within error \\
\hline Peaking fraction in bkg. & $\mathcal{F}_{\text {peak_bkg }}$ & Varied within error \\
\hline Width of peaking fraction in signal & $\sigma_{\text {peak_bkg }}$ & Varied within error \\
\hline Prompt fraction in bkg & $\mathcal{F}_{0}$ & Varied within error \\
\hline Background lifetime & $\mathrm{c} \tau_{b k g}$ & Varied within error \\
\hline Resolution SF in bkg & $s_{b k g}$ & Varied within error \\
\hline Fraction of mixing background & $\mathcal{F}_{\text {tsens }}$ & Varied within error \\
\hline Fraction of oscillating background & $\mathcal{F}_{\text {osc }}$ & Varied within error \\
\hline Statistical fluctuation of $D_{s}$ & $\Delta N_{D s}$ & Varied within error \\
\hline Number of reflection candidates & $\Delta N_{\text {refl }}$ & Varied by $100 \%$ \\
\hline$D_{s} D_{s}$ contribution & $B_{s}^{0} \rightarrow D_{s} D_{s}=6.8 \%$ & Contribution varied to $6.8 \%$ \\
\hline$D_{s} \mu$ contribution & $B_{s}^{0} \rightarrow D_{s} \mu \nu X=5.5 \%$ & Contribution varied to $5.5 \%$ \\
\hline Efficiency versus VPDL & $\epsilon$ versus VPDL & See section 4.1 .6 \\
\hline Resolution SF signal & $s f_{s i g}$ & See section 4.1 .2 \\
\hline$B_{s}^{0}$ Dilution uncertainty & $B_{s}$ dilution & See section 4.1.1 \\
\hline$B_{u}$ Dilution uncertainty & $B_{u}$ dilution & See section 4.1 .1 \\
\hline Non-zero $\Delta \Gamma / \Gamma$ & Non-zero $\Delta \Gamma / \Gamma$ & Set to $\pm 17.7 \mu \mathrm{m}$ of lifetime value \\
\hline Lifetime Parameter correlations & Parameter correlations & Accounts for correlation of background parameters \\
\hline K-factor variation $2 \%$ & K-factor variation $2 \%$ & See section 3.6 .2 \\
\hline K-factor Trigger Efficiency variations & K-factor trigger $\epsilon$ & See section 3.6 .2 \\
\hline
\end{tabular}

Table 4.1. Summary of the abbreviation of each systematic, and a short description of what was done. 
so has been neglected. Finally, the fitting code itself could bias a measurement of $\Delta m_{s}$; initial studies 2 show that the fit results are linear with respect to $\Delta m_{s}$, and any bias that exists is expected to be small.

\subsection{Cross-checks}

A number of cross-checks were performed to ensure the validity of the analysis. The most important of these are described in the following sections.

\subsubsection{Fit for $\Delta m_{d}$}

Because $\Delta m_{d}$ has been precisely measured [6], a fit of the $B_{d}^{0}$ oscillation frequency, $\Delta m_{d}$, is performed and serves as a cross-check for the method. An amplitude scan of the $B_{d}^{0}$ oscillation frequency that shows an amplitude of 1 at the known frequency of $0.5 \mathrm{ps}^{-1}$ indicates that the flavor-tagging dilution calibration is correct and the lifetime fits resulted in an optimal parameterization.

An amplitude $\Delta m_{d}$ scan was used for a cross-check of the dilution. This scan is more sensitive to a description of the combinatorial background and, therefore, the original description has been changed to take into account oscillations in the $B_{d}^{0}$ contribution. The resulting amplitude scan is shown in Figures 4.12 and 4.13 for RunIIa, and Figures 4.14 and 4.15 for RunIIb. The amplitude at $\Delta m_{d}=0.5 \mathrm{ps}^{-1}$ is in agreement with 1 , which confirms that the dilution calibration was performed correctly. This cross-check also shows the ability of the method to detect an oscillation signal.

\footnotetext{
${ }^{2}$ Initial MC studies by Hal Evans
} 
Table 4.2. Systematic uncertainties on the amplitude. The shifts of both the measured amplitude, $\Delta \mathcal{A}$, and its statistical uncertainty, $\Delta \sigma$, are listed for RunIIa.

\begin{tabular}{|c|c|c|c|c|c|c|c|c|c|c|c|c|}
\hline Osc. frequency & & $0.0 \mathrm{ps}^{-1}$ & $3.0 \mathrm{ps}^{-1}$ & $6.0 \mathrm{ps}^{-1}$ & $9.0 \mathrm{ps}^{-1}$ & $12.0 \mathrm{ps}^{-1}$ & $15.0 \mathrm{ps}^{-1}$ & $18.0 \mathrm{ps}^{-1}$ & $21.0 \mathrm{ps}^{-1}$ & $24.0 \mathrm{ps}^{-1}$ & $27.0 \mathrm{ps}^{-1}$ & $30.0 \mathrm{ps}^{-1}$ \\
\hline $\mathcal{A}$ & & 0.025 & -0.092 & 0.088 & 0.082 & -0.467 & 0.635 & 1.291 & 0.655 & -0.642 & -0.210 & 1.117 \\
\hline Stat. uncertainty & & 0.036 & 0.078 & 0.123 & 0.185 & 0.274 & 0.392 & 0.546 & 0.778 & 0.992 & 1.318 & 1.624 \\
\hline $\mathrm{c} \tau_{B s}$ & $\Delta \mathcal{A}$ & -0.000 & -0.000 & +0.000 & +0.000 & -0.001 & +0.003 & +0.004 & +0.000 & -0.004 & -0.002 & +0.001 \\
\hline & $\Delta \sigma$ & -0.000 & +0.000 & +0.000 & +0.000 & +0.001 & +0.001 & +0.001 & +0.002 & +0.002 & +0.004 & +0.001 \\
\hline $\mathrm{c} \tau_{B s}=439 \mu \mathrm{m}$ & $\Delta \mathcal{A}$ & +0.000 & +0.000 & -0.002 & +0.000 & +0.005 & -0.012 & -0.018 & -0.002 & +0.020 & +0.013 & -0.003 \\
\hline & $\Delta \sigma$ & +0.000 & -0.000 & -0.001 & -0.001 & -0.002 & -0.004 & -0.005 & -0.009 & -0.012 & -0.017 & -0.020 \\
\hline $\mathcal{F}_{\text {peak_sig }}$ & $\Delta \mathcal{A}$ & -0.000 & -0.000 & +0.000 & +0.000 & -0.005 & +0.004 & +0.010 & +0.001 & -0.022 & -0.022 & -0.002 \\
\hline & $\Delta \sigma$ & +0.000 & +0.000 & +0.000 & +0.001 & +0.001 & +0.002 & +0.003 & +0.006 & +0.009 & +0.014 & +0.019 \\
\hline $\mathcal{F}_{\text {peak_bkg }}$ & $\Delta \mathcal{A}$ & +0.000 & +0.000 & +0.000 & +0.000 & -0.001 & +0.001 & +0.002 & +0.001 & -0.002 & -0.000 & +0.003 \\
\hline & $\Delta \sigma$ & -0.000 & -0.000 & +0.000 & +0.000 & +0.000 & +0.000 & +0.000 & +0.001 & +0.001 & $\begin{array}{r}+0.002 \\
\end{array}$ & +0.002 \\
\hline$\sigma_{p e a k_{-} b k g}$ & $\Delta \mathcal{A}$ & +0.000 & -0.000 & -0.000 & -0.000 & +0.000 & +0.000 & -0.000 & -0.000 & +0.001 & +0.001 & -0.001 \\
\hline & $\Delta \sigma$ & +0.000 & -0.000 & +0.000 & +0.000 & -0.000 & -0.000 & -0.000 & -0.000 & -0.000 & -0.000 & -0.001 \\
\hline $\mathcal{F}^{0}$ & $\Delta \mathcal{A}$ & +0.000 & +0.000 & +0.000 & +0.000 & -0.001 & +0.001 & +0.003 & +0.002 & -0.003 & -0.002 & +0.003 \\
\hline & $\Delta \sigma$ & -0.000 & -0.000 & +0.000 & +0.000 & +0.000 & +0.000 & +0.001 & +0.001 & +0.002 & +0.003 & +0.003 \\
\hline $\mathrm{c} \tau_{b k g}$ & $\Delta \mathcal{A}$ & +0.000 & +0.000 & -0.000 & -0.000 & +0.000 & -0.000 & -0.001 & +0.000 & +0.000 & -0.000 & +0.001 \\
\hline & $\Delta \sigma$ & +0.000 & -0.000 & -0.000 & -0.000 & -0.000 & -0.000 & -0.000 & -0.000 & -0.000 & -0.001 & -0.001 \\
\hline$s f_{b k g}$ & $\Delta \mathcal{A}$ & +0.000 & -0.000 & -0.000 & -0.000 & -0.001 & -0.001 & -0.001 & -0.001 & -0.000 & -0.001 & -0.002 \\
\hline & $\Delta \sigma$ & +0.000 & -0.000 & -0.000 & -0.000 & -0.000 & -0.000 & -0.000 & -0.000 & -0.000 & -0.000 & -0.002 \\
\hline $\mathcal{F}_{\text {tsens }}$ & $\Delta \mathcal{A}$ & -0.013 & +0.007 & +0.003 & +0.003 & +0.000 & -0.002 & -0.004 & -0.007 & -0.009 & -0.020 & -0.025 \\
\hline & $\Delta \sigma$ & -0.000 & -0.000 & -0.000 & -0.000 & -0.000 & -0.000 & -0.000 & -0.000 & -0.000 & -0.000 & +0.000 \\
\hline $\mathcal{F}_{\text {OSC }}$ & $\begin{array}{l}\Delta \mathcal{A} \\
\Delta \sigma\end{array}$ & $\begin{array}{l}-0.004 \\
-0.000\end{array}$ & $\begin{array}{l}+0.006 \\
-0.000\end{array}$ & $\begin{array}{l}+0.002 \\
-0.000\end{array}$ & $\begin{array}{l}0.001 \\
-0.000\end{array}$ & $\begin{array}{l}-0.000 \\
-0.000\end{array}$ & $\begin{array}{l}-0.003 \\
-0.000\end{array}$ & $\begin{array}{l}-0.006 \\
-0.000\end{array}$ & $\begin{array}{l}-0.011 \\
+0.000\end{array}$ & $\begin{array}{l}-0.013 \\
+0.000\end{array}$ & $\begin{array}{l}-0.021 \\
+0.000\end{array}$ & $\begin{array}{l}-0.022 \\
+0.011\end{array}$ \\
\hline$\Delta N_{D s}$ & $\Delta \mathcal{A}$ & -0.001 & +0.000 & +0.001 & +0.000 & -0.001 & +0.003 & +0.005 & +0.001 & -0.001 & -0.000 & +0.003 \\
\hline & $\Delta \sigma$ & +0.000 & +0.000 & +0.000 & +0.001 & +0.001 & +0.001 & +0.002 & +0.002 & +0.003 & +0.004 & +0.007 \\
\hline$\Delta N_{r e f l}$ & $\Delta \mathcal{A}$ & -0.002 & +0.001 & +0.002 & +0.001 & -0.001 & -0.000 & +0.004 & +0.007 & +0.006 & +0.003 & +0.004 \\
\hline & $\Delta \sigma$ & +0.000 & +0.000 & +0.000 & +0.000 & +0.001 & +0.001 & +0.001 & +0.002 & +0.002 & +0.003 & +0.004 \\
\hline $\operatorname{Br}\left(D_{s} D_{s}\right)=6.8 \%$ & $\Delta \mathcal{A}$ & +0.000 & -0.000 & +0.000 & +0.000 & -0.001 & +0.002 & +0.003 & +0.000 & -0.003 & -0.002 & +0.002 \\
\hline & $\Delta \sigma$ & +0.000 & +0.000 & +0.000 & +0.000 & +0.001 & +0.001 & +0.001 & +0.001 & +0.002 & +0.002 & +0.003 \\
\hline $\operatorname{Br}\left(D_{s} \mu X\right)=5.5 \%$ & $\Delta \mathcal{A}$ & +0.009 & -0.006 & +0.000 & -0.002 & -0.007 & +0.011 & +0.020 & +0.008 & -0.009 & -0.004 & +0.017 \\
\hline & $\Delta \sigma$ & +0.001 & +0.001 & +0.002 & +0.002 & +0.003 & +0.004 & +0.005 & +0.007 & +0.009 & +0.011 & +0.013 \\
\hline$\epsilon$ versus VPDL & $\Delta \mathcal{A}$ & 0.000 & 0.000 & 0.000 & 0.000 & 0.000 & 0.000 & 0.000 & 0.000 & 0.000 & 0.000 & 0.000 \\
\hline & $\Delta \sigma$ & -0.036 & -0.078 & -0.122 & -0.185 & -0.273 & -0.392 & -0.508 & -0.748 & -0.976 & -1.314 & -1.603 \\
\hline$s f_{s i g}$ & $\Delta \mathcal{A}$ & -0.000 & +0.001 & +0.000 & +0.005 & +0.011 & +0.004 & -0.001 & +0.065 & -0.104 & -0.348 & -0.394 \\
\hline & $\Delta \sigma$ & +0.000 & +0.001 & +0.002 & +0.003 & +0.002 & +0.001 & -0.006 & -0.022 & -0.038 & -0.061 & -0.075 \\
\hline$B_{s}$ & $\Delta \mathcal{A}$ & +0.004 & -0.009 & +0.009 & +0.008 & -0.030 & +0.043 & +0.086 & +0.046 & -0.039 & -0.037 & +0.061 \\
\hline dilution & $\Delta \sigma$ & +0.003 & +0.005 & +0.008 & +0.013 & +0.018 & +0.026 & +0.037 & +0.051 & +0.066 & +0.084 & +0.105 \\
\hline$B_{u}$ & $\Delta \mathcal{A}$ & +0.010 & -0.003 & -0.001 & -0.002 & -0.000 & +0.001 & +0.000 & +0.001 & +0.001 & +0.008 & +0.012 \\
\hline dilution & $\Delta \sigma$ & +0.000 & +0.000 & +0.000 & +0.000 & +0.000 & +0.000 & +0.000 & +0.000 & +0.000 & +0.000 & +0.000 \\
\hline Non-zero $\Delta \Gamma / \Gamma$ & $\Delta \mathcal{A}$ & +0.000 & +0.000 & -0.002 & +0.000 & +0.005 & -0.012 & -0.018 & -0.002 & +0.021 & +0.013 & -0.005 \\
\hline & $\Delta \sigma$ & +0.000 & -0.000 & -0.001 & -0.001 & -0.002 & -0.004 & -0.006 & -0.009 & -0.012 & -0.018 & -0.021 \\
\hline Parameter & $\Delta \mathcal{A}$ & +0.000 & -0.000 & +0.000 & +0.000 & -0.000 & +0.000 & +0.001 & +0.000 & -0.002 & -0.002 & -0.001 \\
\hline correlations & $\Delta \sigma$ & -0.000 & -0.000 & -0.000 & -0.000 & +0.000 & +0.000 & +0.000 & +0.000 & +0.001 & +0.001 & +0.001 \\
\hline K-factor variation & $\Delta \mathcal{A}$ & -0.000 & +0.003 & -0.003 & +0.067 & -0.027 & -0.131 & -0.102 & +0.286 & +0.006 & +0.035 & -0.456 \\
\hline $2 \%$ & $\Delta \sigma$ & -0.000 & -0.001 & -0.002 & -0.004 & -0.007 & -0.009 & -0.020 & -0.030 & -0.033 & -0.069 & -0.049 \\
\hline K-factor trigger $\epsilon$ & $\Delta \mathcal{A}$ & +0.000 & +0.000 & +0.001 & +0.000 & +0.001 & -0.005 & +0.007 & +0.000 & -0.006 & +0.032 & -0.028 \\
\hline uncertainty & $\Delta \sigma$ & +0.000 & +0.000 & +0.000 & +0.000 & +0.000 & +0.000 & +0.000 & +0.001 & +0.001 & +0.000 & +0.003 \\
\hline Total syst. & $\sigma_{\text {tot }}^{\text {sys }}$ & 0.081 & 0.069 & 0.077 & 0.089 & 0.100 & 0.157 & 0.123 & 0.289 & 0.189 & 0.410 & 0.601 \\
\hline Total & $\sigma_{t o t}$ & 0.088 & 0.105 & 0.145 & 0.205 & 0.292 & 0.422 & 0.560 & 0.829 & 1.010 & 1.380 & 1.732 \\
\hline
\end{tabular}


Table 4.3. Systematic uncertainties on the amplitude. The shifts of both the measured amplitude, $\Delta \mathcal{A}$, and its statistical uncertainty, $\Delta \sigma$, are listed for RunIIb.

\begin{tabular}{|c|c|c|c|c|c|c|c|c|c|c|c|c|}
\hline Osc. frequency & & $0.0 \mathrm{ps}^{-1}$ & $3.0 \mathrm{ps}^{-1}$ & $6.0 \mathrm{ps}^{-1}$ & $9.0 \mathrm{ps}^{-1}$ & $12.0 \mathrm{ps}^{-1}$ & $15.0 \mathrm{ps}^{-1}$ & $18.0 \mathrm{ps}^{-1}$ & $21.0 \mathrm{ps}^{-1}$ & $24.0 \mathrm{ps}^{-1}$ & $27.0 \mathrm{ps}^{-1}$ & $30.0 \mathrm{ps}^{-1}$ \\
\hline $\mathcal{A}$ & & 0.068 & 0.041 & -0.083 & 0.040 & -0.054 & 0.234 & 0.364 & 0.297 & 0.075 & 0.705 & -0.148 \\
\hline Stat. uncertainty & & 0.037 & 0.077 & 0.116 & 0.168 & 0.242 & 0.344 & 0.477 & 0.653 & 0.875 & 1.140 & 1.417 \\
\hline $\mathrm{c} \tau_{B s}$ & $\Delta \mathcal{A}$ & +0.000 & +0.000 & -0.001 & +0.000 & -0.000 & +0.001 & +0.001 & +0.000 & +0.001 & +0.004 & -0.001 \\
\hline & $\Delta \sigma$ & -0.000 & +0.000 & +0.000 & +0.000 & +0.001 & +0.001 & +0.001 & +0.002 & +0.003 & +0.004 & +0.005 \\
\hline $\mathrm{c} \tau_{B s}=439 \mu \mathrm{m}$ & $\Delta \mathcal{A}$ & +0.000 & -0.000 & +0.002 & -0.001 & +0.001 & -0.003 & -0.005 & -0.003 & -0.002 & -0.011 & +0.005 \\
\hline & $\Delta \sigma$ & +0.000 & -0.000 & -0.001 & -0.001 & -0.002 & -0.003 & -0.004 & -0.006 & -0.009 & -0.012 & -0.015 \\
\hline $\mathcal{F}_{\text {peak_sig }}$ & $\Delta \mathcal{A}$ & +0.000 & +0.000 & -0.001 & +0.000 & -0.002 & +0.001 & +0.001 & -0.003 & -0.011 & +0.002 & -0.026 \\
\hline & $\Delta \sigma$ & +0.000 & +0.000 & +0.000 & +0.001 & +0.002 & +0.003 & +0.004 & +0.007 & +0.011 & +0.016 & +0.021 \\
\hline $\mathcal{F}_{\text {peak_bkg }}$ & $\Delta \mathcal{A}$ & +0.000 & +0.000 & -0.000 & +0.000 & +0.000 & +0.001 & +0.001 & +0.001 & +0.002 & +0.004 & +0.002 \\
\hline & $\Delta \sigma$ & -0.000 & -0.000 & +0.000 & +0.000 & +0.000 & +0.000 & +0.000 & +0.001 & +0.001 & +0.001 & +0.002 \\
\hline$\sigma_{p e a k_{-} b k g}$ & $\Delta \mathcal{A}$ & +0.000 & -0.000 & -0.000 & +0.000 & +0.000 & -0.000 & -0.001 & -0.000 & +0.001 & -0.002 & +0.000 \\
\hline & $\Delta \sigma$ & +0.000 & -0.000 & 0.000 & -0.000 & -0.000 & -0.000 & -0.000 & -0.000 & -0.001 & -0.001 & -0.002 \\
\hline $\mathcal{F}^{0}$ & $\Delta \mathcal{A}$ & +0.000 & +0.000 & -0.000 & +0.000 & +0.000 & +0.002 & +0.003 & +0.004 & +0.004 & +0.011 & +0.006 \\
\hline & $\Delta \sigma$ & -0.000 & -0.000 & +0.000 & +0.000 & +0.000 & +0.000 & +0.001 & +0.002 & +0.003 & +0.004 & +0.006 \\
\hline $\mathrm{c} \tau_{b k g}$ & $\Delta \mathcal{A}$ & +0.000 & -0.000 & +0.000 & -0.000 & -0.000 & -0.000 & -0.000 & -0.000 & -0.000 & -0.000 & +0.000 \\
\hline & $\Delta \sigma$ & +0.000 & -0.000 & -0.000 & -0.000 & -0.000 & -0.000 & -0.000 & -0.000 & -0.000 & -0.001 & -0.001 \\
\hline$s f_{b k g}$ & $\Delta \mathcal{A}$ & +0.000 & +0.000 & -0.000 & -0.000 & -0.000 & -0.000 & -0.001 & -0.001 & -0.001 & -0.001 & -0.001 \\
\hline & $\Delta \sigma$ & -0.000 & -0.000 & -0.000 & -0.000 & -0.000 & -0.000 & -0.000 & -0.000 & -0.000 & -0.000 & 0.000 \\
\hline $\mathcal{F}_{\text {tsens }}$ & $\Delta \mathcal{A}$ & -0.014 & +0.006 & +0.003 & -0.003 & -0.005 & -0.004 & -0.004 & -0.011 & -0.024 & -0.024 & -0.022 \\
\hline & $\Delta \sigma$ & -0.000 & -0.000 & -0.000 & -0.000 & -0.000 & -0.000 & -0.000 & -0.000 & -0.001 & -0.001 & -0.002 \\
\hline $\mathcal{F}_{\text {osc }}$ & $\Delta \mathcal{A}$ & -0.007 & +0.006 & +0.002 & -0.003 & -0.005 & -0.007 & -0.010 & -0.017 & -0.028 & -0.036 & -0.039 \\
\hline$\Delta N_{D s}$ & $\Delta \mathcal{A}$ & -0.000 & $\frac{-0.000}{+0.000}$ & $\frac{-0.000}{-0.001}$ & $\frac{-0.000}{+0.000}$ & $\frac{-0.000}{+0.000}$ & $\frac{-0.000}{+0.002}$ & $\frac{-0.000}{+0.002}$ & $\frac{-0.000}{+0.003}$ & $\frac{-0.001}{+0.004}$ & $\frac{-0.001}{+0.005}$ & $\frac{0.001}{+0.001}$ \\
\hline & $\Delta \sigma$ & +0.000 & +0.000 & +0.000 & +0.000 & $\begin{array}{r}+0.001 \\
\end{array}$ & +0.001 & +0.001 & +0.002 & +0.003 & +0.004 & +0.004 \\
\hline$\Delta N_{r e f l}$ & $\Delta \mathcal{A}$ & -0.002 & +0.001 & +0.000 & +0.001 & +0.001 & +0.001 & +0.001 & +0.003 & +0.005 & +0.006 & +0.002 \\
\hline & $\Delta \sigma$ & +0.000 & +0.000 & +0.000 & +0.000 & +0.001 & +0.001 & +0.001 & +0.001 & +0.002 & +0.002 & +0.003 \\
\hline $\operatorname{Br}\left(D_{s} D_{s}\right)=6.8 \%$ & $\Delta \mathcal{A}$ & +0.000 & -0.000 & -0.000 & -0.000 & -0.000 & +0.000 & +0.000 & -0.001 & -0.002 & -0.001 & -0.002 \\
\hline & $\Delta \sigma$ & +0.000 & +0.000 & +0.000 & +0.000 & +0.000 & +0.001 & +0.001 & +0.001 & +0.001 & +0.002 & +0.002 \\
\hline $\operatorname{Br}\left(D_{s} \mu X\right)=5.5 \%$ & $\Delta \mathcal{A}$ & +0.012 & -0.004 & -0.009 & -0.006 & -0.006 & -0.002 & +0.002 & -0.006 & -0.008 & -0.001 & -0.016 \\
\hline & $\Delta \sigma$ & +0.001 & +0.002 & +0.002 & +0.003 & +0.003 & +0.004 & +0.005 & +0.007 & +0.008 & +0.010 & +0.011 \\
\hline$\epsilon$ versus VPDL & $\Delta \mathcal{A}$ & 0.000 & 0.000 & 0.000 & 0.000 & 0.000 & 0.000 & 0.000 & 0.000 & 0.000 & 0.000 & 0.000 \\
\hline & $\Delta \sigma$ & +0.001 & +0.002 & +0.002 & +0.003 & +0.003 & +0.004 & +0.005 & +0.007 & +0.008 & +0.010 & +0.011 \\
\hline$s f_{s i g}$ & $\Delta \mathcal{A}$ & +0.001 & +0.000 & -0.007 & +0.003 & +0.023 & -0.007 & +0.018 & -0.020 & +0.030 & -0.031 & +0.074 \\
\hline & $\Delta \sigma$ & +0.000 & +0.001 & +0.002 & +0.003 & +0.002 & -0.001 & -0.008 & -0.022 & -0.044 & -0.067 & -0.085 \\
\hline$B_{s}$ & $\Delta \mathcal{A}$ & +0.006 & +0.002 & -0.003 & +0.002 & +0.006 & +0.019 & +0.009 & +0.008 & -0.016 & +0.017 & -0.013 \\
\hline dilution & $\Delta \sigma$ & +0.003 & +0.005 & +0.008 & +0.012 & +0.017 & +0.024 & +0.032 & +0.044 & +0.060 & +0.079 & +0.103 \\
\hline$B_{u}$ & $\Delta \mathcal{A}$ & +0.005 & -0.002 & -0.001 & +0.001 & +0.002 & +0.000 & -0.001 & +0.001 & +0.006 & +0.003 & +0.001 \\
\hline dilution & $\Delta \sigma$ & +0.000 & +0.000 & +0.000 & +0.000 & +0.000 & +0.000 & +0.000 & +0.000 & +0.000 & +0.000 & +0.001 \\
\hline Non-zero $\Delta \Gamma / \Gamma$ & $\Delta \mathcal{A}$ & +0.000 & -0.000 & +0.002 & -0.001 & +0.001 & -0.004 & -0.005 & -0.004 & -0.002 & -0.013 & +0.005 \\
\hline & $\Delta \sigma$ & +0.000 & -0.000 & -0.001 & -0.001 & -0.002 & -0.003 & -0.004 & -0.006 & -0.009 & -0.013 & -0.016 \\
\hline Parameter & $\Delta \mathcal{A}$ & +0.000 & +0.000 & +0.000 & +0.000 & +0.000 & +0.001 & +0.002 & +0.003 & +0.002 & +0.007 & +0.004 \\
\hline correlations & $\Delta \sigma$ & -0.000 & -0.000 & +0.000 & +0.000 & +0.000 & +0.000 & +0.001 & +0.001 & +0.002 & +0.003 & +0.004 \\
\hline K-factor variation & $\Delta \mathcal{A}$ & +0.000 & -0.004 & +0.003 & -0.005 & +0.019 & -0.046 & -0.069 & +0.111 & +0.047 & -0.036 & +0.098 \\
\hline $2 \%$ & $\Delta \sigma$ & -0.000 & -0.001 & -0.002 & -0.003 & -0.006 & -0.009 & -0.016 & -0.029 & -0.033 & -0.052 & -0.035 \\
\hline K-factor trigger $\epsilon$ & $\Delta \mathcal{A}$ & +0.000 & +0.000 & +0.000 & -0.002 & -0.002 & +0.002 & -0.003 & -0.004 & +0.013 & +0.024 & -0.001 \\
\hline uncertainty & $\Delta \sigma$ & 0.000 & -0.000 & -0.000 & -0.000 & -0.000 & -0.000 & -0.000 & -0.000 & +0.000 & +0.000 & -0.001 \\
\hline Total syst. & $\sigma_{\text {tot }}^{\text {sys }}$ & 0.083 & 0.073 & 0.075 & 0.075 & 0.087 & 0.098 & 0.107 & 0.111 & 0.069 & 0.098 & 0.112 \\
\hline Total & $\sigma_{t o t}$ & 0.091 & 0.106 & 0.138 & 0.184 & 0.257 & 0.358 & 0.489 & 0.662 & 0.877 & 1.144 & 1.421 \\
\hline
\end{tabular}


Table 4.4. Systematic uncertainties on the amplitude for combined RunIIa and RunIIb. The shifts of both the measured amplitude, $\Delta \mathcal{A}$, and its statistical uncertainty, $\Delta \sigma$, are listed.

\begin{tabular}{|c|c|c|c|c|c|c|c|c|c|c|c|}
\hline Osc. frequency & & $0 p s^{-1}$ & $3 p s^{-1}$ & $6 p s^{-1}$ & $9 p s^{-1}$ & $12 \mathrm{ps}^{-1}$ & $15 \mathrm{ps}^{-1}$ & $18 p s^{-1}$ & $21 p s^{-1}$ & $24 p s^{-1}$ & $27 p s^{-1}$ \\
\hline $\mathcal{A}$ & & 0.0046 & -0.0033 & 0.0038 & -0.0391 & -0.2155 & 0.5176 & 0.8695 & 0.2489 & -0.2504 & 0.2648 \\
\hline Stat. Uncertainty & & 0.0352 & 0.0585 & 0.0904 & 0.1325 & 0.1924 & 0.2725 & 0.3795 & 0.5253 & 0.6845 & 0.9057 \\
\hline \multirow[t]{2}{*}{$\mathrm{c} \tau_{B s}$} & $\Delta \mathcal{A}$ & -0.0002 & -0.0000 & -0.0000 & -0.0002 & -0.0005 & 0.0020 & 0.0028 & 0.0001 & -0.0017 & 0.0007 \\
\hline & $\Delta \sigma_{\mathcal{A}}$ & 0.0000 & 0.0001 & 0.0001 & 0.0002 & 0.0004 & 0.0006 & 0.0009 & 0.0014 & 0.0019 & 0.0028 \\
\hline \multirow[t]{2}{*}{$\mathrm{c} \tau_{B s}=439 \mu \mathrm{m}$} & $\Delta \mathcal{A}$ & 0.0011 & 0.0002 & -0.0005 & 0.0009 & 0.0019 & -0.0082 & -0.0118 & 0.0003 & 0.0083 & -0.0000 \\
\hline & & -0.0001 & -0.0002 & -0.0005 & -0.0009 & -0.0015 & -0.0023 & -0.0035 & -0.0053 & -0.0074 & \\
\hline \multirow[t]{2}{*}{$\mathcal{F}_{\text {peak_sig }}$} & $\Delta \mathcal{A}$ & -0.0001 & 0.0001 & -0.0003 & -0.0007 & -0.0035 & 0.0030 & 0.0061 & -0.0052 & -0.0172 & $\begin{array}{r}-0.0111 \\
0.0118\end{array}$ \\
\hline & $\Delta \sigma_{\mathcal{A}}$ & $\frac{0.0001}{0.0004}$ & $\frac{0.0002}{0.0001}$ & 0.0003 & 0.0006 & $\frac{0.0011}{-0.0002}$ & $\frac{0.0018}{0.0009}$ & $\frac{0.0031}{0.0018}$ & $\frac{0.0052}{0.0010}$ & $\frac{0.0077}{0.0002}$ & $\frac{0.0118}{0.0021}$ \\
\hline $\mathcal{F}_{\text {peak_bkg }}$ & $\begin{array}{l}\Delta \mathcal{A} \\
\Delta \sigma_{A}\end{array}$ & $\begin{array}{r}0.0004 \\
-0.0000\end{array}$ & $\begin{array}{r}0.0001 \\
-0.0000\end{array}$ & $\begin{array}{l}0.0000 \\
0.0000\end{array}$ & $\begin{array}{l}0.0000 \\
0.0000\end{array}$ & $\begin{array}{r}-0.0002 \\
0.0001\end{array}$ & $\begin{array}{l}0.0009 \\
0.0002\end{array}$ & $\begin{array}{l}0.0003 \\
0.003\end{array}$ & $\begin{array}{l}0.0010 \\
0.0005\end{array}$ & 0.0008 & 0.0012 \\
\hline \multirow{2}{*}{$\sigma_{\text {peak_bkg }}$} & $\Delta \mathcal{A}$ & 0.0000 & -0.0000 & -0.0001 & 0.0001 & 0.0003 & -0.0001 & -0.0006 & 0.0000 & 0.0007 & -0.0007 \\
\hline & $\overline{\Delta \sigma_{\mathcal{A}}}$ & 0.0000 & -0.0000 & 0.0000 & -0.0000 & -0.0000 & -0.0001 & -0.0001 & -0.0003 & -0.0004 & -0.0007 \\
\hline \multirow[t]{2}{*}{$\mathcal{F}^{0}$} & $\Delta \mathcal{A}$ & 0.0005 & 0.0001 & 0.0001 & $\overline{0.0}$ & -0.1 & 0.0018 & 0.0037 & 0.0023 & 0.0007 & 0.0054 \\
\hline & $\overline{\Delta \sigma_{\mathcal{A}}}$ & -0.0000 & -0.0000 & 0.0000 & 0.0 & & 0.0003 & 0.0006 & 0.0011 & 0.0017 & 0.0027 \\
\hline \multirow{2}{*}{$\mathrm{c} \tau_{b k g}$} & $\Delta \mathscr{A}$ & 0.0002 & -0.0000 & 0.0000 & 0.0000 & 0.0001 & -0.0004 & -0.0004 & -0.0001 & -0.0000 & -0.0001 \\
\hline & $\Delta \sigma_{\mathcal{A}}$ & -0.0000 & -0.0000 & -0.0000 & -0.0001 & -0.0001 & -0.0001 & -0.0002 & -0.0002 & -0.0003 & -0.0004 \\
\hline \multirow{2}{*}{$s f_{b k g}$} & $\Delta \mathcal{A}$ & 0.0001 & -0.0001 & -0.0002 & -0.0004 & -0.0004 & -0.0007 & -0.0009 & -0.0008 & -0.0007 & -0.0010 \\
\hline & $\Delta \sigma_{\mathcal{A}}$ & -0.0000 & -0.0000 & -0.0000 & -0.0000 & -0.0000 & -0.0000 & -0.0000 & -0.0001 & -0.0001 & -0.0001 \\
\hline \multirow[t]{2}{*}{$\mathcal{F}_{\text {tsens }}$} & $\Delta \mathcal{A}$ & -0. & & 0. & & & -0.0 & & & 91 & -0.0225 \\
\hline & $\Delta \sigma_{\mathcal{A}}$ & -0.0000 & -0.0000 & -0.0001 & -0.0000 & -0.0001 & -0.0001 & -0.0002 & -0.0001 & -0.0003 & -0.0005 \\
\hline \multirow[t]{2}{*}{$\mathcal{F}_{\text {osc }}$} & $\Delta \mathcal{A}$ & -0.0195 & 0 & 0.0 & -0 . & -0 & -0. & -0 & & 29 & -0.0310 \\
\hline & $\Delta \sigma_{\mathcal{A}}$ & -0.0000 & -0.0 & -0.0 & -0. & & -0.0000 & -0.0 & 000 & -0.0002 & -0.0003 \\
\hline \multirow[t]{2}{*}{$\Delta N_{D s}$} & $\Delta \mathcal{A}$ & -0.0010 & 0.0002 & 0.0 & -0.0 & -0. & 0.0 & 0. & 0.0021 & $\pi 16$ & 0.0023 \\
\hline & $\Delta \sigma_{\mathcal{A}}$ & & & 0 & & & 08 & & & 0.0021 & 0.0028 \\
\hline \multirow{2}{*}{$\Delta N_{\text {refl }}$} & $\Delta \mathcal{A}$ & -0.0 & 0.0 & 0.0 & 0. & & 02 & 28 & 43 & 0.0057 & 0.0048 \\
\hline & $\Delta \sigma_{\mathcal{A}}$ & 0.0001 & 0.0001 & 02 & 03 & 0. & $0 .($ & 08 & 0.0012 & 0.0016 & 0.0020 \\
\hline \multirow{2}{*}{$B_{s}^{0} \rightarrow D_{s} D_{s}=6.8 \%$} & $\Delta \mathcal{A}$ & -0.0001 & -0.0000 & -0.0 & -0. & -0. & 0. & 16 & 012 & -0.0025 & -0.0014 \\
\hline & $\Delta \sigma_{\mathcal{A}}$ & 0.0 & 0.0 & 0 & & & 05 & 07 & b09 & 0.0011 & 0.0015 \\
\hline \multirow[t]{2}{*}{$B_{s}^{0} \rightarrow D_{s} \mu \nu X=5.5 \%$} & $\Delta \mathcal{A}$ & 0.0124 & -0.0 & -0.0 & -0. & -0 . & 0.0 & 13 & -0.0031 & -0.0070 & -0.0035 \\
\hline & $\Delta \sigma_{\mathcal{A}}$ & & & & & & 0.0029 & 0.0039 & 50 & 0.0062 & 0.0080 \\
\hline \multirow[t]{2}{*}{$\epsilon$ versus VPDL } & $\Delta \mathcal{A}$ & 0.0 & & 0. & & 00 & 0.0000 & & 0.0000 & 0.0000 & 0.0000 \\
\hline & $\overline{\Delta \sigma_{\mathcal{A}}}$ & & & & & & & & & & 907 \\
\hline \multirow[t]{2}{*}{$s f_{s i g}$} & $\Delta \mathcal{A}$ & 0.0001 & 0.0017 & -0.0027 & 0.0052 & 0.0186 & -0.0052 & 0.0158 & 0.0104 & -0.0376 & -0.1733 \\
\hline & $\Delta \sigma_{\mathcal{A}}$ & 0.0001 & 0.0008 & 0.0 & & & $-0 .($ & $-0 .($ & & 22 & -0.0505 \\
\hline \multirow[t]{2}{*}{$B_{s}^{0}$ dilution } & $\Delta \mathcal{A}$ & 0.0038 & -0.0026 & 0.0008 & $\overline{-0.0}$ & -0.0 & 0.0 & 0.0471 & 0.0119 & -0.0338 & -0.0059 \\
\hline & $\overline{\Delta \sigma_{\mathcal{A}}}$ & & & & & & & & & & 0.0597 \\
\hline \multirow[t]{2}{*}{$B_{u}$ dilution } & $\Delta \mathcal{A}$ & 0.0073 & -0.0020 & -0.0011 & -0.0004 & 07 & 0.0 & -0.0003 & 0.0016 & 0.0046 & 0.0051 \\
\hline & $\Delta \sigma_{\mathcal{A}}$ & & & & & & & & & & 002 \\
\hline \multirow[t]{2}{*}{ Non-zero $\Delta \Gamma / \Gamma$} & $\Delta \mathcal{A}$ & 0.0011 & 0.0002 & -0.0004 & 0.0009 & 0.0019 & -0.0086 & -0.0127 & 0.0003 & 0.0082 & -0.0002 \\
\hline & $\Delta \sigma_{\mathcal{A}}$ & & & & & & & & & -0.0078 & -0.0113 \\
\hline correlations & $\Delta \mathcal{A}$ & 0.0001 & -0.0000 & 0.0001 & 0.0 & 0.0 & 0.0008 & 0.0019 & 0.0013 & 0.0006 & 0.0033 \\
\hline & $\overline{\Delta \sigma_{\mathcal{A}}}$ & -0.0 & & & & & & 0.0003 & 0.0005 & 0.0009 & 0.0015 \\
\hline K-factor variation $2 \%$ & $\Delta \mathcal{A}$ & 0.0009 & -0.0033 & -0.0026 & 112 & -0.0148 & -0.0584 & -0.0724 & 0.1710 & -0.0004 & 0.0546 \\
\hline & $\Delta \sigma_{\mathcal{A}}$ & -0.0 & & & & & & -0.0132 & -0.0185 & -0.0237 & -0.0420 \\
\hline K-factor trigger $\epsilon$ & $\Delta \mathcal{A}$ & 0.0000 & & & & & -0.0 & 0.0 & & -0.0038 & 0.0181 \\
\hline & $\overline{\Delta \sigma_{\mathcal{A}}}$ & -0.0000 & -0.0000 & -0.0000 & -0.0000 & -0.0000 & -0.0000 & -0.0001 & 0.0000 & 0.0001 & 0.0006 \\
\hline Total syst. & $\sigma_{t o t}^{\text {sys }}$ & 0.0876 & 0.0714 & 0.0746 & 0.0776 & 0.0932 & 0.1021 & 0.1209 & 0.1617 & 0.1229 & 0.2249 \\
\hline Total & totot & 0.0944 & 0.0924 & 0.1172 & 0.1536 & 0.2138 & 0.2910 & 0.3983 & 0.5497 & 0.6954 & 0.9332 \\
\hline
\end{tabular}




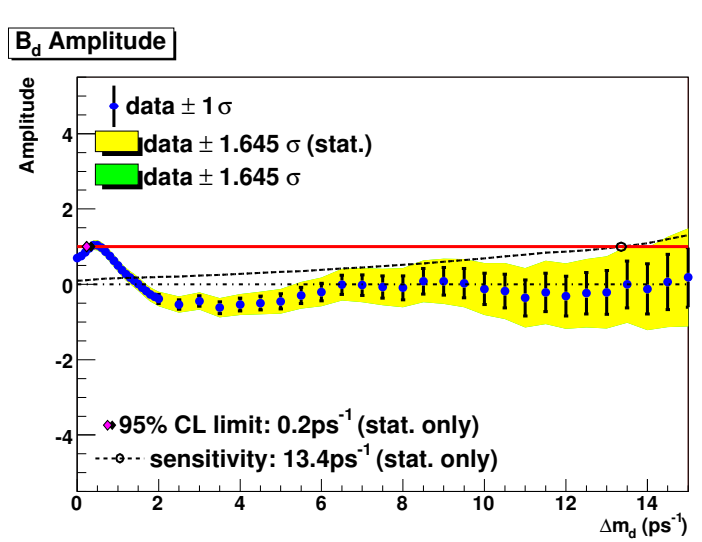

Figure 4.12. $B_{d}-\bar{B}_{d}$ oscillation amplitude in RunIIa data.

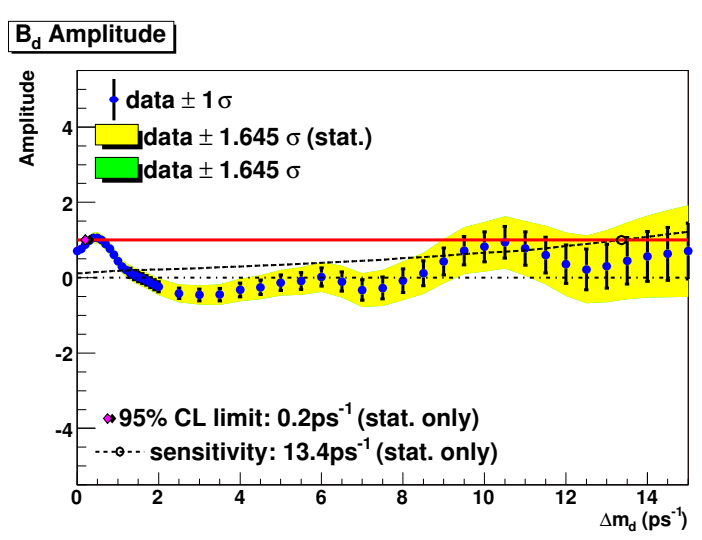

Figure 4.14. $B_{d}-\bar{B}_{d}$ oscillation amplitude in RunIIb data.

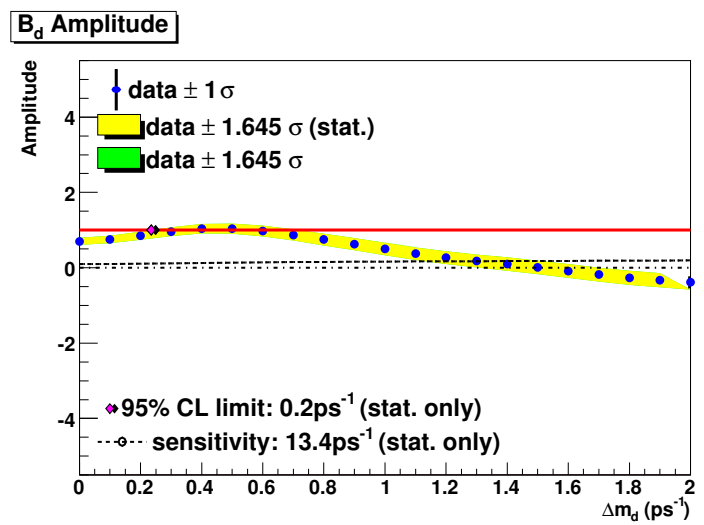

Figure 4.13. $B_{d}-\bar{B}_{d}$ oscillation amplitude (detailed view of the $B_{d}$ oscillation region) in RunIIa data.

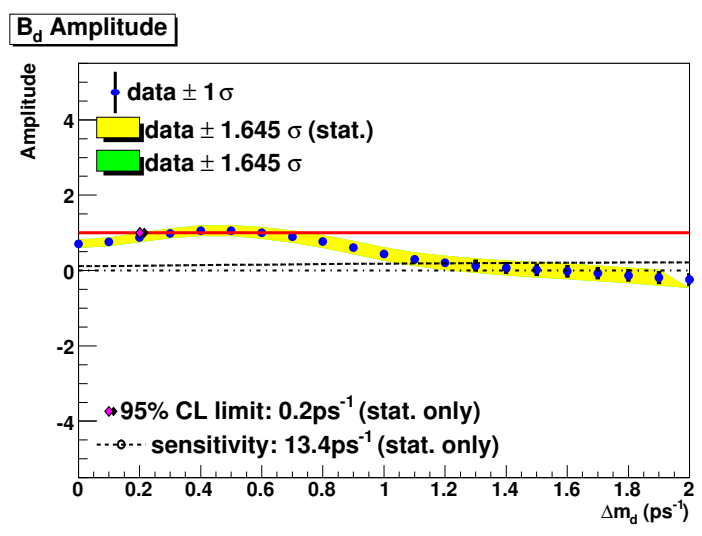

Figure 4.15. $\quad B_{d}-\bar{B}_{d}$ oscillation amplitude (detailed view of the $B_{d}$ oscillation region) in RunIIb data.

\subsubsection{Events Biased by Impact Parameter Triggers}

There are a number of triggers used at D-Zero that have impact parameter requirements.

Because this could cause a bias in the lifetime measurement, the events flagged as IP biased were studied. A biased event is defined as an event that was triggered only by one or more impact-parameter triggers. A complete list of impact parameter triggers for 
RunIIa and RunIIb was obtained with the assistance of Rick Jessik and Kin Yip and the lists are shown in Appendix $\mathrm{C}$ An event triggered only by IP triggers is flagged as biased for later study.

Figure 4.16] shows the mass plot for the IP biased events in RunIIa, with the same shown in Figure 4.17 for RunIIb. We fit the $B_{s}^{0}$ lifetime in each sample. In RunIIa a value of $c \tau_{B_{s}}=(581.931 \pm 11.908) \mu \mathrm{m}$ was obtained, and in RunIIb the value is $c \tau_{B_{s}}=$ $(533.024 \pm 10.1516) \mu \mathrm{m}$; these values verify the assumption that IP-biased events bias the measured $B_{s}^{0}$ lifetime towards higher values.

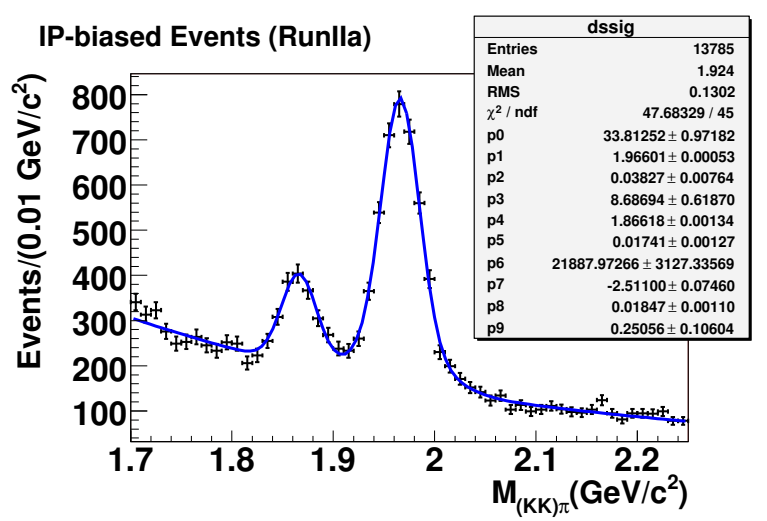

Figure 4.16 .

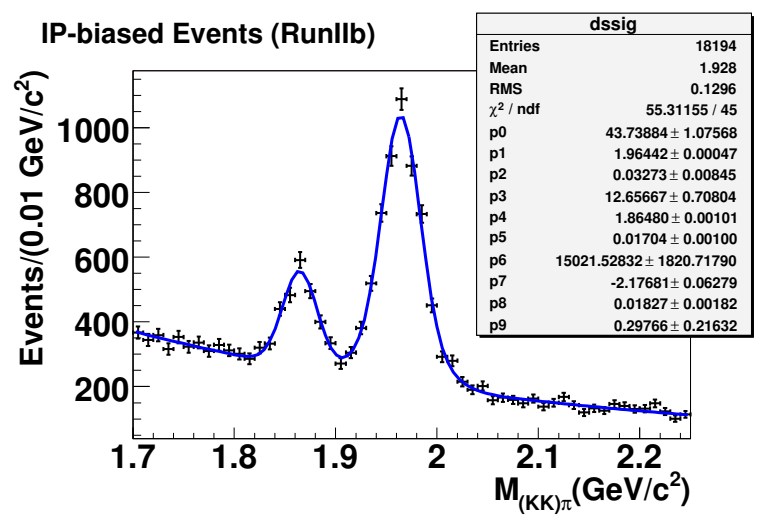

Figure 4.17.

As mentioned previously, the measured lifetime does not impact the result for $\Delta m_{s}$. To support this argument, the IP-biased events were excluded from the sample selection and new $p d f s$ and mass fit parameters were inputted for the $\Delta m_{s}$ fit. Amplitude scans were performed, and the results are shown in Figures 4.18 and 4.19, Comparing these plots to the results plots in Figures 4.1 and 4.2, shows that they have identical shapes; the main difference is that the inclusion of IP-biased events leads to a higher sensitivity to $\Delta m_{s}$. 


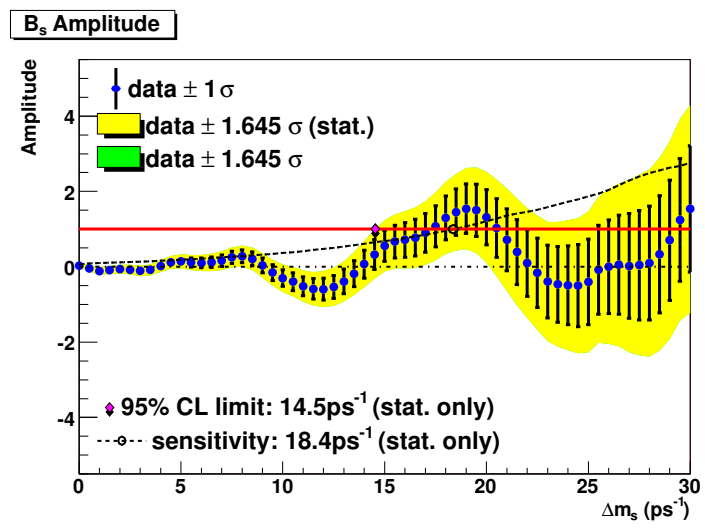

Figure 4.18 .

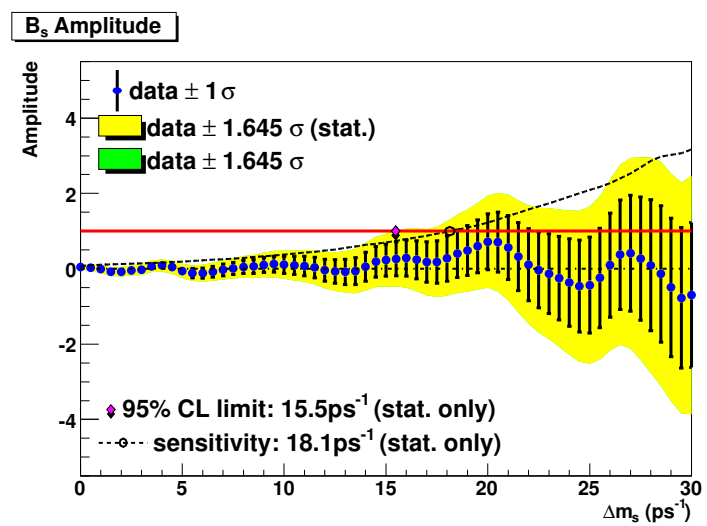

Figure 4.19.

\subsection{Determination of the Significance of the Result}

In order to determine the quality of the result obtained, the significance must be determined. The determination of the statistical significance is discussed first, with a discussion of the significance of the log-likelihood scan following. Additionally, if a measurement of the frequency $\Delta m_{s}$ is possible, then the statistical and systematic uncertainties on the measured value must be obtained; this discussion is given last.

\subsubsection{Randomized Flavor Tagging}

To determine the statistical significance of the result, a cross-check was performed with the flavor tagging randomized. This is done by randomizing the sign of the dilution and fitting for $\Delta m_{s}$. The dilution for the $B_{d}$ components was kept properly flavor-tagged; this gives the correct description of an infinite $\Delta m_{s}$, which this study effectively emulates. The result of this fit for RunIIa data is shown in Figure 4.20 and RunIIb data is shown in Figure 4.21. 


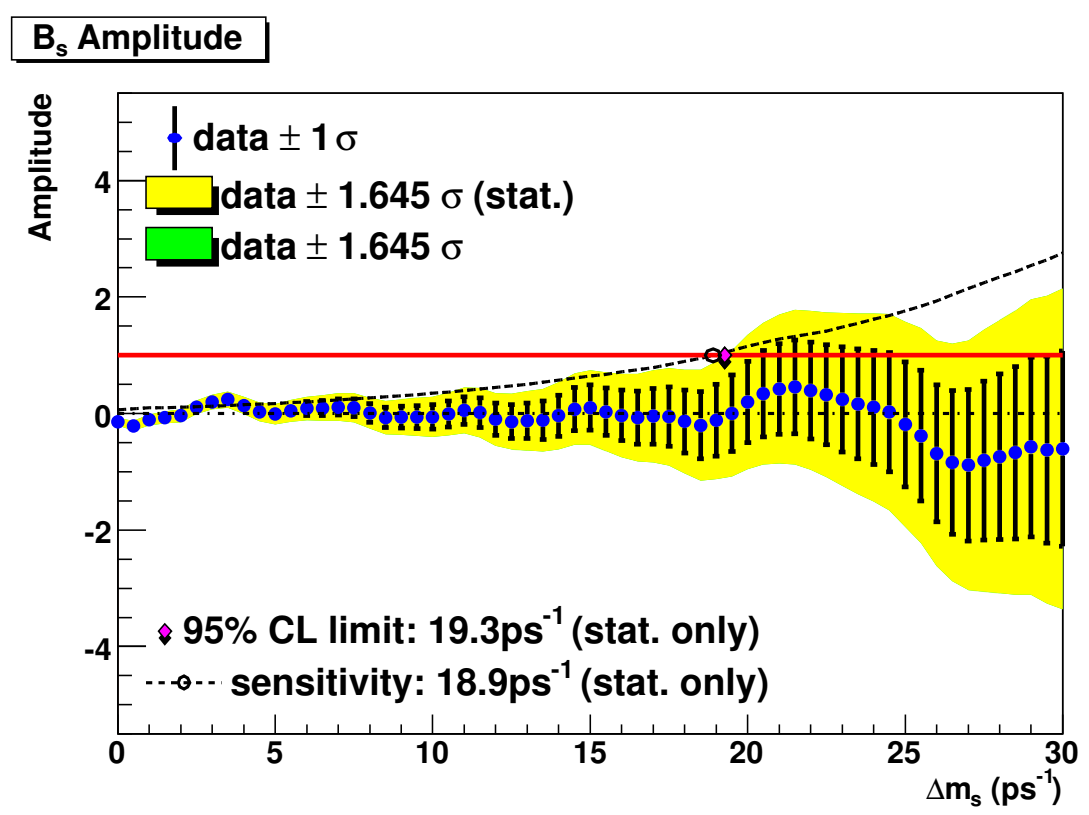

Figure 4.20. $\quad B_{s}^{0}$ oscillation amplitude with statistical errors only for RunIIa data, with flavor tagging randomized.

The "experiment" of flavor-tagging randomization was performed 1000 times on both the RunIIa and RunIIb dataset, each time using a different seed for the random-number generator and fitting for the amplitude at $\Delta m_{s}=19 \mathrm{ps}^{-1}$. For each amplitude corresponding to a different seed, the amplitude and its uncertainty at each $\Delta m_{s}$ value is saved. For each seed, both the individual results for RunIIa and RunIIb, as well as a weighted average of the amplitudes and their uncertainties from RunIIa and RunIIb is taken. These values are then plotted in a distribution. These distributions are shown in Figure 4.22. The statistical significance is determined by the number of "experiments" that meet or exceed the result obtained. In RunIIa, 4 experiments had amplitudes greater than the result amplitude; this corresponds to a greater than $2.5 \sigma$ significance. In RunIIb, 167 experiments had amplitudes greater than the result amplitude, which indicates a less 


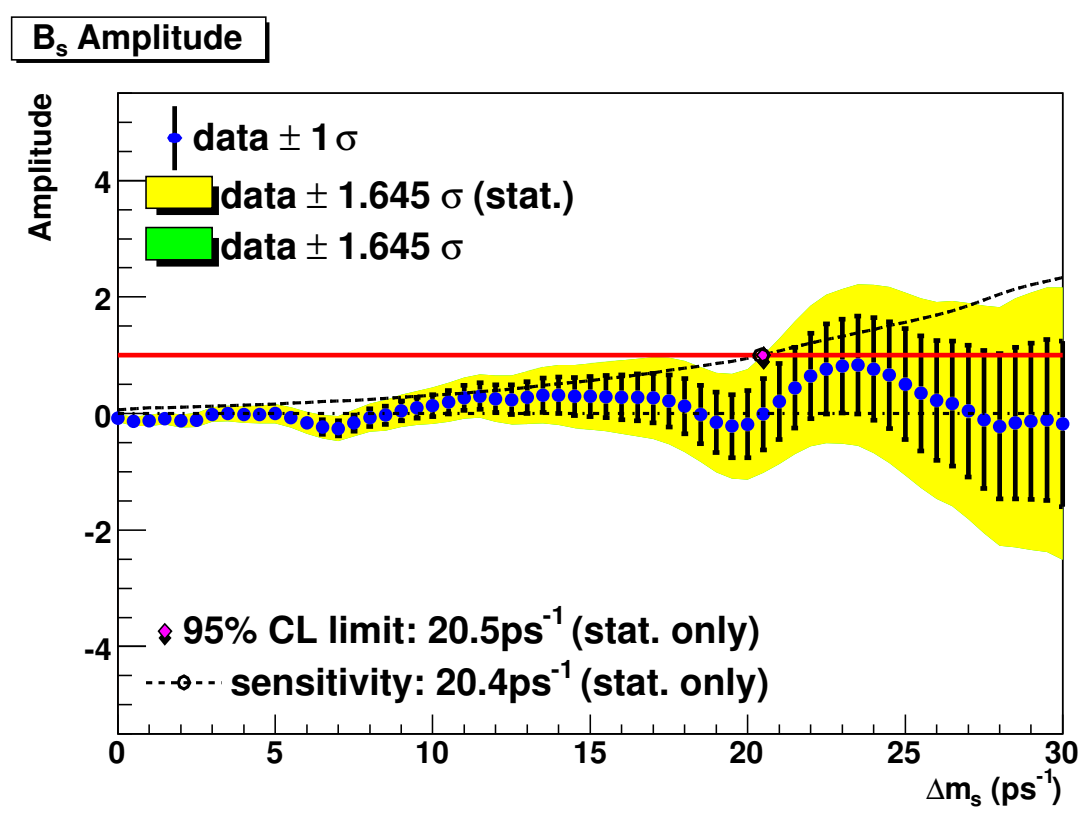

Figure 4.21. $\quad B_{s}^{0}$ oscillation amplitude with statistical errors only for RunIIb data, with flavor tagging randomized.

than $1 \sigma$ significance. The combined RunIIa+RunIIb experiments indicated a $2.6 \sigma$ significance, with 10 out of the 1000 experiments having amplitudes greater than the result.

\subsubsection{Log-Likelihood Determination of $\Delta m_{s}$}

The log-likelihood scan of $\Delta m_{s}$ shown in Figure 4.7 includes statistical uncertainties only. Another log-likelihood curve is obtained for the statistical and systematic uncertainties using the final combined amplitude scan of RunIIa and RunIIb. These two curves are plotted together in Figure 4.23. The curve including both statistical and systematic uncertainties shows a broader parabolic curve. The minima of each curve gives a value for $\Delta m_{s}$ of $18.86 \mathrm{ps}^{-1}$. By taking the difference in widths of the two log-likelihood curves, an estimation of the systematic uncertainty on $\Delta m_{s}$ is obtained. The difference 
in widths leads to a systematic uncertainty of $0.37 \mathrm{ps}^{-1}$. This gives a final result for $\Delta m_{s}$ of $\Delta m_{s}=18.86 \pm 0.80($ stat $) \pm 0.37($ sys $) \mathrm{ps}^{-1}$. In addition, the statistical significance calculated from the flavor-tagging randomization above can be compared to the statistical significance of the log-likelihood scan. The difference between the log-likelihood at the minimum and the log-likelihood at infinite $\Delta m_{s}$ gives an estimate of the significance of the result. Reference [70] shows a method for calculating the significance from the amplitude and amplitude uncertainty at the result frequency. Using this method, we obtain a significance of 2.3 , which is in agreement with the significance calculated in the previous section. 

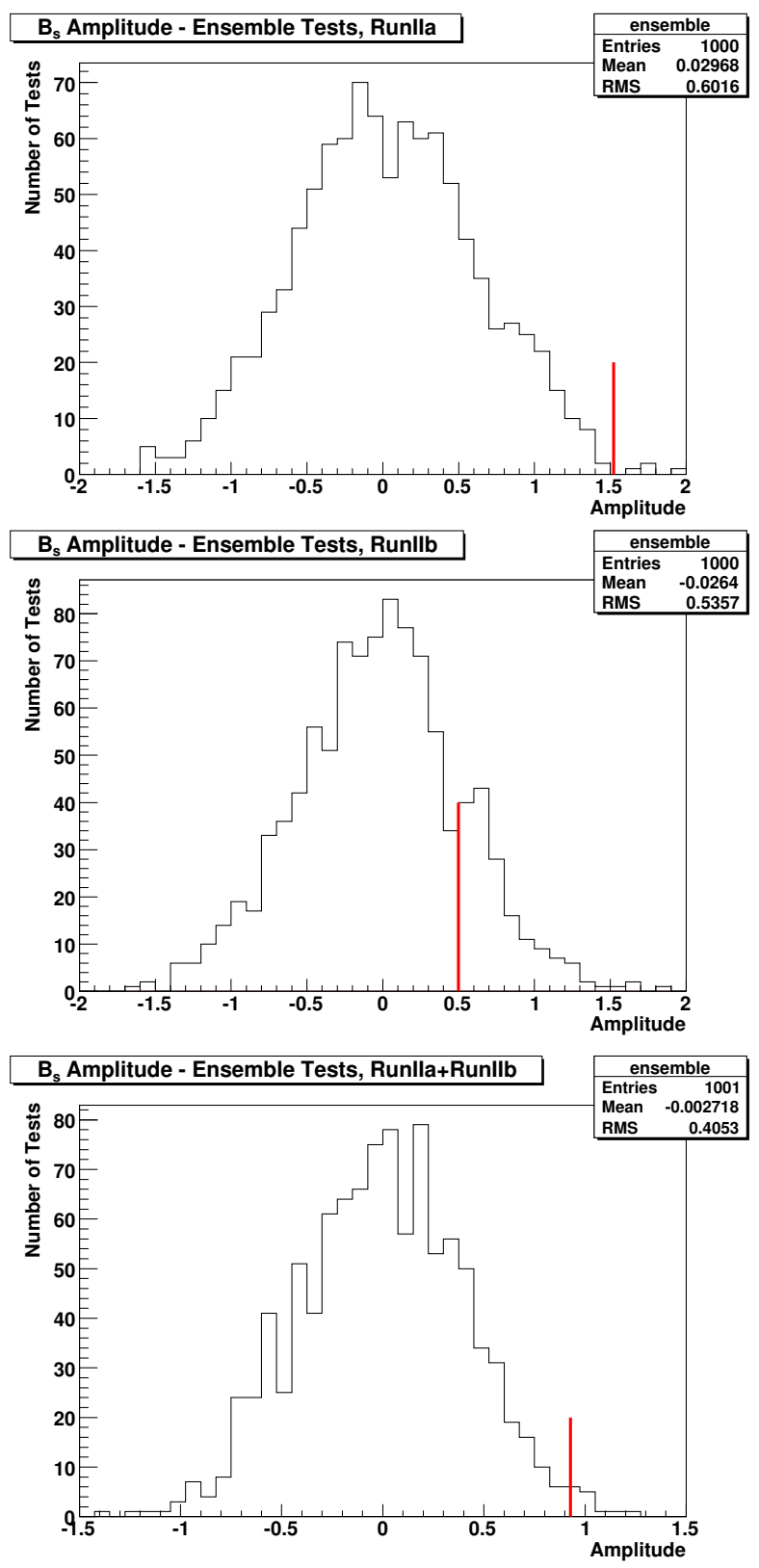

Figure 4.22. Amplitude results from the flavor-tagging randomization experiments. The red vertical lines indicates the flavor-tagged result amplitude at $\Delta m_{s}=19 \mathrm{ps}^{-1}$; experiment results to the right of this line indicate an amplitude greater than the data amplitude. RunIla is shown in the upper-most plot, RunIIb in the center, and the combined RunIIa+RunIIb results are shown in the lower plot. 


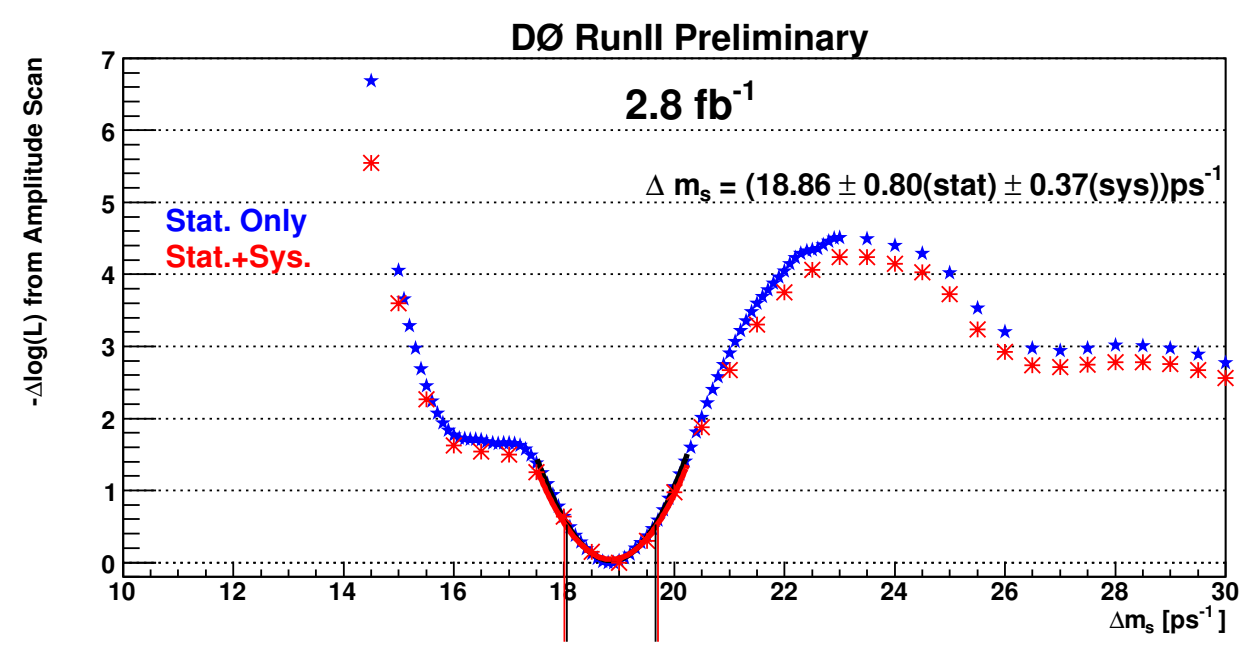

Figure 4.23. Log-likelihood curve with statistical and systematic uncertainties included. The blue points are the log-likellihood curve with only statistical uncertainties included, while the red points are for statistical and systematic uncertainties. A polynomial is fitted to each curve, and the difference in widths indicates the systematic uncertainty on $\Delta m_{s}$. 


\subsection{Conclusions}

Using a signal of 27,800 RunIIa $B_{s}^{0} \rightarrow \mu^{+} \nu D_{s}^{-} X$ decays where $D_{s}^{-} \rightarrow \phi \pi^{-}, \phi \rightarrow K^{+} K^{-}$ and a combined opposite-side and same-side flavor tagging algorithm we performed a search for $B_{s}^{0}-\bar{B}_{s}^{0}$ oscillations. We obtained a $95 \%$ confidence level limit on the oscillation frequency $\Delta m_{s}>14.4 \mathrm{ps}^{-1}$ and a sensitivity of $18.7 \mathrm{ps}^{-1}$, with statistical and systematic errors.

A signal of 16,000 RunIIb $B_{s}^{0} \rightarrow \mu^{+} \nu D_{s}^{-} X$ decays where $D_{s}^{-} \rightarrow \phi \pi^{-}, \phi \rightarrow K^{+} K^{-}$ and a combined opposite-side and same-side flavor tagging algorithm we obtained a 95\% confidence level limit on the oscillation frequency $\Delta m_{s}>16.0 \mathrm{ps}^{-1}$ and a sensitivity of $20.3 \mathrm{ps}^{-1}$, with statistical and systematic errors.

We obtained a combined result for RunIIa and RunIIb data, with a 95\% confidence level limit on the oscillation frequency $\Delta m_{s}>23.2 \mathrm{ps}^{-1}$ and a sensitivity of $23.0 \mathrm{ps}^{-1}$, with statistical and systematic errors included. We find a minimum log-likelihood value of $\Delta m_{s}=18.86 \mathrm{ps}^{-1}$. The statistical significance of the result is $2.6 \sigma$, and the final measurement of the $B_{s}^{0}$ oscillation frequency is $\Delta m_{s}=18.86 \pm 0.80($ stat $) \pm 0.37($ sys $)$.

\subsection{Discussion}

The result obtained in this thesis is the most-precise single-decay result ever obtained at D-Zero. The value of $\Delta m_{s}$ obtained agrees with previous measurements from DZero [17] and CDF [10, 46].

The measurement of $\Delta m_{s}$ obtained in this analysis can be used to determine a constraint on the CKM matrix element $\left|V_{t d}\right|$. This can be done by rearranging Equation 1.28 
and inputting the other theoretical and experimental values. This gives:

$$
\frac{\left|V_{t d}\right|}{\left|V_{t s}\right|}=\xi \sqrt{\frac{\Delta m_{d} m_{B s}}{\Delta m_{s} m_{B d}}},
$$

where $\xi=1.210_{-0.035}^{+0.047}$ as mentioned in Chapter 1, $m_{B s}=5366.4 \pm 1.1 \mathrm{MeV}, m_{B d}=$ $5279.5 \pm 0.5 \mathrm{MeV}$, and $\Delta m_{d}=0.507 \pm 0.005 \mathrm{ps}^{-1}[\mathbf{6}$. Using our result obtained here for $\Delta m_{s}$, we find:

$$
\frac{\left|V_{t d}\right|}{\left|V_{t s}\right|}=0.200008 \pm 0.0047(\text { exp. })_{-0.0058}^{+0.0078}(\text { theo. })
$$

This agrees with the value from HFAG given in Equation 1.29, and shows that the uncertainty on this ratio of CKM elements is dominated by the theoretical uncertainty. Better constraints on the CKM matrix elements will become available with future improvements in the uncertainty on the theoretical component, $\xi$. 


\section{References}

[1] M. Kobayashi and T. Maskawa, Progr. Theor. Phys. 49, 652 (1973).

[2] This follows the discussion of the Standard Model in: D. Griffiths, Introduction to Elementary Particles, New York: John Wiley \& Sons, INC (1987).

[3] N. Cabibbo, Phys. Rev. Lett. 10, 531 (1963).

[4] S. L. Glashow, J. Iliopoulos, and L. Maiani, Phys. Rev. D 2, 1285 (1970).

[5] L. Wolfenstein, Phys. Rev. Lett. 51, 1945 (1983).

[6] W.-M. Yao et al., Particle Data Group (PDG), J. Phys. G 33, 1 (2006).

[7] R. Waldi, "Flavour Oscillation and CP Violation of B Mesons", Prog. Part. Nucl. Phys. 47, 1-71 (2001); K. Anikeev et al., "B Physics at the Tevatron: RunII and Beyond", (2002) arXiv:hep-ph/0201071v2.

[8] CKMfitter Group, http://ckmfitter.in2p3.fr/

[9] Heavy Flavor Averaging Group (HFAG), Summer 2007 averages, http://www.slac.stanford.edu/xorg/hfag/semi/LP07/home.shtml and references therein.

[10] A. Abulencia et al. (CDF Collaboration), Phys. Rev. Lett. 97, 242003 (2006).

[11] C. Gay, "B Mixing", Ann. Rev. Nucl. Part. Sct. 50 577-641 (2000); arXiv:hepex/0103016 v1.

[12] M. Okamoto, plenary talk at the XXIIIrd International Symposium on Lattice Field Theory, Dublin, July 2005, hep-lat/0510113; http://www.maths.tcd.ie/lat05/plenary-talks/okamoto.pdf

[13] A. Gray et al. (HPQCD Collaboration), Phys. Rev. Lett. 95, 212001 (2005). 
[14] S. Aoki et al. (JLQCD Collaboration), Phys. Ref. Lett. 91, 212001 (2003).

[15] A. J. Buras, M. Jamin and P. H. Weisz, Nucl. Phys. B 347 (1990) 491.

[16] T. Inami and C.S. Lim, Prog. Theor. Phys. 65, 297 (1981).

[17] V. M. Abazov, et al., Phys. Rev. Lett. 97, 021802 (2006).

[18] "Update on $B_{s}^{0}$ mixing in $B_{s}^{0} \rightarrow D_{s}^{-} e^{+} \nu X, D_{s}^{-} \rightarrow \phi \pi^{-}$decay mode", T. Moulik, D-Zero Note 5435 (2007).

[19] "Bs Mixing Studies with $B_{s}^{0} \rightarrow D_{s}^{-} \mu^{+} \nu X\left(D_{s}^{-} \rightarrow K^{*} K\right)$ Decay using Unbinned Fit and Same-Side Tagging", S. Beale, Md. Naimuddin, W. Taylor, D-Zero Note 5446 (2007).

[20] "A Search for Bs Oscillation Mixing Using $B_{s}^{0} \rightarrow D_{s}^{-} \mu^{+} \nu X\left(D_{s}^{-} \rightarrow K_{S} K\right)$ Decays", G. Borissov, S. Burdin, B.C.K. Casey, D. Krop, R. Van Kooten, D-Zero Conference Note 5254 (2006).

[21] "Reconstruction of a $B_{s}^{0} \rightarrow D_{s}^{-}(\phi \pi) \pi$ signal at D-Zero", T. Kuhl, S. Tapprogge, G. Weber, D-Zero Note 5429 (2007).

[22] "Measurement of Flavor Oscillation Frequency of $B_{s}^{0}$ Mesons at D-Zero", M.S. Anzelc et al., D-Zero Conference Note 5474 (2007).

[23] V. M. Abazov etal., Nucl. Instr. and Methods A 565, 463 (2006).

[24] http://www-d0online.fnal.gov/groups/stt/stt_shift_tutorial.pdf

[25] http://www-d0online.fnal.gov/www/groups/cft/CTT/online/ Overview/overview.html

[26] A. Juste, "Silicon Design", http://d0server1.fnal.gov/projects/ Silicon/www/SMT_files/docs/smt_description.htm

[27] DØ RunIIb Upgrade, Technical Design Report, Silicon Detector. http://d0server1.fnal.gov/projects/run2b/Silicon/www/smt2b/Documentation/ D0_Run2b_TDR_Silicon_Sept24_02.pdf

[28] DØ RunIIb Upgrade, Technical Design Report, Silicon Detector. http://d0server1.fnal.gov/projects/run2b/Silicon/www/smt2b/Documentation/ DO_Run2b_TDR_Silicon_Sept24_02.pdf 
[29] The D-ZeroUpgrate Central Fiber Tracker, Technical Design Report, http://d0server1.fnal.gov/users/stephan/www/CFT_TDR/CFT_TDR.ps

[30] DØ Central Fiber Tracker Group, http://d0server1.fnal.gov/projects/ SciFi/pictures/Cylinder_and_Fiber_assembly_Feb99.html

[31] Design Report of the Central PreShower for the D-ZeroUpgrade, M. Adams et al., http://d0server1.fnal.gov/users/qianj/CPS/doc/dn3104.pdf

[32] Calorimeter Operations Group, http://www-d0online.fnal.gov/www/ groups/cal/cal_main.html

[33] V. M. Abazov et al., Nucl. Instr. and Methods A 552, 372 (2005).

[34] http://www-d0.fnal.gov/RunIIaOperations/TriggerBoard/

[35] http://www-d0online.fnal.gov/www/groups/tm/tm_main.html

[36] http://www-d0.fnal.gov/detector/trigger.html

[37] M. Gell-Mann and A. Pais, Phys. Rev. 97, 1387 (1955).

[38] J.H. Christenson et al., Phys. Rev. Lett. 13, 138 (1964).

[39] C. Albajar et al. (UA1 Collaboration), Phys. Lett. B186, 247 (1987).

[40] H. Albrecht et al. (ARGUS Collaboration), Phys. Lett. B192, 245 (1989).

[41] M. Artuso et al. (CLEO Collaboration), Phys. Rev. Lett. 62, 2233 (1989).

[42] Solar-neutrino Experiments:

- B. T. Cleveland et al., Astrophys. J. 496, 505 (1998).

- J. N. Abdurashitov et al., Phys. Rev. C 60, 055801 (1999).

- W. Hampel et al., Phys. Lett. B 447, 127 (1999).

- S. Fukuda et al., Phys. Lett. B 539, 179 (2002).

- Q. R. Ahmad et al., Phys. Rev. Lett. 87, 071301 (2001); Q. R. Ahmad et al., Phys. Rev. Lett. 89, 011301 (2002); S. N. Ahmed et al., Phys. Rev. Lett. 92, 181301 (2004).

Reactor-antineutrino Experiments:

- K. Eguchi et al., Phys. Rev. Lett. 90, 021802 (2003); T. Araki et al., Phys. Rev. Lett. 94, 081801 (2005).

Atmospheric-neutrino Experiments: 
- K. S. Hirata et al., Phys. Lett. B 280, 146 (1992); Y. Fukuda et al., Phys. Lett. B 335, 237 (1994).

- Y. Fukuda et al., Phys. Rev. Lett. 81, 1562 (1998).

- W. W. M. Allison et al., Phys. Lett. B 449, 137 (1999).

- M. Ambrosio et al., Phys. Lett. B 517, 59 (2001).

Long-baseline Accelerator-neutrino Experiments:

- M. H. Ahn et al., Phys. Rev. Lett. 90, 041801 (2003).

- D. G. Michael et al., Phys. Rev. Lett. 97, 191801 (2006).

[43] A. J. Schwartz, "Measurements of D0-D0bar Mixing and Searches for CP Violation: HFAG Combination of all Data", arXiv:hep-ex/0803.0082 v2 (2008).

[44] D. Buskulic et al. (ALEPH Collaboration), Phys. Lett. B284, 177 (1992).

[45] P. Abreu et al. (DELPHI Collaboration), Phys. Lett. B332, 488 (1994).

[46] A. Abulencia et al. (CDF Collaboration), Phys. Rev. Lett. 97062003 (2006).

[47] S. Catani, Yu.L. Dokshitzer, M. Olsson,G. Turnock, B.R. Webber, Phys. Lett. B269 (1991) 432.

[48] " $B_{s}^{0}$ mixing in semileptonic $B_{s}^{0}$ decays using $D_{s}^{-} \rightarrow \phi \pi^{-}$decay mode", B. Abbott et al., D-Zero Note 4842 (2005).

[49] T. Sjöstrand, Comput. Phys. Commun. 82, 74 (1994).

[50] D.J. Lange, NIM A 462 (2001) 152; for details see http://www.slac.stanford.edu/ lange/EvtGen.

[51] " $B_{d}^{0}$ mixing measurement using Opposite-side Flavor Tagging", G. Borissov, S. Burdin, B. Casey, R. Jesik, D. Krop, Ph. Lewis, T. Moulik, A. Nomerotski, D. Tsybychev, D-Zero Note 4991 (2006).

[52] R. Akers et al., OPAL Collaboration, Z. Phys. C 66, 19 (1995).

[53] "Flavor Tagging Technique for $B_{s}^{0}$ Mesons", A. Rakitin, D-Zero Note 5210, v4.1 (2008).

[54] DELPHI Collab., Eur. Phys. J. C32 (2004), 185-208.

[55] http://www-d0.fnal.gov/ ${ }^{2}$ burdin/d0_private/d0note\&701/d0note4701dpluscontr.html

[56] V. M. Abazov et al., Phys. Rev. Lett. 99, 142001 (2007). 
[57] V. M. Abazov et al., Phys. Rev. Lett. 99182001 (2007).

[58] V. M. Abazov et al., Phys. Rev. Lett. 99, 052001 (2007).

[59] V. M. Abazov et al., Phys. Rev. Lett. 98, 121801 (2007).

[60] "Inclusive Single Muon Trigger Efficiency Study for RunIIa", S.W. Youn, B.C.K. Casey, D-Zero Note 5311 (2006).

[61] "Trigger Efficiency Study for RunII at D-Zero Using Inclusive Single Muon Sample", S.W. Youn, B.C.K. Casey, D. Buchholz, D. Tsybychev, D-Zero Note 5522 (2007).

[62] "The $K$ Factor in Semileptonic $B_{s}$ Decays using the $D_{s}^{-} \rightarrow \phi \pi^{-}$Decay Mode", J. Radigan, W. Taylor, D-Zero Note 5221 (2006).

[63] Private discussions with CDF and also with Ulrich Nierste.

[64] "Decay Length Resolution Studies: Application to $B_{s}^{0}$ Mixing and p17 Calibration", M.S. Anzelc, S. Burdin, D-Zero Note 5336 (2007).

[65] H.-G. Moser and A. Roussarie, NIM A 384 (1997) 491.

[66] S. Burdin et al., " $B_{s}^{0}$ Mixing in Semileptonic $B_{s}^{0}$ Decays Using the $D_{s}^{-}$to $\phi \pi$ Decay Mode and Unbinned Fit", D-Zero Note 5017 v8, http://www-d0.fnal.gov/Run2Physics/d0_private/eb/Run2EB_017/ 07/d0note5017_v8.pdf

[67] G. Borissov, S. Burdin, A. Nomerotski, "Measurement of Lifetime Ratio for Charged and Neutral B Mesons", D-Zero Note 4280 (2003).

[68] W. Taylor, "K Factor Correction for $B_{s}^{0} \rightarrow D_{s}^{-} \mu^{+} \nu X$ Decays", talk given at the August 25, 2005 B-Physics Meeting, http://www-d0.hef.kun.nl// askArchive.php?base $=$ agendaÉcateg $=a 05153163 i d=a 051531$ s1t2 $/$ transparencies

[69] V. M. Abazov et al., Phys. Rev. Lett. 94, 042001 (2005); V. M. Abazov et al., Phys. Rev. Lett. 98, 121801 (2007).

[70] G. Boix, D. Abbaneo, "The Bs oscillation amplitude analysis", JHEP 9908 (1999) 004; arXiv:hep-ex/9909033v1. 


\section{APPENDIX A}

\section{Branching Rates}

The following branching rates were used in the analysis. PDG values [6] were used when available, and EvtGen [50 inputs were used when the branching fraction was not listed in the PDG; the origin of the branching fraction is given as well.

- $\operatorname{Br}\left(B_{s}^{0} \rightarrow \mu^{+} \nu D_{s}^{-} X\right)=(7.9 \pm 2.4) \%$, where the total semileptonic Br was taken from the PDG, and fractions of exclusive channels were taken from EvtGen;

$$
\begin{aligned}
& -\operatorname{Br}\left(B_{s}^{0} \rightarrow \mu^{+} \nu D_{s}^{-}\right)=2.0 \% \\
& -\operatorname{Br}\left(B_{s}^{0} \rightarrow \mu^{+} \nu D_{s}^{-*}\right)=5.3 \% \\
& -\operatorname{Br}\left(B_{s}^{0} \rightarrow \mu^{+} \nu D_{s 0}^{*-}\right)=0.19 \% \\
& -\operatorname{Br}\left(B_{s}^{0} \rightarrow \mu^{+} \nu D_{s 1}^{\prime}\right)=0.35 \%
\end{aligned}
$$

- $\operatorname{Br}\left(B_{s}^{0} \rightarrow \tau^{+} \nu D_{s}^{-} X\right)=2.9 \%$, from Evtgen;

- $\operatorname{Br}\left(\tau^{+} \rightarrow \mu \nu \nu\right)=(17.36 \pm 0.06) \%$, from PDG;

- $\operatorname{Br}\left(B_{s}^{0} \rightarrow D_{s}^{+} D_{s}^{-} X\right)=4.6 \pm 2.2 \%$, from PDG;

- $\operatorname{Br}\left(B_{s}^{0} \rightarrow D D_{s}^{-} X\right)=8 \%$, from EvtGen;

- $\operatorname{Br}\left(\bar{B}_{s}^{0} \rightarrow D D_{s}^{-} X\right)=7.4 \%$, from EvtGen;

- $\operatorname{Br}\left(B^{+} \rightarrow D D_{s}^{-} X\right)=10.5 \pm 2.6 \%$, from PDG;

- $\operatorname{Br}\left(B^{0} \rightarrow D D_{s}^{-} X\right)=10.5 \pm 2.6 \%$, from PDG;

- $\operatorname{Br}\left(D_{s}^{-*} \rightarrow D_{s}^{-} X\right)=100 \%$

- $\operatorname{Br}\left(D_{s 0}^{*-} \rightarrow D_{s}^{-} X\right)=100 \%$ 
- $\operatorname{Br}\left(D_{s 1}^{\prime-} \rightarrow D_{s}^{-} X\right)=100 \%$

- $\operatorname{Br}\left(D_{s}^{-} \rightarrow \mu \nu X\right)=(6.3 \pm 0.8) \%$, from PDG, assuming the same partial width as for $D^{0}$ and $D^{+}$;

- $\operatorname{Br}\left(D^{0} \rightarrow \mu \nu X\right)=(6.5 \pm 0.8) \%$, from PDG;

- $\operatorname{Br}\left(D^{+} \rightarrow \mu \nu X\right)=(17.2 \pm 1.9) \%$, from PDG;

- $\operatorname{Br}\left(\bar{b} \rightarrow B^{0}\right)=39.7 \pm 1.0 \%$, from PDG;

- $\operatorname{Br}\left(\bar{b} \rightarrow B^{+}\right)=39.7 \pm 1.0 \%$, from PDG;

- $\operatorname{Br}\left(\bar{b} \rightarrow B_{s}^{0}\right)=10.7 \pm 1.1 \%$, from PDG; 


\section{APPENDIX B}

\section{VPDL $p d f$ Plots}

The figures displayed in this Appendix are additional plots of the VPDL $p d f$ s for RunIIa and RunIIb, to better display the differences in the distributions. Figure B.1 shows the signal and background distributions superimposed, for RunIIa (left) and RunIIb (right). Figure B.2 shows the VPDL $p d f$ for RunIIa, with and without the event-by-event SFs applied; the same for RunIIb is shown in Figure B.3.

RunIIa and and RunIIb $p d f$ s without the event-by-event $S F$ s are shown in Figures B.4 and B.5 respectively, for comparison to Figures B.2 and B.3 or Figures 3.20 and 3.21.
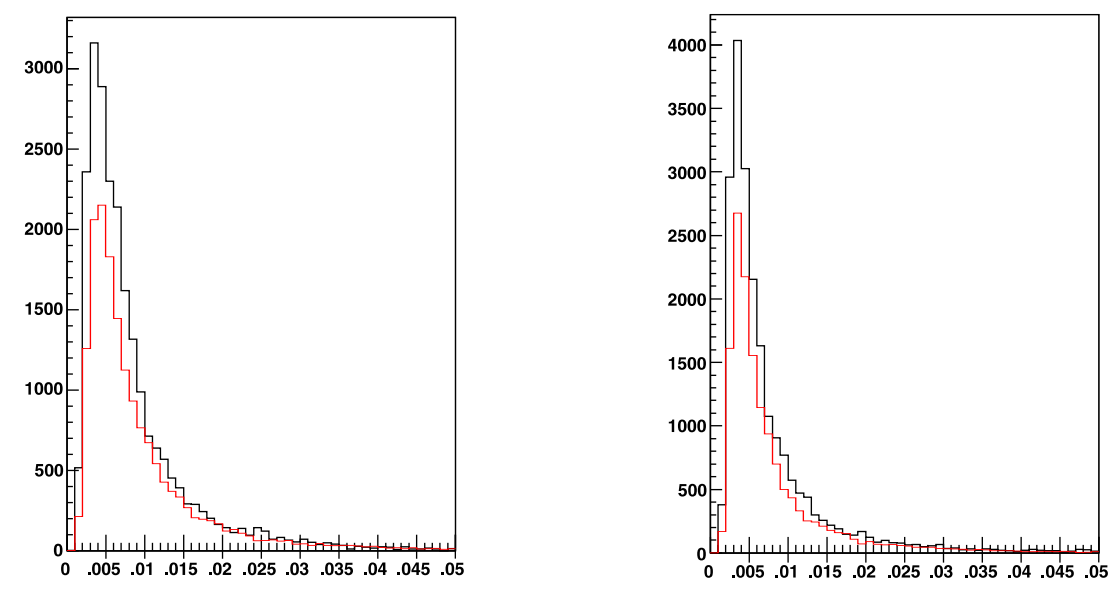

Figure B.1. Distributions of VPDL errors for signal (black) and combinatorial background (red) in RunIIb data. 

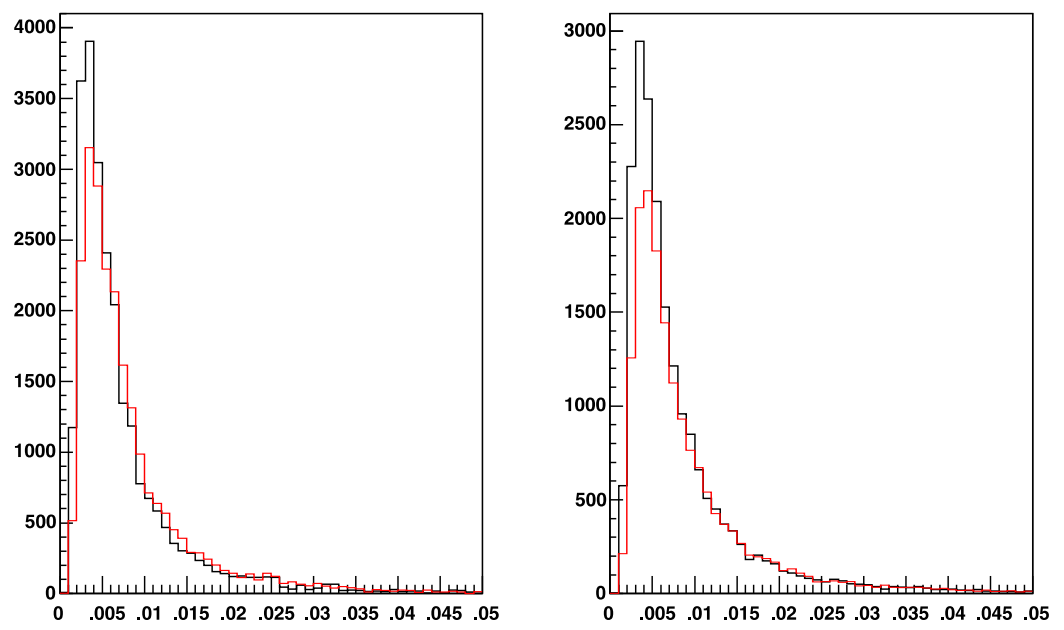

Figure B.2. Distributions of VPDL errors for signal (left) and combinatorial background (right) in RunIIa data. The distributions without the event-by-event $S F$ s applied are shown in black, while those with the eventby-event $S F$ s applied are shown in red.
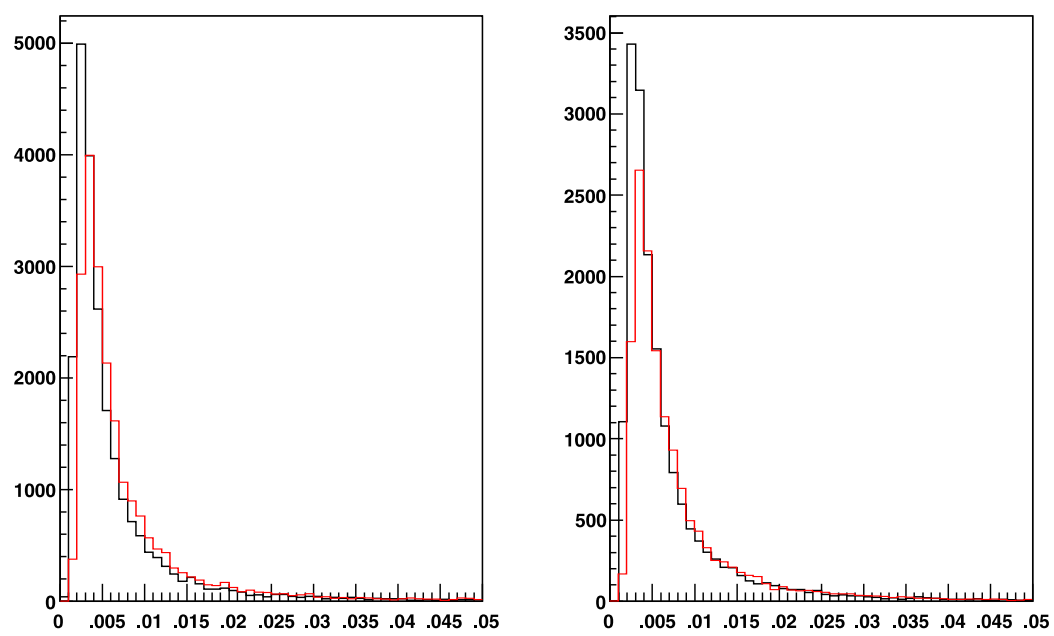

Figure B.3. Distributions of VPDL errors for signal (left) and combinatorial background (right) in RunIIb data. The distributions without the event-by-event $S F$ s applied are shown in black, while those with the eventby-event $S F$ s applied are shown in red. 

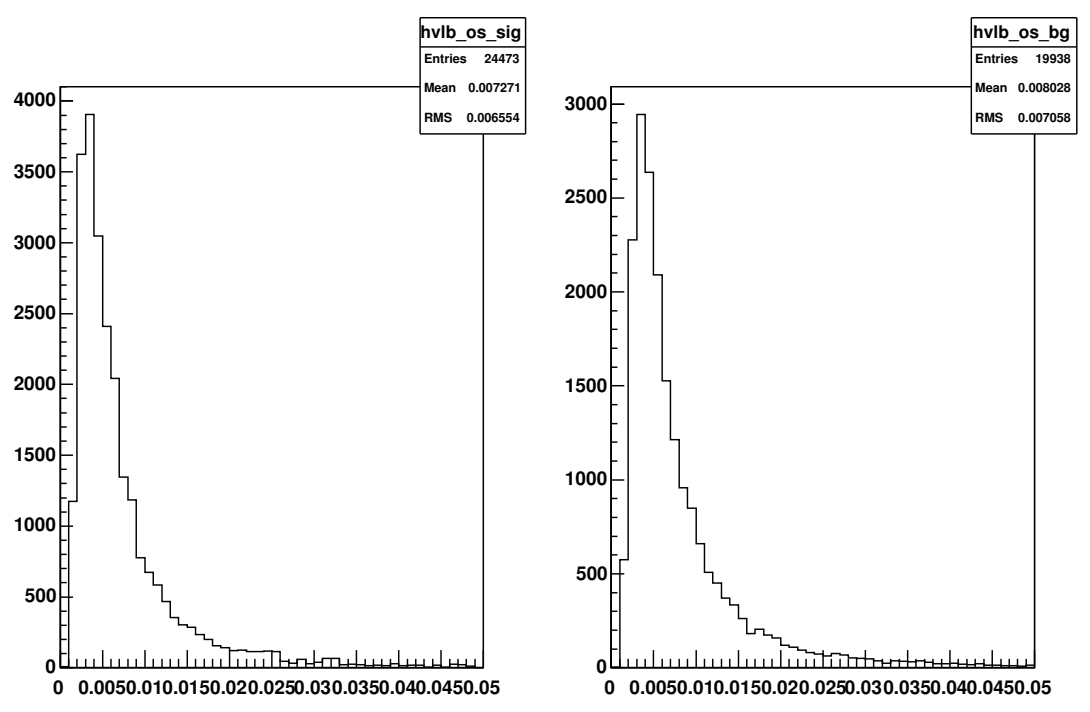

Figure B.4. Distributions of VPDL errors for signal (left) and combinatorial background (right) in RunIIa data, without the event-by-event $S F$ s applied.
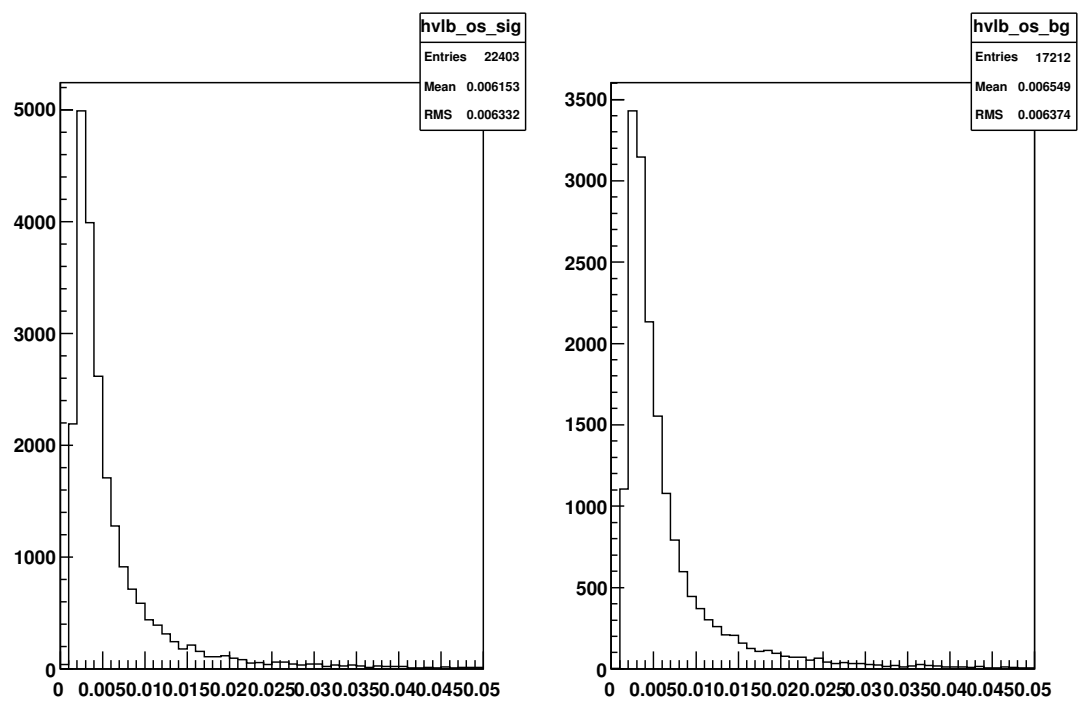

Figure B.5. Distributions of VPDL errors for signal (left) and combinatorial background (right) in RunIIb data, without the event-by-event $S F_{\mathrm{S}}$ applied. 


\section{APPENDIX C}

\section{List of IP-Biased Triggers in RunIIa and RunIIb Data}

The following are the lists of impact parameter biasing triggers for RunIIa and RunIIb data. Note that these are complete lists for the entirety of the RunIIa data collection period and for the RunIIb period up to the August 2007 shutdown.

\section{C.1. RunIIa}

The RunIIa IP-biased triggers are divided into three categories:

- Two-Track Triggers, which required two tracks in the $\phi$ mass and which must have had significant impact parameters with respect to the PV,

- Muon Triggers, that required a muon in the event to have a significant impact parameter, and

- Random Triggers, which required one or more random tracks with significant impact parameter.

\section{C.1.1. Two-Track Triggers}

$\begin{array}{cc}\text { ML1_TMM_IPPHI } & \text { MM1_TMM_IPPHI } \text { ML2_MM_IPPHI } \\ \text { MM1_HI_TMM_IPPHI } & \text { MEB1_MM_IPPHI }\end{array}$




\title{
C.1.2. Muon Triggers
}

\author{
ML1_IPTMM_IMP_V MM1_IPTMM5_IMPV ML1_IPTLM_IMP_V \\ MM1_IPTLM5_IMPV MM1_HI_IPTMM
}

\section{C.1.3. Random Triggers}

$\begin{array}{ccc}\text { ML1_TMM_2IP_IMPV } & \text { ML1_TMM_3IP_IMPV } & \text { ML1_TMM_4IP_IMPV } \\ \text { MM1_TMM_IMP_2IPV } & \text { MM1_TMM_IMP_3IPV } & \text { MM1_TMM_IMP_4IPV } \\ \text { ML1_TLM_2IP_IMPV } & \text { ML1_TLM_3IP_IMPV } & \text { ML1_TLM_4IP_IMPV } \\ \text { MM1_TLM_IMP_2IPV } & \text { MM1_TLM_IMP_3IPV } & \text { MM1_TLM_IMP_4IPV } \\ \text { ML2_2IPMM_IMP_V } & \text { ML2_3IPMM_IMP_V } & \text { ML2_4IPMM_IMP_V } \\ \text { ML3_2IPMM_IMP_V } & \text { MM1_HI_TMM_2IPV } & \text { MM1_HI_TMM_3IPV } \\ \text { MEB1_2IPMM_IMP_V } & \text { MEB1_3IPMM_IMP_V } & \text { MEB1_4IPMM_IMP_V } \\ \text { MUJB_MM0_BID } & \text { JT1_ACO_MHT_BDV } & \text { JT2_3JT15L_IP_VX } \\ \text { JT3_3JT10L_LM3_V } & \text { JT7_3JT15L_IP_VX } & \text { MUJ1_2JT12_LMB_V } \\ \text { MUJ1_JTHATK_LMVB } & \text { MUJ2_2JT12_LMB_V } & \text { MUJ2_JTHATK_LMVB } \\ \text { ZBB_TLM3_2JBID_V } & \text { ZBB_JT_HATKTLMV } & \text { EZBB_SHT122J12VB } \\ \text { MT3_L2M0_MM3_IP } & \text { ZB1_TLM3_2JBID_V } & \text { ZB1_JT15HA_TLM8V } \\ \text { E3_SHT122J12VB } & & \end{array}$

\section{C.2. RunIIb}

The RunIIb IP-biasing triggers have not been broken down into different categories. The full list of IP-biased triggers follows. 


\begin{tabular}{|c|c|c|}
\hline M3_IPTMM_IMP_V & M3_TMM_2IP_IMPV & M3_TMM_3IP_IMPV \\
\hline M3_TMM_4IP_IMPV & M3_TMM_IPPH & M3_2IPMM_IMP_V \\
\hline M3_3IPMM_IMP_V & M3_4IPMM_IMP_V & M3_MM_IPPHI \\
\hline M4_IPTMM_IMP_V & M4_TMM_2IP_IMPV & M4_TMM_3IP_IMPV \\
\hline M4_TMM_4IP_IMPV & M4_TMM_IPPH & M4_2IPMM_IMP_V \\
\hline M4_3IPMM_IMP_V & M4_4IPMM_IMP_V & M4_MM_IPPHI \\
\hline M5_TMM_IMP_2IPV & M5_TMM_IMP_3IPV & M5_TMM_IMP_4IPV \\
\hline M5_IPTMM5_IMPV & M5_TMM_IPPHI & M5_IPTMM5_IMPH \\
\hline M5_TMM_IMP_2IPH & M6_TMM_IMP_2IPV & M6_TMM_IMP_3IPV \\
\hline M6_TMM_IMP_4IPV & M6_IPTMM5_IMPV & M6_TMM_IPPHI \\
\hline M6_TMM_IMP_2IPH & M6_IPTMM5_IMPH & ME6_IPTMM_IMP_V \\
\hline ME6_TMM_2IP_IMPV & ME6_TMM_3IP_IMPV & ME6_TMM_4IP_IMPV \\
\hline ME6_TMM_IPPHI & ME6_2IPMM_IMP_V & ME6_3IPMM_IMP_V \\
\hline ME6_4IPMM_IMP_V & ME6_MM_IPPHI & M5_TLM3_2JBID_V \\
\hline M5_JT_HATKTLMVB & M6_TLM3_2JBID_V & M6_JT_HATKTLMVB \\
\hline MUJ1_2JT12_LMB_V & MUJ1_JTHATK_LMVB & MUJ2_2JT12_LMB_V \\
\hline MUJ2_JTHATK_LMVB & MUJ3_2JT12_LMB_V & MUJ3_JTHATK_LMVB \\
\hline MUJ4_2JT12_LMB_V & MUJ4_JTHATK_LMVB & MUJ5_MM0_BID \\
\hline MJ_ACO_MHT_BDV & JT1_ACO_MHT_BDV & JT2_ACO_MHT_BDV \\
\hline JT2_3JT10L_LM3_V & JT2_3JT15L_IP_VX & JT3_ACO_MHT_BDV \\
\hline JT3_3JT10L_LM3_V & TT3_3JT15L_IP_VX & \\
\hline
\end{tabular}

